\title{
29581
}

\section{ELECTRICAL IMPEDANCE TOMOGRAPHY USING INDUCED CURRENTS}

\author{
A Ph. D. Thesis \\ Presented by \\ NEVZAT GÜNERI GENÇER
}

to

the Graduate School of Natural and Applied Sciences of Middle East Technical University in Partial Fulfillment for the Degree of

DOCTOR OF PHILOSOPHY

in

ELECTRICAL AND ELECTRONICS ENGINEERING

$\infty-$

MIDDLE EAST TECHNICAL UNIVERSITY

ANKARA

July, 1993 
Approval of the Graduate School of Natural and Applied Sciences.

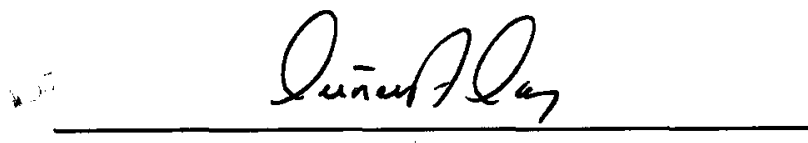

Prof. Dr. IsmailTosun

Director

I certify that this thesis satisfies all the requirements as a thesis for the degree of Doctor of Philosophy.

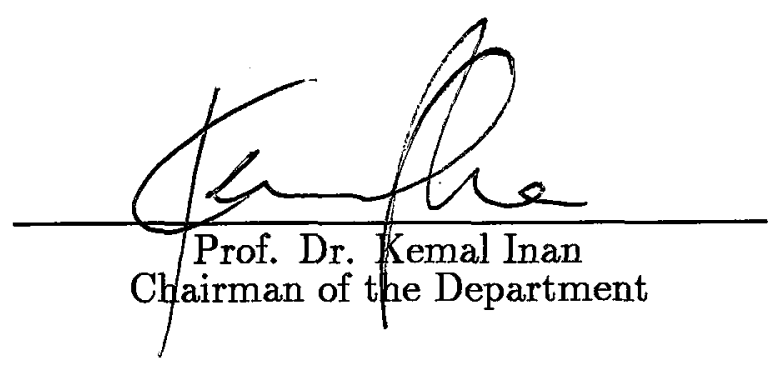

We certify that we have read this thesis and that in our opinion it is fully adequate, in scope and quality, as a thesis for the degree of Doctor of Philosophy in Electrical and Electronics Engineering.

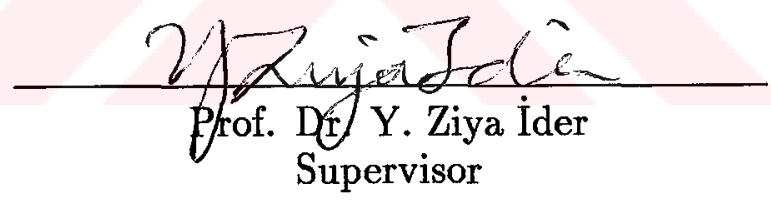

Examining Committee in Charge:

Prof. Dr. Mete Severcan(Chairman) letflevenan

Prof. Dr. Y. Ziya İder

Prof. Dr. Hayrettin Köymen

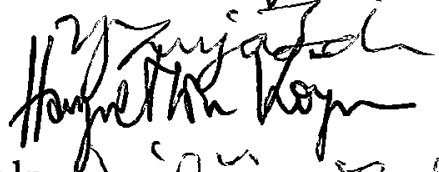

Assoc. Prof. Dr. Nilgün Günalp

Assoc. Prof. Dr. Mustafa Kuzuoğlu

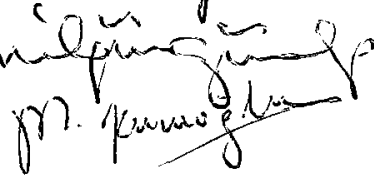




\section{ABSTRACT \\ ELECTRICAL IMPEDANCE TOMOGRAPHY USING INDUCED CURRENTS \\ GENÇER, NEVZAT GÜNERI}

Ph. D. in Electrical and Electronics Engineering

Supervisor: Prof. Dr. Y. Ziya IDER

July, 1993, 107 pages.

The mathematical basis of a new imaging modality, Induced Current Electrical Impedance Tomography (EIT), is investigated. The ultimate aim of this technique is the reconstruction of conductivity distribution of the human body, from voltage measurements made between electrodes placed on the surface, when currents are induced inside the body by applied time varying magnetic fields. In this study the two-dimensional problem is analyzed. A specific 9 coil system (with circular coils) for generating 9 different exciting magnetic fields $(50 \mathrm{kHz})$ and 16 measurement electrodes around the object are assumed. The partial differential equation for the scalar potential function in the conductive medium is derived and Finite Element Method is used for its solution. A linear system of equations is derived which relates the perturbation in measurements to the conductivity perturbations. Singular Value Decomposition of the coefficient matrix (sensitivity matrix) shows that, for this particular coil configuration, there are $135(9 \times 15)$ independent measurements. It is found that measurements are less sensitive to changes in conductivity of the object's interior. While in this respect induced current EIT is slightly inferior to the technique of injected current EIT (using Sheffield protocol), its sensitivity matrix is better conditioned. The images obtained by simulated data are found to be comparable to injected current EIT images in resolution. An alternative coil configuration is suggested to improve the performance of induced current EIT and simulation studies are performed. The feasiblity of this new imaging modality, induced current EIT, 
is investigated by realizing a PC based data acquisition system. Real data sets are collected from the boundary of a saline filled two dimensional phantom (inhomogeneity is represented by a delrin disk) using 6 circular coils for current induction (i.e., 90 independent measurements). It is shown that conventional data acquisition hardware of EIT can be used for data collection in induced current EIT, by addition of a coil drive electronics. The measured boundary potential gradients are shown to be accurate enough to provide good estimates of actual object distributions.

Keywords : Electrical Impedance Tomography, Applied Potential Tomography, Impedance Imaging, Finite Element Method.

Science Code : 609.01 .04 


\author{
ÖZ \\ INDÜKLENEN AKIMLAR ILE \\ ELEKTRIKSEL EMPEDANS TOMOGRAFISI
}

GENÇER, NEVZAT GÜNERI

Doktora Tezi, Elektrik ve Elektronik Mühendislig̃i Anabilim Dalı

Tez Yöneticisi: Prof. Dr. Y. Ziya IDER

Temmuz, 1993, 107 sayfa.

Akım uygulamalı Elektriksel Empedans Tomografisi (EET) adı ver ilen yeni bir görüntüleme yönteminin matematiksel temelleri araştırılmıştır. Bu yöntemin ana amacı, insan vücudundaki doku iletkenlik dağılımının görüntülenmesidir. Bu amaçla, vücut üzerine zamanla değişen manyetik alanlar uygulanarak akım indüklenmekte ve vücut yüzeyine yerleștirilen elektrotlardan voltaj farkı ölçümleri elde edilmektedir.

Bu çalı̧̧mada, iki-boyutlu problem analiz edilmiştir. Uzayda dağılımı farklı 9 değişik manyetik alanın $(50 \mathrm{KHz})$ uygulandiğı ve vücut yüzeyine 16 elektrodun yerleştirildiği varsayılmıştir. İletken ortamda skalar potansiyel fonksiyonun sagladığı kısmi difransiyel denklem türetilmis ve çözümü için Sonlu Elemanlar Yöntemi kullanılmıştır. İletkenlik dağılımındaki değişimi ölçüm değişimlerine bağlıyan bir doğrusal denklem takımı elde edilmiştir. Öz Değer Ayrıştırma (Singular Value Decomposition) tekniğinin katsayı matrisi (duyarlılık matrisi) üzerine uygulanması ile bu özel sarım yapısı için 135 $(9 \times 15)$ bağımsız ölçme yapılabildiği gösterilmiştir. Akım indüklemeli EET kullanıldığında ölçümler iç bölgelerdeki iletkenlik değişimlerine daha az duyarlı olduğu halde, duyarlılık matrisi daha iyi durumlanmıştır (better conditioned). Bu yöntem ile çözüm verileri kullanılarak akım uygulamalı EET ile ayn kalitede görüntüler elde edilebileceği gösterilmiştir. Akım indüklemeli EET'nin performansının arttırılması icin alternatif bir sarım yapısı önerilmiş ve özellikleri 
incelenmiştir.

Akım indüklemeli EET'nin uygulanabilirliğinin anlaşılabilmesi için PC ile kontrol edilen bir prototip veri toplama sistemi gerçekleştirilmiştir. 6 dairesel sarımlı bir uyarım sistemi ile, dairesel bir kabın yüzeyinden voltaj farkı verileri elde edilmiştir (90 bağımsız ölçüm). Kullanılan kabın içine iletkenliği tuz ile ayarlanan saf su doldurulmuş ve farklı iletkenlik dağılımları delrin disklerin su içerisinde değişik yerlere konması ile sağlanmıştır. Bilinen EET veri toplama sistemlerinin bir sarım sürme elektroniği eklenerek akım indüklemeli EET için de kullanılabileceği gösterilmiştir. Toplanan gerçek verilerle gerçek iletkenlik dağ̊lımlarına yakın iletkenlik dağılımı görüntüleri elde edilmiştir.

Anahtar Kelimeler : Elektriksel Empedans Tomografisi, Potansiyel Uygulamalı Tomografi, Empedans Görüntülemesi, Sonlu Elemanlar Yöntemi

Bilim Dalı Sayısal Kodu : 609.01.04 


\section{ACINOWLEDGENENTS}

I am greatly indebted to my supervisor Prof. Dr. Y. Ziya İder for his invaluable guidance and encouragement throughout my graduate studies. His significant contributions in every stage of this research are gratefully acknowledged. I wish to express my graditude to Assoc. Prof. Dr. Mustafa Kuzuoğlu for his continuous support, encouragement and suggestions throughout 1.]jis study. Thanks are due to Prof. Dr. Hayrettin Köymen for his various advices and encouragement for my studies in METU.

I am grateful to Assoc. Prof. Dr. Nilgün Gïnalp and Ass. Prof. Dr. Liemal Lebleljicioğlu for their valuable criticisms and suggestions. Thanks are due to Ass. Prof. Dr. B. Murat. Eyuloğlu for many stimulating discussions using Internet.

I wish to thank to Haldun Özdemir for his valuable comments and efforts during the realization of the data acquisition system and Vasfi Ergun for his continuous support on computer problems.

Special thanks are due to Aykut Bultan, Serhat Özyar, Almet Baykal and Ergin Atalar for they have been a source of good-humour, help and advice throughout my studies. I would also like to thank to my dear friends Ender Turan, Bora Nakiloğlu, Cem Tarakcı, Cem Sakı, Tugan Müftüler, Uğur Baysal, Tolga Ciloğlu, Hüseyin Yavuz, Y. Cağatay Tekmen and Ugur Laloğlu for their supports in every stage of this thesis. Thanks are due to Safure Bulut for her friendship and helps about the format of this thesis. Special thanks are due to my girl friend Handan Can for her patience and support during thesis studies.

Finally, I wish to express my deep gratitude to my family. I owe them a lot. 
TABLE OF CONTENTS

Page

ABSTRACT $\ldots \ldots \ldots \ldots \ldots \ldots \ldots \ldots \ldots \ldots \ldots \ldots \ldots \ldots \ldots \ldots \ldots \ldots \ldots$, iii

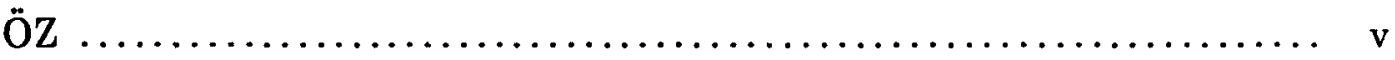

ACKNOWLEDGEMENTS $\ldots \ldots \ldots \ldots \ldots \ldots \ldots \ldots \ldots \ldots \ldots \ldots \ldots \ldots \ldots \ldots \ldots$ vii

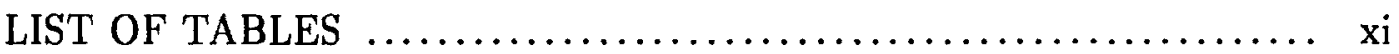

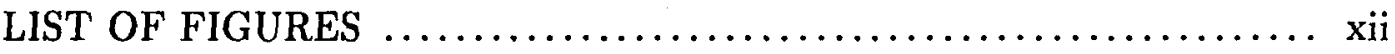

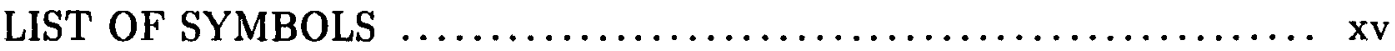

CHAPTER I : INTRODUCTION ........................... 1

1.1 Medical Imaging . . . . . . . . . . . . . . 1

1.2 Electrical Impedance Tomography (EIT) $\ldots \ldots \ldots \ldots \ldots$

1.3 Historical development of EIT . . . . . . . . . 5

1.4 EIT in Middle East Technical University . . . . . . . 11

1.5 EIT Using Induced Currents . . . . . . . . . . . . . . 12

1.6 The Objectives of this thesis $\ldots \ldots \ldots \ldots \ldots \ldots$

1.7 Structure of this Thesis $\ldots \ldots \ldots \ldots \ldots \ldots \ldots$

CHAPTER II : FORMULATION OF THE FORWARD PROBLEM $\ldots . .16$

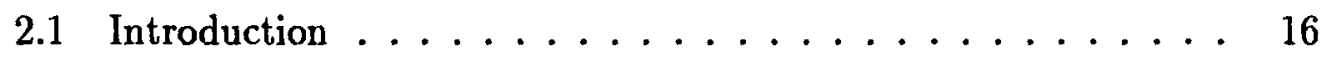

2.2 Basic Field Equations $\ldots \ldots \ldots \ldots \ldots \ldots$

2.3 Formulation of the Partial Differential Equations governed by Scalar and Magnetic Vector Potential Functions . . . . . 17

2.4 Relation of actual measurements to $\phi$ and $\vec{A} \ldots \ldots \ldots 19$

CHAPTER III : SOLUTION OF THE FORWARD PROBLEM $\ldots \ldots \ldots 22$

3.1 The Series Expansion Method (SEM) for solving $\phi \ldots \ldots \ldots .22$

3.1.1 2-D circular object with uniform conductivity . . . 22 
3.1.2 2-D circular object with a concentric inhomogeneity .

3.2 Formulation and Solution of the Forward Problem using Finite Element Method (FEM) . . . . . . . . . . . 25

3.3 Numerical Results . . . . . . . . . . . . . . . 28

3.3.1 Comparison of scalar potential solutions obtained by FEM and SEM . . . . . . . . . . . . 28

3.3.2 Calculation of induced current components by FEM . 29

3.3.3 Interpreting the sources of perturbations in $\phi \ldots . .33$ CHAPTER IV : THE INVERSE PROBLEM $\ldots \ldots \ldots \ldots \ldots \ldots \ldots \ldots \ldots$

4.1 Introduction . . . . . . . . . . . . 35

4.2 Properties of the Sensitivity matrix $R \ldots \ldots 36$

4.2.1 Singular Value Decomposition technique and general matrix properties ............. 36

4.2.2 Effects of coil configuration on $\kappa(R) \ldots \ldots . \ldots 37$

4.2.3 Number of independent measurements for an $N$-electrode system ..................... 38

4.2 .4 Sensitivity analysis ............. 43

4.3 Image Reconstruction . . . . . . . . . . . . . . 44

4.4 An alternative coil configuration ............ 48

CHAPTER V : DESIGN OF A DATA ACQUSITION SYSTEM $\ldots \ldots \ldots 53$

5.1 Introduction .................. 53

5.2 System Overview ...................... 54

5.3 Circuit Descriptions ................... 54

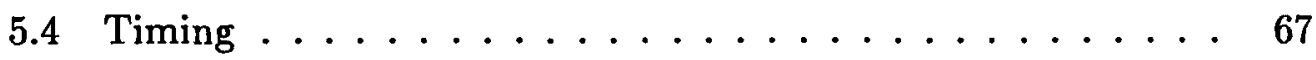

5.5 Experimental Results ................. 68

5.5.1 Data Comparison .............. 68

5.5.2 Image Reconstruction Using Real Data . . . . . . 71

CHAPTER VI : CONCLUSION AND DISCUSSION ............ 83

6.1 Summary of this Work ............... 83

6.2 Conclusions reached ................ 83 
APPENDICES

APPENDIX A. CALCULATION OF $\vec{A} \ldots \ldots \ldots \ldots \ldots \ldots \ldots \ldots \ldots . \ldots . \ldots . \ldots$

APPENDIX B. FEM FORMULATION $\ldots \ldots \ldots \ldots \ldots \ldots \ldots \ldots \ldots \ldots$

APPENDIX C : VALIDATION OF THE ASSUMPTIONS $\ldots \ldots \ldots \ldots . . .99$

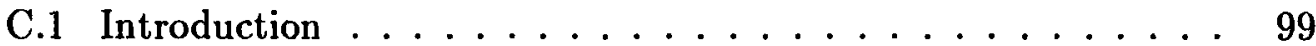

C.2 Validation of the First Assumption . . . . . . . . . . 100

C.3 Validation of the Second Assumption . . . . . . . . . . . . 101

C.4 Results . . . . . . . . . . . . . . . . . . . . 105

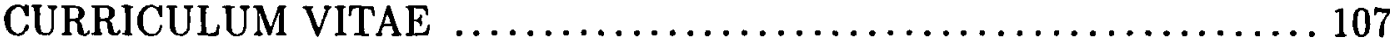




\section{LIST OF TABLES}

Table 1.1 Typical values of resistivity and X-ray linear attenuation coefficient of five biological tissues. (From Barber et al (1983)

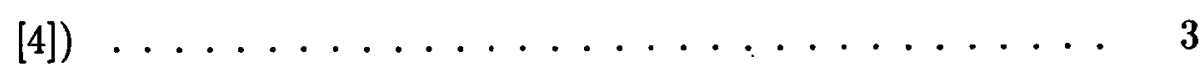

Table 3.1 Comparison of Boundary Potential differences calculated by SEM and FEM for both uniform and non-uniform object distributions. ..................... 30

Table 5.1 Comparison of the calculated and measured boundary potential differences. . . . . . . . . . . . . . . 70

Table 5.2 Collected data for the first object distribution. . . . . . . 74

Table 5.3 Collected data for the second object distribution $\ldots \ldots \ldots 75$

Table 5.4 Collected data for the third object distribution $\ldots \ldots 76$

Table 5.5 Collected data for the fourth object distribution $\ldots \ldots$. . 77

Table 5.6 Truncation level singular values and number of terms included in image reconstruction due to assigned six different SNR .

Table C.1 Comparison of the magnitudes of the secondary and primary magnetic vector potentials . . . . . . . . . . 102

Table C.2 Comparison of boundary potential differences obtained by SEM formulations with and without displacement currents . . . 106 


\section{LIST OF FIGURES}

Figure 1.1 General principles of excitation and data collection in injected current EIT. . . . . . . . . . . . . . . 4

Figure 1.2 General principles of excitation and data collection in induced current EIT. . . . . . . . . . . . . . . 13

Figure 3.1 A uniform circular object placed in a circular coil . . . . . 23

Figure 3.2 A circular object with concentric inhomogeneity placed in a circular coil . . . . . . . . . . . . . . . 24

Figure 3.3. The 541-node, 1016-element mesh for FEM formulations . . . 27

Figure 3.4 Plot of current density distribution and its components for the first coil configuration. . . . . . . . . . . . 31

Figure 3.5 Plot of current density distribution and its components for the second coil configuration. . . . . . . . . . . 32

Figure 3.6 $\Delta(\nabla \phi)$ field when a uniform conductivity distribution is perturbed on small rectangular region(s) . . . . . . . 34

Figure 4.1 Placement of 3 circular coils on the plane of a circular object. $\quad 37$

Figure 4.2 Singular value patterns of sensitivity matrices obtained for the 3-coil system due to different coil center shifts $\left(R_{s}\right) . \ldots 39$ 
Figure 4.3 Singular value patterns of sensitivity matrices obtained for the 3-coil system due to changes in the radius $\left(R_{s}\right)$ of coils. . . 40

Figure 4.4 Singular value patterns of induced and injected current EIT .

Figure 4.5 Sensitivity of four measurements to conductivity perturbations 45

Figure 4.6 Display of total lead sensitivity vectors of induced and injected current EIT . . . . . . . . . . . . . . . 46

Figure 4.7 Average sensitivity values of injected and induced current EIT systems evaluated on a radial line . . . . . . . . . 47

Figure 4.8 Comparison of reconstructed images obtained by induced and injected current EIT . . . . . . . . . . . . . . 49

Figure 4.9 An alternative coil configuration $\ldots \ldots \ldots . \ldots 50$

Figure 4.10 Induced current density distribution and its components when alternative coil configuration is used .......... 51

Figure 5.1 Block diagram of the prototype . . . . . . . . . 55

Figure 5.2 Circuit diagram of the power amplifier . . . . . . . . 56

Figure 5.3 Circuit diagrams of (a) the overall current drive multiplexing circuit, (b) relay drive circuit . . . . . . . . . . 58

Figure 5.4 Measurement apparatus . . . . . . . . . . . 60

Figure 5.5 Circuit diagram of the voltage difference measurement system. 61

Figure 5.6 Circuit diagram of the opto-isolation circuit . . . . . . . 66 
Figure 5.7 Coil configuration and object distribution for the second ex-

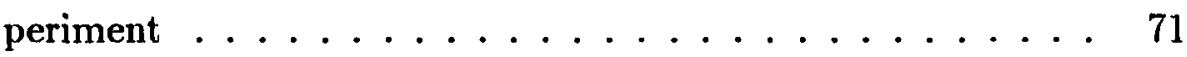

Figure 5.8 Four different object distributions obtained by placing the delrin disk at different locations inside the saline solution . . . . 72

Figure 5.9 Coil placement strategy . . . . . . . . . . . . 73

Figure 5.10 Recontructed images of the first object distribution assuming different values for SNR . . . . . . . . . . 78

Figure 5.11 Recontructed images of the second object distribution assuming different values for SNR . . . . . . . . . . 79

Figure 5.12 Recontructed images of the third object distribution assuming different values for SNR . . . . . . . . . . 80

Figure 5.13 Recontructed images of the fourth object distribution assuming different values for SNR . . . . . . . . . 81

Figure B.1 A mesh with 4 nodes and 2 triangular elements . . . . . 98

Figure C.1 The object distribution used for the solution of the scalar potential function including the effects of displacement currents 


\section{LIST OF SYMBOLS}

$\vec{E}$ : electric field intensity $($ Volt $/ m)$

$\vec{D}$ : electric displacement (coulombs $/ m^{2}$ )

$\vec{B}$ : magnetic field density (Weber $/ \mathrm{m}^{2}$ )

$\vec{J}$ : conduction current density (Ampere $/ \mathrm{m}^{2}$ )

$\rho:$ charge density $\left(\right.$ coulomb $\left./ m^{3}\right)$

$\sigma:$ electrical conductivity (Ampere $/$ Volt $\cdot m$ or Siemens $/ m$ )

$\epsilon:$ permittivity $(\mathrm{farad} / \mathrm{m})$

$\mu_{0}:$ permeability of free space (Henry $/ m$ )

$\omega:$ radial frequency $(\mathrm{radian} / \mathrm{sec})$

$\vec{A}$ : magnetic vector potential

$\vec{n}$ : outward unit normal vector

$\Omega$ : object region

$\partial \Omega$ : object boundary

$a, b:$ points on the object boundary $\partial \Omega$

$v_{a b}:$ voltage difference between points $a$ and $b$ on $\partial \Omega$

$\sigma_{0}:$ initially assumed conductivity distribution

$\phi_{0}$ : scalar potential function corresponding to $\sigma_{0}$

$A_{n}$ : normal component of magnetic vector potential $\vec{A}$

$r, \theta:$ polar coordinate variables 
$m, i, j:$ indexes

$M:$ number of different exciting fields

$R_{1}$ : radius of concentric inhomogeneity

$R, R_{2}:$ circular object radius in different problems

$R_{c}:$ circular coil radius

$R_{s}:$ coil center shift with respect to object origin

$g_{k}$ : difference of scalar potentials between $(k+1)^{t h}$ and $k^{\text {th }}$ electrodes

$a_{m}, b_{m}, c_{m}, d_{m}, e_{m}, f_{m}:$ series expansion coefficients of $m^{\text {th }}$ term

$a_{m R}, b_{m R}, c_{m R}, d_{m R}, e_{m R}, f_{m R}$ :real parts of series expansion coefficients of $m^{\text {th }}$ term

$a_{m I}, b_{m I}, c_{m I}, d_{m I}, e_{m I}, f_{m I}:$ imaginary parts of series expansion coefficients of $m^{\text {th }}$ term

$\sigma_{1}, \sigma_{2}$ : conductivities of concentric circular inhomogeneity and background conductivity, respectively

$\alpha$ : conductivity contrast (i.e., $\sigma_{1} / \sigma_{2}$ )

$\beta$ : ratio of concentric inhomogeneity radius to circular object radius (i.e., $\left.R_{1} / R_{2}\right)$

$C(m), D(m), E(m), F(m)$ : coefficients related to angular spatial frequency content of $A_{n}$

$S:$ coefficient matrix derived using FEM

$\bar{\sigma}:$ vector of element conductivities

$\bar{\sigma}_{0}:$ vector of initially assumed conductivity of elements

$\Delta \bar{\sigma}:$ vector of perturbations in element conductivities

$\bar{V}:$ vector of scalar potentials at the nodes of FEM mesh

$\bar{V}_{0}$ : vector of scalar potentials at the nodes of FEM mesh corresponding to $\overline{\sigma_{0}}$ 
$\bar{g}:$ vector of surface scalar potential differences for a certain coil configuration

$C$ transformation matrix relating $\bar{g}$ to $\bar{V}$

$\Delta \bar{g}:$ vector representing the perturbation in $\bar{g}$

$\Delta \bar{G}:$ vector of perturbation in surface scalr potential differences for all coil configurations

$R$ : sensitivity matrix

$A$ : an arbitrary $m \times n$ matrix $(m<n)$

$\bar{u}_{i}: i^{\text {th }}$ right singular vector of $A$

$\bar{v}_{i}: i^{\text {th }}$ left singular vector of $A$

$U$ : decomposition matrix including right singular vectors of $A$

$V$ : decomposition matrix including left singular vectors of $A$

$\Sigma$ : diagonal matrix with diagonal entries equal to singular values of $A$

$\lambda_{i}: i^{\text {th }}$ singular value of $A$

$\kappa(\cdot)$ : condition number of a matrix

$\bar{T}:$ total lead sensitivity vector 


\section{CHAPTER I}

\section{INTRODUCTION}

\subsection{Medical Imaging}

Medical Imaging is being developed as a tool for both diagnosis and treatment of illnesses in human body tissues. Several imaging modalities are being used to image different physical properties of tissues in human body. The common approach of these techniques is, application of some sort of energy on the object and measuring the response of object as a result of its interaction with the applied energy. The type of energy and measured quantity depends on the choice of physical property that is to be imaged.

In X-ray Computerized Tomography (X-ray CT), X-ray beams are send to a selected slice of a body using a proper scanning mechanism and transmitted energy is measured from the other side. Thereafter, these measurements are used to reconstruct the $\mathrm{X}$-ray attenuation coefficient distribution of the slice by using a proper image reconstruction algorithm [40], [49], [56], [78]

In Ultrasonic Reflection Imaging, ultrasonic pulses are propagated through the body. The reflected waves turning back from the interfaces on the path of the propagated beam are measured and complete tissue interface maps are reconstructed (B Scan Imaging) [56]. In Ultasonic Computed Tomography, the exact pressure of the wave on the far side of the object is recorded as a function of time. The attenuation observed on the pressure field and the delay in the signal induced by the object make it possible to reconstruct the attenuation coefficient and the refractive index distribution of the object [49].

In Magnetic Resonance Imaging, the object is first subjected to a 
strong uniform magnetic field. It is known that nuclei that contain odd number of protons or neutrons posses magnetic moments. When these magnetic moments are placed in external magnetic field they tend to align themselves parallel to the applied field. Actually a perturbed magnetic moment behaves like a gyroscope precessing around the direction of the field. The precession fequency (Larmor frequency) is determined by the magnitude of the local field out of a constant (gyrometric constant). A spatially varying radio frquency (RF) field at larmor frequency is used to code each position on the object slice with a unique resonance frequency. As a result of the RF pulse, the magnetic moments are perturbed from their original position. And when the excitation ceases, the rotating magnetic moments decay to their equilibrium state emitting signals at resonant frequencies. These signals are detected by the same coils that produce the RF pulse and are used for imaging, for example, the hydrogen density distribution in human body [49], [56].

In Emission Computed Tomography (ECT), the region under study becomes an active source because of radiactive material administration. The radiation continues until the material is elliminated by the body or decays. This makes ECT a functional imaging modality since during the decay time it is possible to follow the function of a selected organ. The selective location of this material depends on either the material property or the property of the chemical form it is bound in [56]. This technique is usually considered as two separate modalities, namely Single Photon Emission Computed Tomography (SPECT) and Positron Emission Tomography (PET) [78]. In SPECT, radioisotopes which emit a single $\gamma$-ray per nuclear disintegration are used. In PET, the nuclear disintegration of some other radioisotopes produces positrons whose annihilation in tissue results in emission of two $\gamma$-rays of the same energy.

The reason of the development of different imaging modalities mainly based on the need of the complementary information they can provide to the existing imaging systems. 
Table 1.1. Typical values of resistivity and X-ray linear attenuation coefficient of five biological tissues. (From Barber et al (1983) [4])

\begin{tabular}{||c|c|c||}
\hline Tissue & $\begin{array}{c}\text { Resistivity } \\
\text { ohm } / m\end{array}$ & $\begin{array}{c}\text { X-ray attenuation } \\
\text { coefficient } \\
m^{-1}\end{array}$ \\
\hline Bone & 150 & 35.0 \\
Muscle & 3.0 & 20.4 \\
Blood & 1.6 & 20.4 \\
Fat & 15.0 & 18.5 \\
Cerebrospinal Fluid & 0.65 & 20.0 \\
\hline
\end{tabular}

\subsection{Electrical Impedance Tomography (EIT)}

The resistivity of human body tissues covers a wide range of values (A detailed table of resistivities for biological tissues is provided in [4]). Table 1 shows the resistivities of five different body tissues [4]. The corresponding X-ray attenuation coefficients are also given in the second column for comparison. It is clear that, the electrical resistivity changes from tissue to tissue. Furthermore, the resistivities of two tissues, whose $X$-ray attenuation coefficients are similar, can greatly vary [4], [78]. Therefore, those tissues which can not be discriminated using X-ray CT can be discriminated by resistivity images. This shows that an imaging system that reconstructs resistivity images can provide complementary information to the currently available imaging modalities such as X-ray CT. In Electrical Impedance Tomography (EIT), the spatial distribution of tissue resistivity is reconstructed. The data used in this reconstruction is obtained by applying sinusoidal $(50 \mathrm{kHz})$ currents from the body surface and measuring the resultant surface potential differences. In practice, finite number of electrodes are attached on the boundary of a cross section of a body segment (see Figure 1). A pair of electrodes is selected for current application and other electrode pairs are used for potential difference measurements [10]. By changing the current drive pair, different data sets are obtained and then used for image reconstruction. This data collection strategy is the most widely used strategy in EIT, however, there are other current drive and voltage measurement methods 


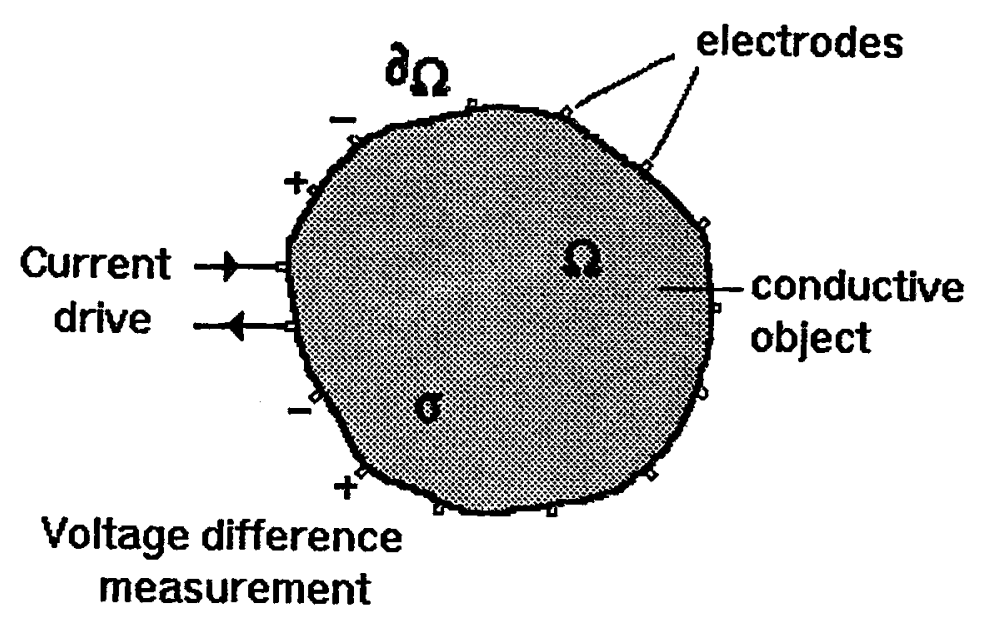

Figure 1.1. General principles of excitation and data collection in injected current EIT. $\sigma$ represents the conductivity distribution in the two-dimensional object $\Omega$. Finite number of electrodes are attached on the object boundary $\partial \Omega$ to apply current and measure the voltage differences.

in the literature [46], [33], [8].

Different tissues in human body have also different electrical permitivities. However, below $100 \mathrm{kHz}$ the conduction current is much greater than the displacement current for soft biological tissues and this leads most investigators to neglect the dielectric effects [5], [78], [9]. The tissue permitivity becomes effective at frequencies above about $200 \mathrm{kHz}$ but because of measurement difficulties (arising from the effect of stray wiring capacitance) these frequencies are not ususally used [5].

A major disadvantage of EIT with respect to other imaging modalities is its poor spatial resolution. It is known that, the number of independent measurements for an $\mathrm{N}$ electrode EIT system is $\mathrm{N}(\mathrm{N}-1) / 2$ [10], [46]. Therefore, 
in order to increase the spatial resolution in EIT images, the number of electrodes has to be increased. However, a typical data acquisition system use at most 32 electrodes because of measurement difficulties (practical and electronic) $[10],[46],[70]$. A second problem in EIT is that measurements are less sensitive to the conductivity perturbations in the interior regions, i.e. the small perturbations in the measurements can be caused by large perturbations in conductivity distribution [69], [47], [18], [20]. This property of EIT limits the resolution and accuracy in the reconstructed images.

EIT systems, however, use low cost PC based data collection hardware which may provide both anatomical and functional information (dynamic imaging is also possible using a real time data acquisition system) [72]-[74]. As a supplemental aid to other imaging modalities and with the above given advantages, EIT is a promising technique and studies on image reconstruction algorithms, data collection hardware and clinical applications still continue.

\subsection{Historical development of EIT}

The first attempts for imaging spatial distribution of tissue resistivity was presented by Henderson and Webster in 1978 [39]. They proposed an impedance camera by making use of a large electrode on the back of the thorax and $100(10 \times 10)$ electrodes on the front. Voltage was applied from the large electrode and currents flowing through the small electrodes on the front were measured. They applied guarded electrode technique to "straighten" the current streamlines but obtained low resolution transmission images of thorax.

In 1979, the following question was asked by Price [64]: Is it possible to make measurements on the exterior of the patient such that conductivity and permitivity distributions in a tomographic slice can be reconstructed? In his study, Price applied small voltage differences from the object boundary and measure the resulting small currents. He proposed a linear network approximation for the continuous resistivity distribution and his computer model lead him

$u \rightarrow$ to the basic characteristic of EIT, namely relatively less sensitivity of boundary measurements to inner region conductivity variations. He also pointed out the 
use of guarded electrodes for data collection.

In 1980, Dines and Lytle generated estimates of conductivity images by using an iterative process on linearized network equations for geophysical diagnostics of core samples using a voltage-drive current-measurement system [14].

Bates et al (1980) showed that, it was impossible (using the previous impedance computed tomography approaches) to straighten the current streamlines sufficiently and to reconstruct the conductivity distributions uniquely. They also demonstrated that, in order to obtain unambiguous images of a simple "onion-like" conductivity distribution, more comprehensive measurements had to be made [6].

In Schomberg and Tasto's approach (1981) (which was reported by several investigators [6], [5], [9]), images were reconstructed using X-ray CT techniques. In their study, a grid of small electrodes were used on one side of the object and a large electrode on the other side. The difference of the calculated and measured streamline resistances were back-projected along streamlines. This process was repeated for different object and electrode configuration orientations until the error between calculated and measured resistances reached a minimum [67].

In 1983, the first in vivo images were reconstructed by Barber et al [4] using a simple linear reconstruction process which was an adaptation of the method of back-projection used in X-ray CT [49] (equipotential lines method). In contrary to the former investigators, they proposed to apply current from the boundary and measure potential differences from the adjacent electrodes. Although the reconstructed image was quantitatively inaccurate, it was a good representation of the resistivity distribution of the forearm. In this study, they also pointed out the advantage of speed of their algorithm over iterative algorithms. It was also emphasised that using a very fast data collection system dynamic images of moving structures could be reconstructed. 
Kim et al (1983) developed a 3-D computer body model based on Finite Element Method and proposed two new electrode configurations for thorax imaging. In parallel-beam-like-projection the current streamlines were nearly parallel. In the second configuration, i.e. fan-beam-like-projection, the current streamlines were shown to spread like a fan at the voltage supplying electrode. The images of computer simulations were reconstructed by applying an iterative discrete back-projection algorithm (perturbation method). In this algorithm, the errors between the measured and calculated currents were back-projected by modifying each elements resistivity according to its present value and related sensitivity coefficient [Kim].

In a review article (1984), Barber and Brown summarized the previous studies performed for imaging spatial resistivity distribution of human body tissues. In this article, different data collection systems were presented. Image reconstruction algorithms were analyzed in many respects including uniqueness of solutions and three dimensional effects [5].

Tarassenko and Rolfe (1984) reported a new algorithm based on back-projection method proposed in [4], in which the ratios of measured to expected values were weighted with the appropriate sensitivity coefficients before being back-projected onto each conductjvity pixel. They compared the two algorithms using the two-dimensional (2-D) phantoms and pointed out the absence of "echoes" and increased contrast in their images [76].

Wexler et al in 1985 presented a novel approach for imaging the the conductivity distribution of three dimensional objects (double constraint algorithm). The reconstruction process do not require the assumption that the current forms beam-like paths while most of the previous investigators has accepted that this was a necessity. In this study, a three-dimensional (3-D) grid of nodes was defined over a cube including the region of interest while current injection and voltage measurement locations were assumed to be at the top surface. Two of the electrodes were selected for current injection and others were used for voltage measurement except the one selected as the reference point. By using Finite Element Method they solved the potential function twice, namely in the first one only Neumann boundary conditions were used and in the second one 
actual measurements were used as Drichlet boundary conditions while leaving the Neuman boundary conditions unchanged. The two solutions were expected to be same if the unknown conductivity distribution was used in calculations. Since conductivity distribution was just an estimate, a least squares technique was employed to produce an improved estimate that satisfies both boundary conditions for all excitations in the average sense. Computer simulation studied were presented for both 2-D and 3-D objects and 2-D experimental results were presented [79].

Murai and Kagawa (1985) presented simulation studies for 2-D models using Finite Element Method for the solution of potential distribution [53]. They applied Generalized Inversion method for image reconstruction to the linearized system of equations based on Geselowitz sensitivity theorem [31].

Isaacson in 1986 defined the term "distingushability" of two conductivity distributions when potential difference measurements were made by certain precision. He showed that the eigenfunctions of a linear operator corresponding to the largest eigenvalues were the "best" current patterns to be applied to distinguish two conductivities in the mean square sense [46]. He noted that, the boundary measurements were most sensitive to changes in conductivity far from the boundary if low (spatial) frequency currents were applied from the surface. The high (spatial) frequency currents would yield voltages which were mostly sensitive to conductivity perturbations close to the surface.

The first European Community workshop on EIT was held in Sheffield in 1986 and many valuable studies were presented on image reconstruction algorithms, data collection hardware and clinical applications. An issue of Clinical Physics and Physiological Measurements was devoted to the proceedings of this workshop (A list of the relevant works published in recent years was also given at the end of this issue). Some of the studies presented in this workshop are given below.

Seagar et al pointed out the theoritical limits to sensitivity and resolution and limitations to hardware design in impedance imaging. [69], [70]. 
Jossinet and Kardous presented their studies on investigating the sensitivity of measurements to conductivity perturbations by using a physical model. They noted that sensitivity was high in the proximity of electrodes and lower far from them [47].

Yorkey et al compared different reconstruction algorithms with 2-D computer simulations and showed the superiority of Newton Rampson method [80]. The algorithms compared were perturbation method, equipotential lines method, iterative equipotential lines method and double constraint method. They also pointed out the sensitivity of Newton Rampson Method to measurement errors.

Breckon and Pidcock discussed the mathematical aspects of impedance imaging . They comment on the non-linear and ill-posed nature of the inverse problem (i.e reconstructing conductivity distribution using boundary potential difference measurements). They also reported the recent studies of mathematicians on classes of conductivity distributions which can be uniquely identified by boundary measurements [9].

Powel et all considered [63] the use of a linear array of electrodes for imaging the related planar conductivity distribution using the back-projection algorithm proposed by Barber et al [4]. They reported that such an electrode configuration can be moved on the object easily and may be more usefull if data can be collected in real time.

In [11] and in that special issue of Clinical Physics and Physiological Measurement told above (1987), papers on some clinical applications of EIT like: assesing gastric function [57], early detection of intraventricular haemorrhage in the newborn [59], thermal monitoring of hyperthermia treatment [13], noninvasive temperature mapping in hyperthermia [32], monitoring respiration [37] and localization of cardiac related impedance changes in the thorax [17] were also presented.

In 1988, Newell et al reported the realization of an electronic system capable of applying the best current patterns to the periphery of the body. It 
was based on the ideas presented in [46] and [33] to distinguish the unknown conductivity from a conjectured conductivity. In that system there were 32 programmable current generators connected to 32 electrodes[61] .

In 1990, Ider et al reported a new algorithm for electrical impedance tomography of finite cylinders with general cross sectional boundaries and translationally uniform conductivities [45]. The method was applied to humans using Sheffield data collection protocol [10] and satisfactory images were presented.

The second, third and fourth European Community workshops were held in 1988, 1990 and 1991 and over than hundred papers were presented. (The papers presented in second and fourth workshops were again published in special issues of Clinical Phsics and Physiological measurement (Vol 9 and Vol 13).) The increased number of papers published in recent years shows that EIT is still a hot topic for the investigators studying on the new imaging modalities.

It can be argued that imaging the spatial distribution of tissue conductivity by boundary measurements is an exciting idea. However, although considerable effort has been spent for improving this imaging technique for the last 15 years, there are some basic problems like poor spatial resolution and inaccuracy in reconstructed images even when the problem is assumed to be 2-D. The theoretical limits related to these properties has also been studied by mathematicians. Breckon t al compiled and summarized these studies in [7].

As given in [7], Calderon (1980) had considered the uniqueness problem and showed that no two small changes in conductivity can produce the same boundary data [12]. He had given an explicit formula for the derivative $\left(F^{\prime}\right)$ of the forward mapping (i.e. $F$ maps conductivity to boundary measurements) and showed that $F^{\prime}$ was invertable but unfortunately not continuous. This result showed that small perturbations in boundary data may result from large perturbations in conductivity [12].

Kohn and Vogelius $(1984,1896)$ had proved that piecewise analytic conductivities in 2-D domain can be distinguished by boundary meaurements [51]-[53]. 
Sylvester and Uhlmann (1986) had showed that smooth conductivities (infinitely differentiable) can be distinguished by boundary measurements in a 3-D domain. They had also proved that smooth conductivity distributions sufficiently close to uniformity on a two dimensional domain can also be distinguished [75].

The results of mathematicians given above were obtained considering that boundary conditions have high spatial frequency content. However, Gisser et al showed that, in practice, increasing the number of electrodes to infinity (in order to obtain higher resolution reconstruction) leads signal-to-noise-ratio (SNR) to go to zero for single currents applied on a pair of electrodes [34]. They also noted that if "best" currents (current patterns which result in best distinguishability of two conductivity distributions by boundary measurements) were to be applied, in order to maximize the SNR, the electrodes should be as large as possible. They also presented an intuitive reasoning to this result. They noted that since "best" currents were smooth functions they can be better approximated by finite number of electrodes if there exist small gaps between the electrodes.

\subsection{EIT in Middle East Technical University}

The EIT studies in the Electrical and Electronics Engineering Department of Middle East Technical University has started by Y. Z. Ider and E. Atalar in 1985. In doing his M. Sc thesis, Atalar developed 2-D analytical and numerical solution methods for the solution of the forward problem of EIT under supervision of Ider. He developed a software to solve the equations obtained by Finite element Method using the Frontal algorithm and selected a proper mesh to provide the necessary accuracy in the solutions. Atalar and Ider then implemented the Iterative Equipotential Lines Method and applied using simulated data [2],[3]. In 1986, N. G. Gençer and C. Altan joined to this group and experimental studies started. A PC based data collection system is realized and images of real objects are presented by using the data collected from 2-D phantoms [1], [3], [41], [45]. In 1987, Atalar and Altan departured 
from the group and studies continue on the the development of a dual modality imaging system for impedance tomography with ultrasonically determined boundaries [42]. The ultrasonic sub-system is realized by Erkan Dorken under supervision of H. Köymen [15], [16]. Therafter, several Algebraic Reconstruction Techniques are applied for image reconstruction and resolution limits of EIT are investigated by Gençer and İder [19], [20], [22]. A 3-D FEM formulation is made and necessary softwares are prepared. The effects of off-plane conductivity distributions on the boundary data are analyzed numerically and experimentally and a correction method is developed [20], [21], [43]. For a short period of time $\mathrm{H}$. Tosun joined the group and studies continue on the electrical impedance tomography of finite cylinders with general cross sectional boundaries and translationally uniform conductivity distributions. The static images of human arm and thorax were obtained using the developed correction algorithm and presented in [45]. In 1990, B. Nakiboğlu and M. Kuzuoğlu joined to group and studies focused on eliminating the electrode position determination problem. The object to be imaged was assumed to be immersed into water

filled containers. Numerical and experimental results were obtained showing the effects of peripheral layer conductivity on the reconstructed images. The boundary of the immersed object is parametrized using Fourier Expansion and the shape determination techniques are developed to estimate the parameters of the boundary model [23], [25], [44], [60]. Since 1990, Gençer, İder and Kuzuoğlu continue their studies on image reconstruction algorithms for injected current EIT and on induced current EIT [24], [26]-[30], [55].

\subsection{EIT Using Induced Currents}

Induced current Electrical Impedance Tomography (EIT), has been proposed in 1990 as a new method for conductivity imaging of human tissues [65], [68]. Images from two and three dimensional phantoms have also been demonstrated [65], [68], [38]. Figure 2. demonstrates the general principles of excitation and data collection in induced current EIT for the two-dimensional (2-D) case. The conductive region $\Omega$, is surrounded by a current carrying conductor. The time varying magnetic field generated by the current in the coil (the primary magnetic field), induces current in the region $\Omega$. The resultant 


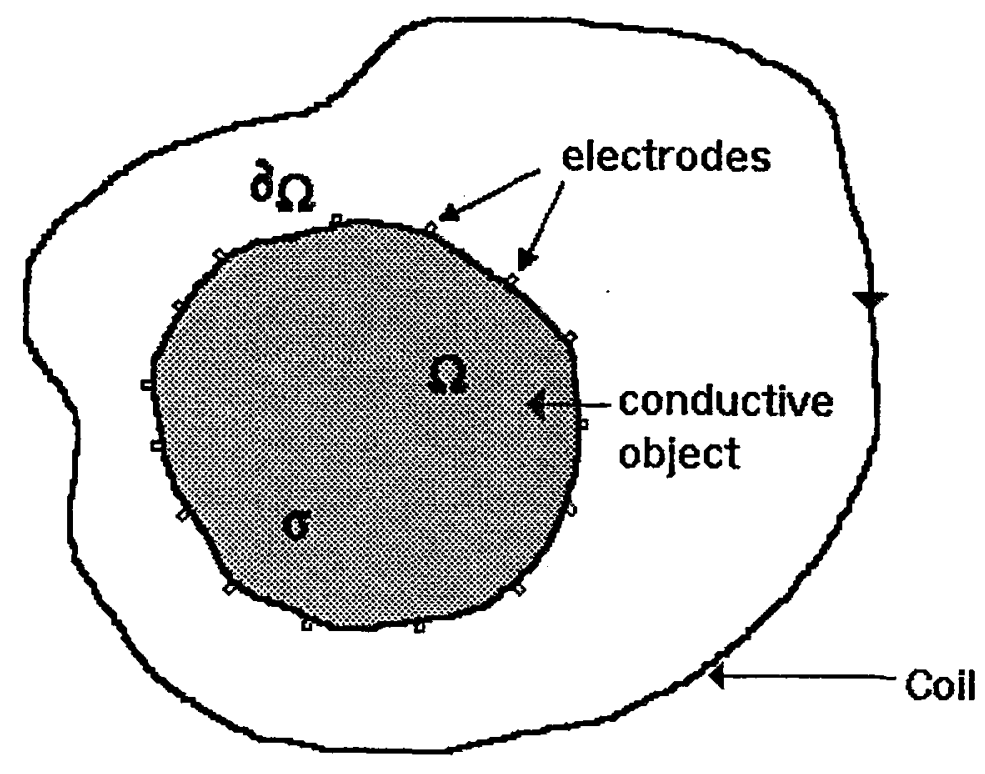

Figure 1.2. General principles of excitation and data collection in induced current EIT. $\sigma$ represents the conductivity distribution in the two-dimensional object $\Omega$. A current carrying coil is placed to induce currents in $\Omega$. Finite number of electrodes are attached on the object boundary $\partial \Omega$ to measure the voltage differences.

voltage differences between the finite number of electrodes on the surface $\partial \Omega$ are measured. By using different coil shapes or by changing the position of a given coil with respect to the object, one can generate different primary magnetic fields in $\Omega$. This allows one to increase the number of measurements. This new imaging technique is similar to conventional injected current EIT systems since the boundary potential differences are used for conductivity imaging in both methods. However, in injected current EIT, current is injected into $\Omega$ through the same surface electrodes whereas in induced current EIT current is induced in $\Omega$ by the time varying magnetic field. The use of current induction in the later method provides a flexibility in the number of electrodes attached to the surface of the object. In injected current EIT, the number of electrodes has to be increased in order to apply more independent current distributions inside the object [69], [10], [34]. However, in induced curent current EIT, it is evident that 
for a certain number of electrodes attached to the object surface, the number of independent current distributions introduced inside the object depends on the number of independent primary magnetic field distributions applied.

\subsection{The Objectives of this thesis}

Induced current EIT is not yet studied in detail in mathematical terms. This is necessary in order to investigate quantitatively the assumptions of the technique and its ultimate limitations, and also so that it can be compared to other similar techniques such as injected current EIT [4], [10]. The experimental

$\rightarrow$ studies given in [38], [65], [68] dogs not provide any details about the data collection hardware. These details are necessary in order to understand the feasibility of this method and so that it can be compared to the electronics used in injected current EIT. Therefore, the objectives of this thesis can be listed as given below.

1. To provide a mathematical formulation for the forward problem (i.e. calculation of measurements for a given conductivity distribution).

2. To provide numerical solution methods for the forward problem.

3. To analyze the sensitivity of measurements to pixel conductivity perturbations.

4. To formulate the inverse problem (i.e. calculation of unknown conductivity distribution) and analyze its ill-posedness.

5. To prepare a numerical solution method for the inverse problem and reconstruct images of simulated data.

6. To develop a PC based data collection hardware and test the validity of theoretical approaches developed in this study. To reconstruct images using real data collected from 2-D phantoms.

7. To propose an alternative coil configuration in order to improve the performance of induced current EIT. 


\subsection{Structure of this Thesis}

In Chapter 2, the forward problem is formulated by deriving the partial differential equations and boundary conditions. The relation between the actual measurements and the functions defined by these partial differential equations are also given in this chapter. Chapter 3 includes the numerical fromulations developed for the solution of the forward problem. The numerical solutions obtained by simulated data are presented and a linear system of equations is obtained relating the perturbations in conductivity to perturbations in measurements. The inverse problem is analyzed in Chapter 4 . The sensitivity of measurements to pixel conductivities, ill-posedness of the inverse problem and the issue of number of independent measurements are investigated in detail. The images obtained using simulated data are presented and an alternative coil configuration is suggested to improve the performance of induced current EIT. In Chapter 5, the details of the developed prototype data acquisition hardware are explained. Experimental results obtained by using real data are presented. 


\section{CHAPTER II}

\section{FORMULATION OF THE FORWARD PROBLEM}

\subsection{Introduction}

In this section, first the basic field equations governing the behaviour of sinusoidally varying electromagnetic fields in a conductive medium will be given. Secondly, the general formulation of the partial differential equations governing the scalar and vector potential functions will be presented from which the electric field intensity and the magnetic field density can be derived. Thereafter, relation of measurements to scalar and vector potential functions will be described.

\subsection{Basic Field Equations}

The following set of Maxwell's equations govern the behaviour of sinosoidally varying ( $e^{j \omega t}$ time-dependence is assumed) electromagnetic fields in a linear, non-magnetic, isotropic conductive medium [62]:

$$
\begin{aligned}
\nabla \times \vec{E} & =-j \omega \vec{B} \\
\nabla \times \vec{B} & =\mu_{0}(\sigma+j \omega \epsilon) \vec{E} \\
\nabla \cdot \vec{D} & =\rho \\
\nabla \cdot \vec{B} & =0
\end{aligned}
$$

with the continuity equation

$$
\nabla \cdot \vec{J}=-j \omega \rho
$$

where $\vec{D}$ and $\vec{J}$ are related to $\vec{E}$ by

$$
\vec{J}=\sigma \vec{E}
$$




$$
\vec{D}=\epsilon \vec{E}
$$

As a consequence of equation (2.4), it is possible to introduce the magnetic vector potential [62] $\vec{A}$ defined by

$$
\vec{B}=\nabla \times \vec{A}
$$

and using equation (2.1), one can find an expression for the electric field intensity $\vec{E}$ as

$$
\vec{E}=-\nabla \phi-j \omega \vec{A}
$$

where $\phi$ is defined as the scalar potential [62].

2.3 Formulation of the Partial Differential Equations governed by Scalar and Magnetic Vector Potential Functions

The continuity equation given by equation (2.5) can be rewritten using equations (2.3) and (2.7) as

$$
\nabla \cdot(\vec{J}+j \omega \epsilon \vec{E})=0
$$

where the term $j \omega \epsilon \vec{E}$, is referred to as the displacement current density. Using equation (2.6) and (2.9), equation (2.10) becomes

$$
\nabla \cdot[(\sigma+j \omega \epsilon) \nabla \phi]=-j \omega \vec{A} \cdot \nabla(\sigma+j \omega \epsilon)
$$

provided that the condition $\nabla \cdot \vec{A}=0$ is adopted. This particular choice (i.e, $\nabla \cdot \vec{A}=0$ ) is known as Coulomb's gauge in the literature [48], [54]. The reason for this freedom in specifying the divergence of $\vec{A}$ is that, $\vec{A}$ is an auxiliary function and only its curl is related to an observable physical quantity (i.e, $\vec{B}=\nabla \times \vec{A})$. On the other hand $\nabla \cdot \vec{A}$ must also be specified in order to completely specify $\vec{A}[62]$. The Coulomb gauge is preferred when conductivity distribution is not uniform [54]. The boundary condition of the above partial differential equation can be found by equating the normal component of the total current on $\partial \Omega$ to zero to obtain

$$
\frac{\partial \phi}{\partial n}=-j \omega \vec{A} \cdot \vec{n}
$$


where $\vec{n}$ represents the outward unit normal vector. Taking the curl of equation (2.8) and using equations (2.2) and (2.9), one may obtain the following partial differential equation

$$
\nabla \times(\nabla \times \vec{A})=\mu_{0}(\sigma+j \omega \epsilon)(-\nabla \phi-j \omega \vec{A})
$$

or using the vector identity $\nabla \times(\nabla \times \vec{A})=\nabla^{2} \vec{A}-\nabla(\nabla \cdot \vec{A})$ and again using Coulomb's gauge,

$$
\nabla^{2} \vec{A}=\mu_{0}(\sigma+j \omega \epsilon)(-\nabla \phi-j \omega \vec{A})
$$

Equations (2.11) and (2.14) are the coupled partial differential equations which have to be handled together for the solution of $\phi$ and $\vec{A}$ in $\Omega$. However, by means of the following two assumptions the problem of finding $\phi$ is considerably simplified. These assumptions are : 1) the total magnetic vector potential $\vec{A}$ is approximately equal to the primary vector potential $\vec{A}_{p}$ which exists in

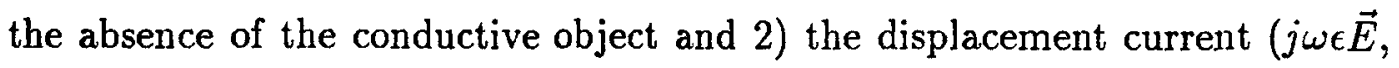
where $\omega=2 \pi f, f=50 \mathrm{kHz}$ ) is negligible compared to the conduction current [5],[9] (See Appendix $\mathrm{C}$ for the validation of these assumptions). Using these assumptions, equations $(2.11)$ and $(2.12)$ reduce to

$$
\begin{aligned}
\nabla \cdot(\sigma \nabla \phi) & =-j \omega \vec{A}_{p} \cdot \nabla \sigma \\
\frac{\partial \phi}{\partial n} & =-j \omega \vec{A}_{p} \cdot \vec{n}
\end{aligned}
$$

or by decomposing $\phi$ into its real $\left(\phi_{R}\right)$ and imaginary $\left(\phi_{I}\right)$ parts

$$
\begin{aligned}
\nabla \cdot\left(\sigma \nabla \phi_{R}\right) & =0 \\
\frac{\partial \phi_{R}}{\partial n} & =0 \\
\nabla \cdot\left(\sigma \nabla \phi_{I}\right) & =-\omega \vec{A}_{p} \cdot \nabla \sigma \\
\frac{\partial \phi_{I}}{\partial n} & =-\omega \vec{A}_{p} \cdot \vec{n}
\end{aligned}
$$

From equations (2.17) and (2.18) it is seen that $\phi_{R}$ is zero. Therefore, using $\phi$ and $\vec{A}$ in place of $\phi_{I}$ and $\vec{A}_{p}$ for notational simplicity, calculation of the scalar potential distribution in $\Omega$ for a certain coil configuration requires the solution of the following partial differential equation

$$
\begin{aligned}
\nabla \cdot(\sigma \nabla \phi) & =-\omega \vec{A} \cdot \nabla \sigma & & \text { in } \Omega \\
\frac{\partial \phi}{\partial n} & =-\omega A_{n} & & \text { on } \partial \Omega
\end{aligned}
$$


where $A_{n}$ represents the normal component of $\vec{A}$ on $\partial \Omega$. Calculation of the primary magnetic vector potential for any coil configuration can be done as shown in Appendix A.

Since the object must remain electrically neutral, the net rate of increase of volume and surface charges in $\Omega$ and on $\partial \Omega$ has to be equal to zero. This condition can be expressed mathematically as

$$
-\int_{\Omega} \nabla \cdot(\sigma \nabla \phi) d \Omega+\oint_{\partial \Omega} \sigma \frac{\partial \phi}{\partial n} d(\partial \Omega)=0
$$

from which one may show the consistency of equations (2.21) and (2.22) since

$$
\int_{\Omega} \nabla \cdot(\sigma \omega \vec{A}) d \Omega-\oint_{\partial \Omega} \sigma \omega A_{n} d(\partial \Omega)=0
$$

using Gauss' theorem [62].

Clearly, a solution of the Neumann problem is not unique, since given $\phi, \phi$ plus any constant will also satisfy the stated conditions. However, if the value of $\phi$ at a point in $\Omega$ or or on $\partial \Omega$ is specified the solution will be unique.

\subsection{Relation of actual measurements to $\phi$ and $\vec{A}$}

The actual measurements are the voltage differences measured between electrodes placed on the surface. The voltage difference between two points on the surface $a$ and $b$, i.e. $v_{a b}$ is the sum of three terms.

$$
v_{a b}=-\int_{a}^{b} \nabla \phi \cdot d \vec{l}-\int_{a}^{b} j \omega \vec{A} \cdot d \vec{l}+E M F_{\text {loop }}
$$

where, the line integrals can be taken on any line in $\Omega$ connecting $a$ and $b$, and the $E M F_{\text {loop }}$ is the voltage induced in the loop formed by the line between $a$ and $b$ and the measurement cables. If the conductivity distribution is perturbed, only the first term changes (remember the first assumption). Thus, perturbation in $v_{a b}$, following a perturbation in conductivity distribution, depends only on the perturbation in $\nabla \phi$ field. Indeed, in practice, because of the difficulties in precisely controlling the $E M F_{\text {loop }}$ term which depends on how the electrode 
cables are placed, perturbation in $v_{a b}, \Delta v_{a b}=-\int_{a}^{b} \Delta(\nabla \phi) \cdot d \vec{l}$, is measured. However, this brings the limitation that, only the conductivity perturbation compared to a reference can be imaged.

The relation between $\sigma$ and $\phi$ is a rather complicated non-linear mapping. However, the first order variation in the scalar potential function $(\Delta \phi)$ related to the perturbation $(\Delta \sigma)$ in the conductivity distribution can be determined. If $\phi_{0}$ and $\phi$ are the potential distributions corresponding to $\sigma_{0}$ and $\sigma$, respectively, they obey the following partial differential equations :

$$
\begin{aligned}
\nabla \cdot\left(\sigma_{0} \nabla \phi_{0}\right) & =-\omega \vec{A} \cdot \nabla \sigma_{0} \\
\nabla \cdot(\sigma \nabla \phi) & =-\omega \vec{A} \cdot \nabla \sigma
\end{aligned}
$$

Equation (2.24) can be rewritten, by substituting $\phi_{0}+\Delta \phi$ and $\sigma_{0}+\Delta \sigma$ for $\phi$ and $\sigma$.

$\nabla \cdot\left(\sigma_{0} \nabla \phi_{0}\right)+\nabla \cdot\left(\sigma_{0} \nabla(\Delta \phi)\right)+\nabla \cdot\left(\Delta \sigma \nabla \phi_{0}\right)+\nabla \cdot[\Delta \sigma(\nabla(\Delta \phi))]=-\omega \vec{A} \cdot \nabla \sigma_{0}-\omega \vec{A} \cdot \nabla(\Delta \sigma)$

The last term in the left hand side can be neglected since it is a second order variation. The first terms of both sides can be dropped from the equation since they are equal (given by equation (2.23)). Therefore,

$$
\begin{gathered}
\nabla \cdot\left(\sigma_{0} \nabla(\Delta \phi)\right)=-\nabla \cdot\left(\Delta \sigma \nabla \phi_{0}\right)-\omega \vec{A} \cdot \nabla(\Delta \sigma) \\
\frac{\partial(\Delta \phi)}{\partial n}=0
\end{gathered}
$$

These equations relate the perturbation in $\phi$ to the perturbation in $\sigma$. It is apparent that the source of $\Delta \phi$ is related to the gradient of $\Delta \sigma$. For a simpler case for which $\sigma_{0}$ is constant

$$
\sigma_{0} \nabla^{2}(\Delta \phi)=-\nabla \sigma \cdot\left(\nabla \phi_{0}+\omega \vec{A}\right)
$$

is obtained. Thus, the regions where $\sigma$ varies, are regions of charge accumulation and this accumulated charge generates the $\Delta \phi$ field. The strength of the source depends on $\nabla \sigma$ as well as the $\vec{E}$ field $\left(\vec{E}=\nabla \phi_{0}+\vec{A}\right)$ obtained for the reference conductivity distribution $\left(\sigma_{0}\right)$.

In practice, integral of $\Delta(\nabla \phi)$ between measurement electrodes are measured. Since $\nabla \phi$ is irrotational [62], with 16 electrodes, one can only make 
15 independent measurements. Thus, if $M$ different primary magnetic fields are used, one can obtain a maximum of $15 \mathrm{M}$ independent measurements. 


\section{CHAPTER III}

\section{NUMERICAL FORMULATION AND SOLUTION OF THE FORWARD PROBLEM}

\subsection{The Series Expansion Method (SEM) for solving $\phi$}

The Series Expansion Method can be used to solve the scalar potential in 2-D objects with a circular cross section and with arbitrary number of concentric layers having different conductivities. In this section, two cases are treated, namely a 2-D circular object with uniform conductivity (Figure 3.1) and a 2-D circular object with two concentric layers of different conductivities (Figure 3.2).

\subsubsection{2-D circular object with uniform conductivity}

When the conductivity is uniform inside the object as shown in Figure 3.1., the scalar potential equation given by equations (2.21) and (2.22) reduces to

$$
\begin{aligned}
\nabla^{2} \phi & =0 & & \text { in } \Omega \\
\frac{\partial \phi}{\partial n} & =-\omega A_{n} & & \text { on } \partial \Omega
\end{aligned}
$$

which is independent of the conductivity of the object. Equation (3.1) and (3.2) is the Laplace's Equation with Neumann boundary conditions for which a general solution can be found [62] as

$$
\phi(r, \theta)=\sum_{m=1}^{\infty} r^{m}\left(a_{m} \operatorname{Cos}(m \theta)+b_{m} \operatorname{Sin}(m \theta)\right)
$$

where $r$ is the radial distance from the origin and $\theta$ is defined as the angle with respect to positive $x$ axis. For a circular object of radius $R$, the coefficients $a_{m}$ 


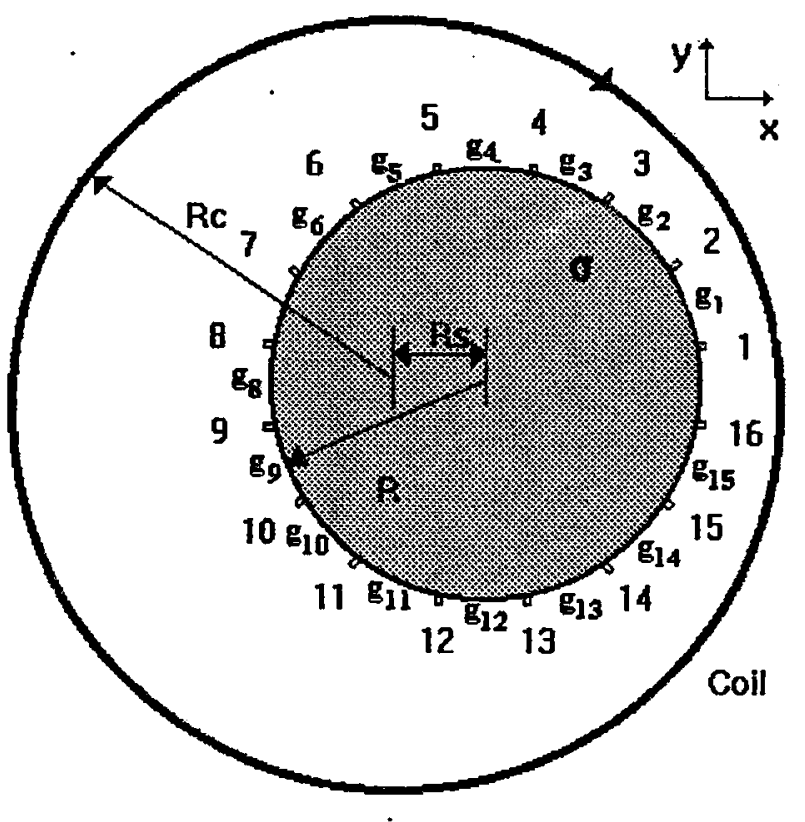

Figure 3.1. A circular object of radius $R$ placed inside a circular coil of radius $R_{c}$. $R_{s}$ denotes the coil center shift with respect to object origin. The object has uniform conductivity distribution $\sigma .16$ electrodes are placed around the object boundary. The numbering of the electrodes and related scalar poten$\rightarrow$ tial differences $\left(g_{1}, g_{2}, \ldots, g_{1 p}^{5}\right)$ are also shown along the object boundary. $g_{k}$ $(k=1, \ldots, 15)$ represents the difference of scalar potentials between the $(k+1)^{t h}$ and $k^{\text {th }}$ electrodes.

and $b_{m}$ can be found as

$$
\begin{aligned}
& a_{m}=-\frac{\omega}{m \pi R^{m-1}} \int_{0}^{2 \pi} A_{n}(\theta) \operatorname{Cos}(m \theta) d \theta \\
& b_{m}=-\frac{\omega}{m \pi R^{m-1}} \int_{0}^{2 \pi} A_{n}(\theta) \operatorname{Sin}(m \theta) d \theta
\end{aligned}
$$




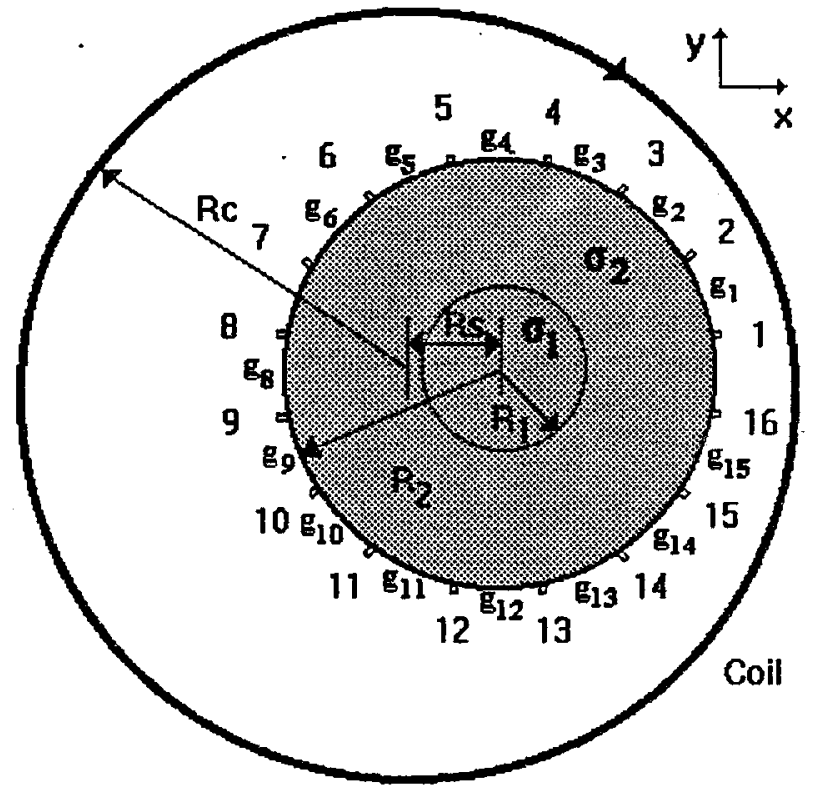

Figure 3.2. A circular object of radius $R_{2}$ placed inside a circular coil of radius $R_{c}$. The object has two concentric layers of conductivities $\sigma_{1}$ and $\sigma_{2}$. The radius of the central inhomogeneity is $R_{1}$.

\subsubsection{2-D circular object with a concentric inhomogeneity}

For the geometry illustrated in Figure 3.2, the solution for scalar potential function can be provided by expressing $\phi=\phi_{1}$ for $0 \leq r \leq R_{1}$ and $\phi=\phi_{2}$ for $R_{1} \leq r \leq R_{2}$ where

$$
\begin{aligned}
& \phi_{1}(r, \theta)=\sum_{m=1}^{\infty} r^{m}\left(e_{m} \operatorname{Cos}(m \theta)+f_{m} \operatorname{Sin}(m \theta)\right) \\
& \phi_{2}(r, \theta)=\sum_{m=1}^{\infty}\left(r^{m} a_{m}+r^{-m} b_{m}\right) \operatorname{Cos}(m \theta)+\left(r^{m} c_{m}+r^{-m} d_{m}\right) \operatorname{Sin}(m \theta)
\end{aligned}
$$

The necessary boundary and interface conditions are :

$$
\begin{aligned}
\left.\frac{\partial \phi_{2}}{\partial r}\right|_{r=R_{2}} & =-\omega A_{n}\left(R_{2}\right) \\
\left.\left(\sigma_{2} \frac{\partial \phi_{2}}{\partial r}-\sigma_{1} \frac{\partial \phi_{1}}{\partial r}\right)\right|_{r=R_{1}} & =-\left(\sigma_{2}-\sigma_{1}\right) \omega A_{n}\left(R_{1}\right) \\
\phi_{1}\left(R_{1}\right) & =\phi_{2}\left(R_{1}\right)
\end{aligned}
$$


The coefficients $a_{m}, b_{m}, c_{m}, d_{m}, e_{m}$ and $f_{m}$ can found as given below.

$$
\begin{aligned}
a_{m} & =\frac{1}{2}\left[(1+\alpha) e_{m}+(1-\alpha) \frac{1}{R_{1}^{m-1}} E(m)\right] \\
b_{m} & =\frac{1}{2}\left[(1-\alpha) R_{1}^{2 m} e_{m}-(1-\alpha) R_{1}^{m+1} E(m)\right] \\
c_{m} & =\frac{1}{2}\left[(1+\alpha) f_{m}+(1-\alpha) \frac{1}{R_{1}^{m-1}} F(m)\right] \\
d_{m} & =\frac{1}{2}\left[(1-\alpha) R_{1}^{2 m} f_{m}-(1-\alpha) R_{1}^{m+1} F(m)\right] \\
e_{m} & =\frac{2 \beta^{m} C(m)-\left[(1-\alpha)+(1-\alpha) \beta^{2 m}\right] \beta E(m)}{\beta^{m} R_{2}^{m-1}\left[(1+\alpha)-(1-\alpha) \beta^{2 m}\right]} \\
f_{m} & =\frac{2 \beta^{m} D(m)-\left[(1-\alpha)+(1-\alpha) \beta^{2 m}\right] \beta F(m)}{\beta^{m} R_{2}^{m-1}\left[(1+\alpha)-(1-\alpha) \beta^{2 m}\right]}
\end{aligned}
$$

where

$$
\begin{gathered}
\alpha=\frac{\sigma_{1}}{\sigma_{2}} \\
\beta=\frac{R_{1}}{R_{2}}
\end{gathered}
$$

and

$$
\begin{aligned}
& C(m)=-\frac{\omega}{\pi m} \int A_{n}\left(R_{2}, \theta\right) \operatorname{Cos}(m \theta) d \theta \\
& D(m)=-\frac{\omega}{\pi m} \int A_{n}\left(R_{2}, \theta\right) \operatorname{Sin}(m \theta) d \theta \\
& E(m)=-\frac{\omega}{\pi m} \int A_{n}\left(R_{1}, \theta\right) \operatorname{Cos}(m \theta) d \theta \\
& F(m)=-\frac{\omega}{\pi m} \int A_{n}\left(R_{1}, \theta\right) \operatorname{Sin}(m \theta) d \theta
\end{aligned}
$$

Obviously, the problem of finding the potential distribution for uniform conductivity distribution is a special case of the problem presented here, with $\alpha$ and $\beta$ taken to be equal to 1 . Calculation of the primary magnetic vector potential for a circular coil is given in Appendix A.

3.2 Formulation and Solution of the Forward Problem using Finite Element Method (FEM)

For a general conductivity distribution $\sigma$, equations (2.21) and (2.22) can not be handled by SEM. Hence, the FEM is used to solve the scalar potential function. For this reason, the region to be imaged is divided into 1016 
triangular elements corresponding to 541 nodes as shown in Figure 3.3 [2], [45]. On each element, conductivity is taken to be constant and $\phi$ is approximated by a summation of simple shape functions and the nodal values of $\phi$ are assumed to be the unknowns. If $\bar{V}$ denotes the $541 \times 1$ vector of unknown scalar potentials at the nodes, the following matrix equation has to be solved:

$$
S(\bar{\sigma}) \bar{V}=\bar{b}(\bar{\sigma})
$$

where $\bar{\sigma}$ denotes the $1016 \times 1$ vector of element conductivities. The details of this matrix equation for a simple mesh are given in Appendix B. In this equation, $S$ is a sparse $541 \times 541$ matrix whose entries depend on the element geometries and element conductivities and $\bar{b}$ is the $541 \times 1$ vector incorporating interface conditions as well as boundary conditions. Note that incorporation of the boundary and interface conditions into the vector $\bar{b}$ is not trivial and is shown in detail in Appendix B.

The solution for the scalar potential distribution is

$$
\bar{V}=S^{-1}(\bar{\sigma}) \bar{b}(\bar{\sigma})
$$

provided that the corresponding row and column of $S$ and $\bar{b}$ are modified to specify the potential value of a reference node. In this study the central node is chosen to be the reference node with zero potential. For a 16 electrode system, the $15 \times 1$ vector of surface scalar potential differences is denoted by $\bar{g}$ (see Fig 3.1 for definition of $\bar{g}$ ) and related to $\bar{V}$ through a matrix $C$ by the equation

$$
\bar{g}=C \bar{V}
$$

In order to obtain an equation relating the perturbation in $\bar{g}$ (i.e, $\Delta \bar{g}$ ) to the perturbation in $\bar{\sigma}$ (i.e, $\Delta \bar{\sigma}$ ), equation (2.26). is rewritten using $\sigma-\sigma_{0}$ in place of $\Delta \sigma$, as

$$
\nabla \cdot\left(\sigma_{0} \nabla(\Delta \phi)=-\left[\nabla \cdot\left(\sigma \nabla \phi_{0}\right)-\nabla \cdot\left(\sigma_{0} \nabla \phi_{0}\right)\right]-\omega\left[\vec{A} \cdot \nabla \sigma-\vec{A} \cdot \nabla \sigma_{0}\right]\right.
$$

The discretized version of the above equation using FEM can be written as

$$
S\left(\bar{\sigma}_{0}\right) \Delta \bar{V}=-\left[S(\bar{\sigma}) \bar{V}_{0}-S\left(\bar{\sigma}_{0}\right) \bar{V}_{0}\right]-\left[\bar{b}(\bar{\sigma})-\bar{b}\left(\bar{\sigma}_{0}\right)\right]
$$

where $\bar{\sigma}_{0}$ denotes the vector of initially assumed conductivities of elements and $\bar{V}_{0}$ is the vector of nodal values of scalar potential distribution solved for $\bar{\sigma}_{0}$. 


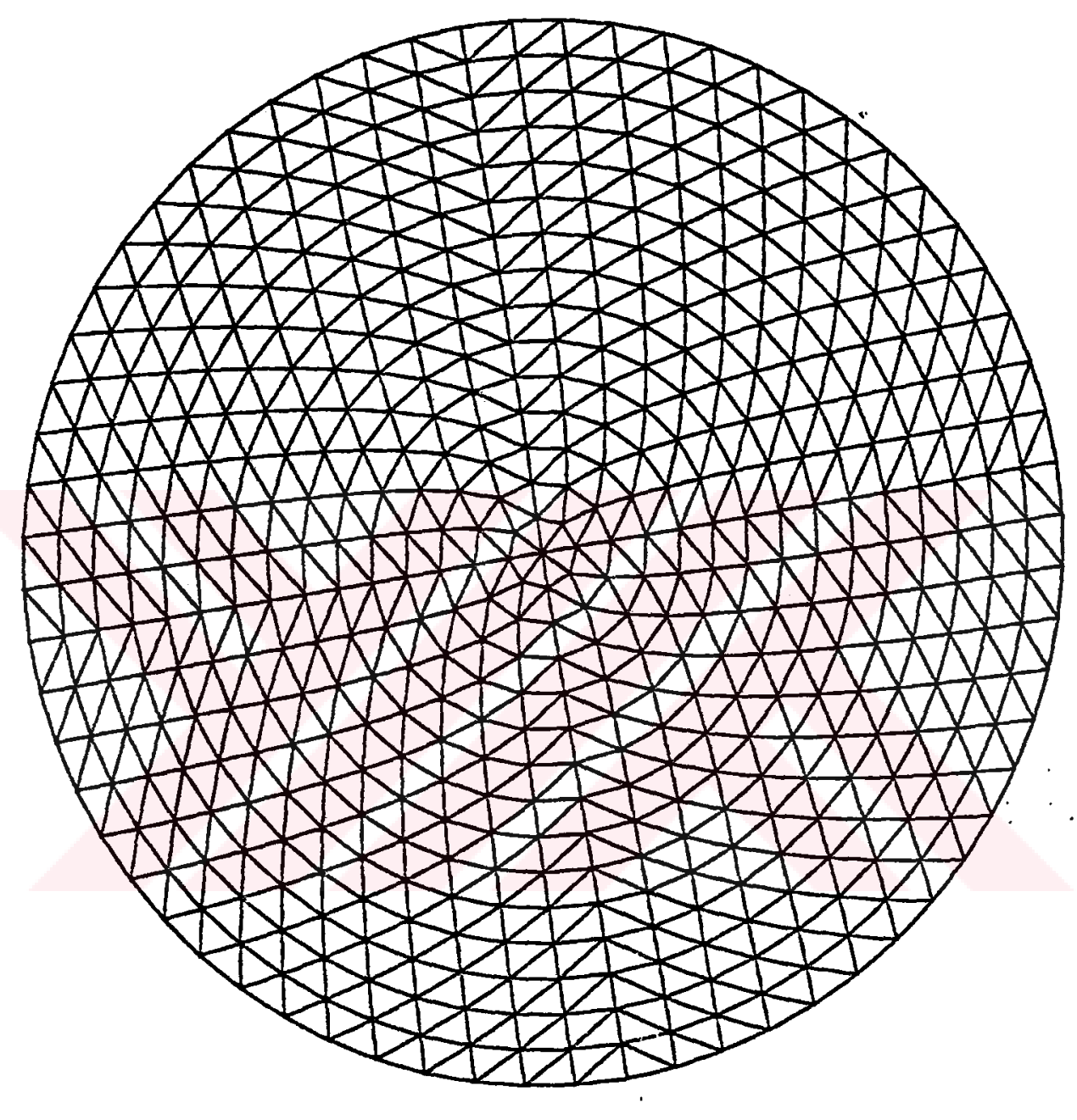

Figure 3.3. The 541-node, 1016-element mesh for FEM formulations 
This is equivalent to,

$$
S\left(\bar{\sigma}_{0}\right) \Delta \bar{V}=-\left.\frac{\partial}{\partial \bar{\sigma}}\left[S(\bar{\sigma}) \bar{V}_{0}+\bar{b}(\bar{\sigma})\right]\right|_{\bar{\sigma}=\bar{\sigma}_{0}} \Delta \bar{\sigma}
$$

Therefore, the linear system of equations relating $\Delta \bar{\sigma}$ to $\Delta \bar{V}$ is

$$
\Delta \bar{V}=-\left.S\left(\bar{\sigma}_{0}\right)^{-1} \frac{\partial}{\partial \bar{\sigma}}\left[S(\bar{\sigma}) \bar{V}_{0}+\bar{b}(\bar{\sigma})\right]\right|_{\bar{\sigma}=\bar{\sigma}_{0} \Delta \bar{\sigma}}
$$

Equation (3.29) is multiplied from left by the matrix $C$ (given in equation (3.25)) and

$$
\Delta \bar{g}=-\left.C S\left(\bar{\sigma}_{0}\right)^{-1} \frac{\partial}{\partial \bar{\sigma}}\left[S(\bar{\sigma}) \bar{V}_{0}+\bar{b}(\bar{\sigma})\right]\right|_{\bar{\sigma}=\bar{\sigma}_{0}} \Delta \bar{\sigma}
$$

is obtained. Similar expressions can be obtained for $M$ different primary magnetic fields. By introducing the index $j$ to clarify this point, the overall system of equations can be shown to be

$$
\Delta \bar{G}=\left[\begin{array}{c}
\Delta \bar{g}^{1} \\
\Delta \bar{g}^{2} \\
\cdot \\
\cdot \\
\cdot \\
\Delta \bar{g}^{M}
\end{array}\right]=-\left[\begin{array}{c}
\left.C S^{-1}\left(\bar{\sigma}_{0}\right) \frac{\partial}{\partial \bar{\sigma}}\left[S(\bar{\sigma}) \bar{V}_{0}^{1}+\bar{b}^{1}(\bar{\sigma})\right]\right|_{\bar{\sigma}=\bar{\sigma}_{0}} \\
\left.C S^{-1}\left(\bar{\sigma}_{0}\right) \frac{\partial}{\partial \bar{\sigma}}\left[S(\bar{\sigma}) \bar{V}_{0}^{2}+\bar{b}^{2}(\bar{\sigma})\right]\right|_{\bar{\sigma}=\bar{\sigma}_{0}} \\
\cdot \\
\cdot \\
\cdot \\
\left.C S^{-1}\left(\bar{\sigma}_{0}\right) \frac{\partial}{\partial \bar{\sigma}}\left[S(\bar{\sigma}) \bar{V}_{0}^{M}+\bar{b}^{M}(\bar{\sigma})\right]\right|_{\bar{\sigma}=\bar{\sigma}_{0}}
\end{array}\right] \Delta \bar{\sigma}
$$

Denoting the matrix on the right hand side as $R$ (the negative sign is included in $R$ ), the following equation is obtained :

$$
\Delta \bar{G}=R \Delta \bar{\sigma}
$$

where $R$ is named as the sensitivity matrix of dimension $15 M \times 1016$.

\subsection{Numerical Results}

3.3.1 Comparison of scalar potential solutions obtained by FEM and SEM

The accuracy of the scalar potential solutions using FEM are tested by comparing them with the solutions obtained using SEM. In order to determine the number of terms to be used in SEM equations for the solution of $\phi$ 
along $\partial \Omega$, the contribution of each new term to the series must be taken into account. In this study, solutions are terminated after the term whose maximum contribution to the sum is less than $1 \%$. The scalar potential distributions along $\partial \Omega$ for any coil configuration is calculated first for an object of uniform conductivity $(0.2$ Siemens $/ \mathrm{m})$ and then for another defined by parameters $\alpha=10$, $\beta=0.33, \sigma_{2}=0.2$ Siemens $/ \mathrm{m}$. The gradients obtained for these two objects are calculated by SEM and FEM and are given in Table 3.1. The coil used in this study is circular and has a radius of $0.36 \mathrm{~m}$. It is assumed to be shifted by $0.20 m$ towards positive $x$ axis with respect to the object origin. It is observed that the gradients are correct to within $1 \%$ (These and other numerical results presented in this paper are obtained by Pascal code and implemented in SUN $3 / 150$ workstation. In each simulation study, the coil current is assumed to be 1 Ampere ).

\subsubsection{Calculation of induced current components by FEM}

Since the potential distribution on each element is linearly approximated, the currents introduced by the scalar potential term of the electric field expression, i.e $-\sigma \nabla \phi$ is constant on each triangular element and can easily be calculated. The contribution of the changing magnetic field on the induced currents, i.e $-\sigma \omega \vec{A}$ can also be obtained for each element as $\vec{A}$ is calculated at the center of elements and assumed to be constant on each element. Therefore, the total current density on the $i^{\text {th }}$ element is.

$$
J_{i}=-\sigma_{i} \nabla \phi^{i}-\sigma_{i} \omega \vec{A}^{i} \quad i=1 \cdots 1016
$$

The distribution of current density is calculated on a circular object of radius $0.12 m$ for two different coil locations. The object is assumed to have a uniform conductivity of 0.2 siemens $/ \mathrm{m}$. A circular coil is first shifted towards positive $x$ axis, then towards positive $y$ axis with respect to object center. In each case, the two components of induced current density are calculated and separately displayed ((a) and ((b) parts of Figure 3.4 and 3.5). The current density components, individualy, seem to cross the object boundary. However, the expected circulatory behaviour of the total current densities are observed in Figure 3.4 (c) and 3.5 (c). 
Table 3.1. Comparison of Boundary Potential differences calculated by SEM and FEM for both uniform and non-uniform object distributions.

\begin{tabular}{||c|c|c|c|c||}
\hline Boundary & \multicolumn{2}{|c|}{ SEM } & \multicolumn{2}{c|}{ FEM } \\
\cline { 2 - 5 } Scalar & $(m V)$ & $(m V)$ & $(m V)$ & $(m V)$ \\
potential & $\alpha=1$ & $\alpha=10$ & $\alpha=1$ & $\alpha=10$ \\
differences & $\beta=1$ & $\beta=0.33$ & $\beta=1$ & $\beta=0.33$ \\
\hline$g_{1}$ & 2.7205 & 2.6849 & 2.7202 & 2.6845 \\
$g_{2}$ & 2.2253 & 2.1971 & 2.2247 & 2.1968 \\
$g_{3}$ & 1.4245 & 1.4079 & 1.4240 & 1.4074 \\
$g_{4}$ & 0.3600 & 0.3581 & 0.3589 & 0.3568 \\
$g_{5}$ & -0.8922 & -0.8783 & -0.8931 & -0.8791 \\
$g_{6}$ & -2.1880 & -2.1598 & -2.1876 & -2.1596 \\
$g_{7}$ & -3.2523 & -3.2141 & -3.2509 & -3.2128 \\
$g_{8}$ & -3.6833 & -3.6414 & -3.6800 & -3.6380 \\
$g_{9}$ & -3.2523 & -3.2141 & -3.2509 & -3.2128 \\
$g_{10}$ & -2.1880 & -2.1598 & -2.1876 & -2.1596 \\
$g_{11}$ & -0.8922 & -0.8783 & -0.8931 & -0.8791 \\
$g_{12}$ & 0.3600 & 0.3581 & 0.3589 & 0.3568 \\
$g_{13}$ & 1.4245 & 1.4079 & 1.4240 & 1.4074 \\
$g_{14}$ & 2.2253 & 2.1971 & 2.2247 & 2.1968 \\
$g_{15}$ & 2.7205 & 2.6849 & 2.7202 & 2.6845 \\
$g_{16}$ & 2.8878 & 2.8797 & 2.8876 & 2.8495 \\
\hline
\end{tabular}



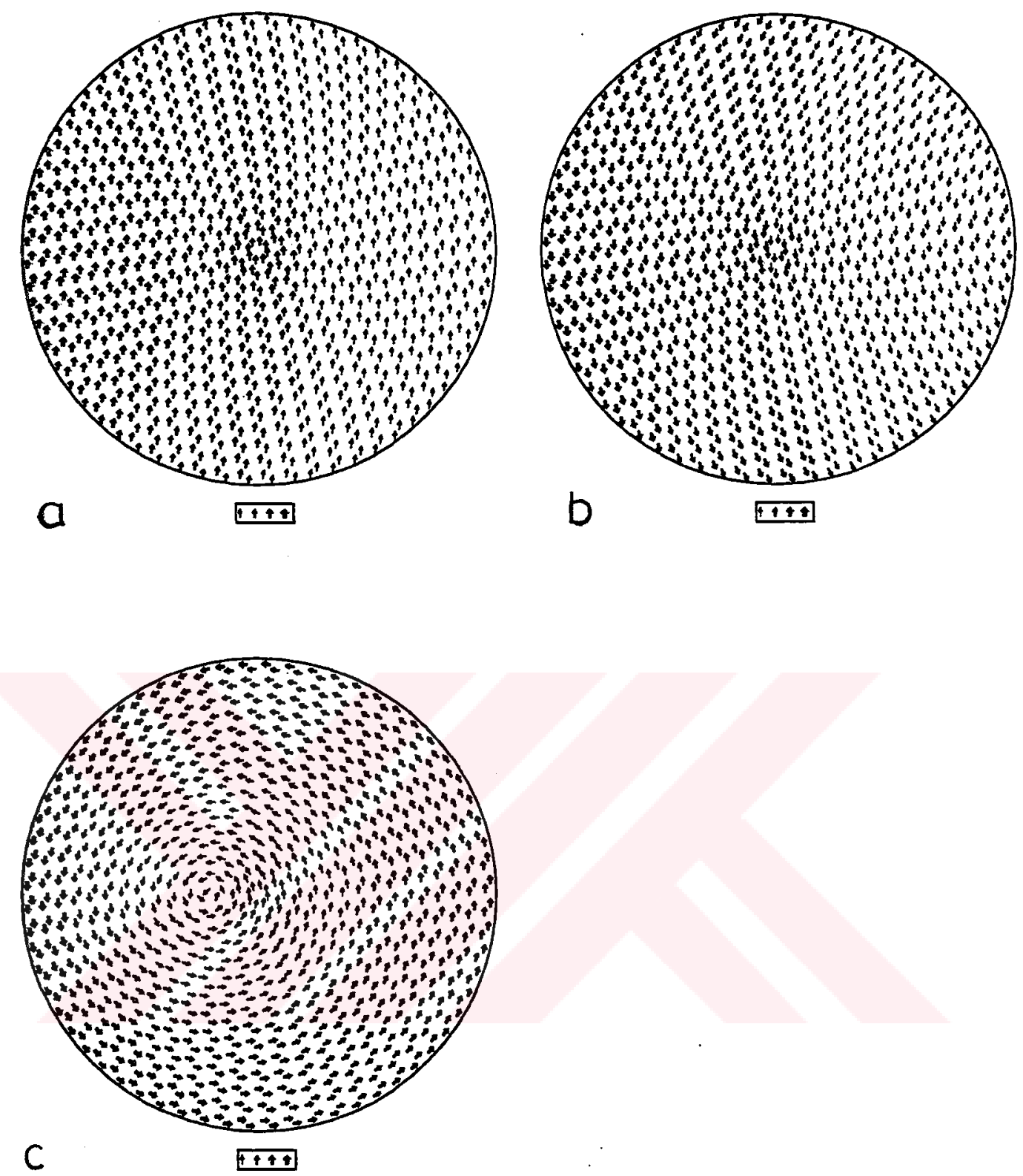

Figure 3.4. Plot of current density distribution and its components for the first coil configuration. A circular coil of radius $0.36 \mathrm{~m}$ is shifted towards positive $x$ axis by $0.23 m$ with respect to the object center. The maximum and minimum magnitudes are also given for each distribution (units are micro Ampere). (a) $-\sigma \nabla \phi$ (maximum $=2.21$, minimum $=1.49)(b)-\sigma \omega \vec{A}(\operatorname{maximum}=4.34$, minimum $=0.64)(\mathrm{c})-\sigma(\nabla \phi+\vec{A})(\operatorname{maximum}=2.13$, minimum $=0.027)$. 

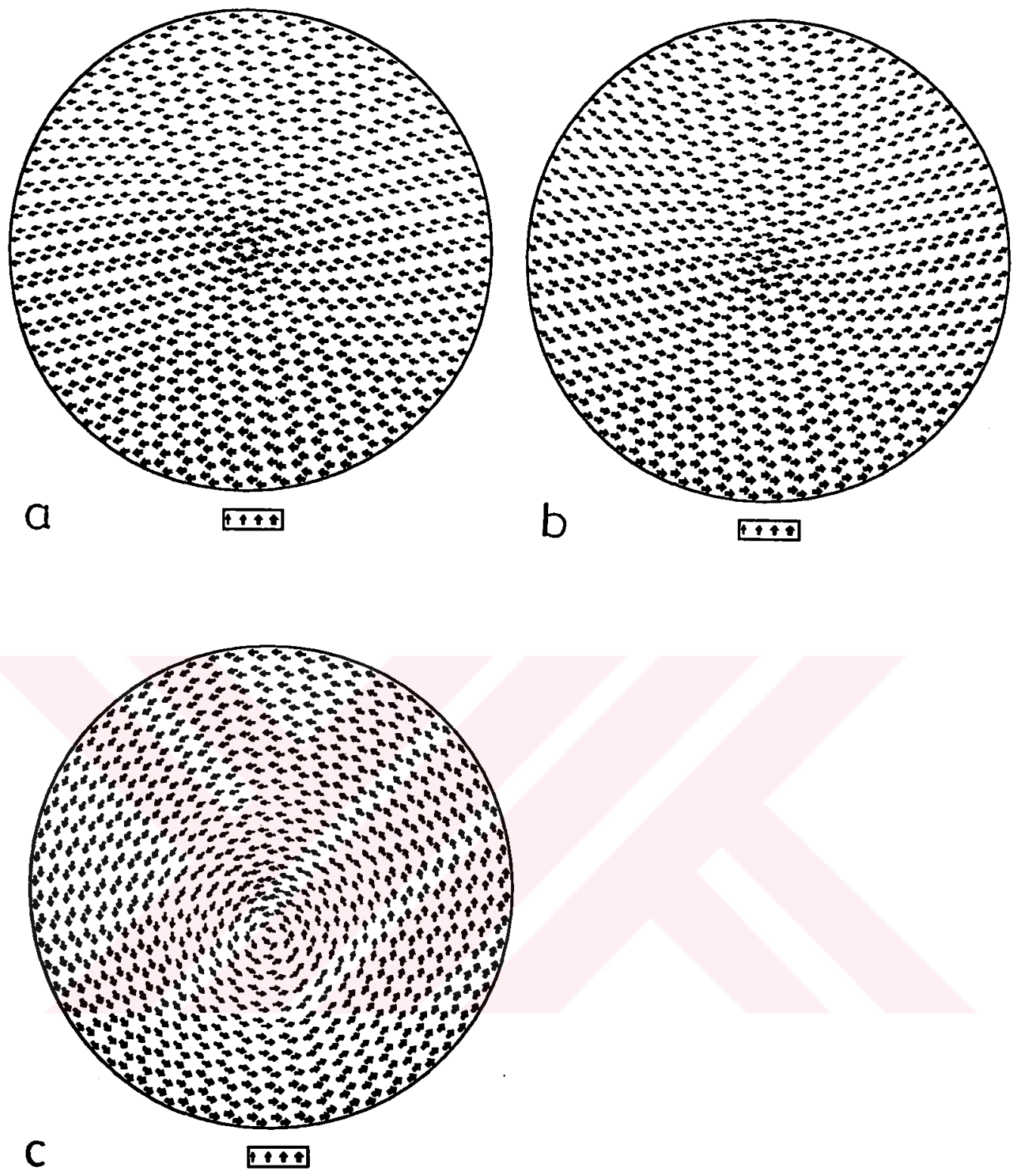

Figure 3.5. Plot of current density distribution and its components for the 2 nd coil configuration. A circular coil of radius $0.36 \mathrm{~m}$ is shifted towards positive $y$ axis by $0.23 m$ with respect to the object center. The maximum and minimum magnitudes are also given for each distribution (units are micro Ampere). (a) $-\sigma \nabla \phi($ maximum $=2.21$, minimum $=1.49)(\mathrm{b})-\sigma \omega \vec{A}(\operatorname{maximum}=4.34$, minimum $=0.64)(\mathrm{c})-\sigma(\nabla \phi+\vec{A})($ maximum $=2.13$, minimum $=0.027)$. 


\subsubsection{Interpreting the sources of perturbations in $\phi$}

In Figure 3.6(a) and (b), the $\nabla(\Delta \phi)$ field, which is equal to $\Delta(\nabla \phi)$, obtained for certain conductivity perturbations are displayed. The coil location is, as it is described in the preceding subsection when the coil is shifted towards $x$ axis with respect to origin. When the conductivity of small rectangular regions are perturbed (these rectangular regions are also shown in these figures), it is evident that the $\nabla \sigma \cdot \vec{E}$ term (expressed in the simpler case of equation 2.26) will yield dipole sources. Indeed, the $\Delta(\nabla \phi)$ field obtained for these conductivity perturbations are as expected from two dipole sources. In Figure 3.6(c), and (d), the corresponding $\Delta(\nabla \phi)$ functions are evaluated on the object boundary and drawn as a function of angular displacement.

The $\Delta(\nabla \phi)$ functions has rich angular variations indicating that the method is sensitive to perturbations in $\sigma$ inside the object. In fact, the sharp peaks in these functions occur at the correct angular positions corresponding to the positions of the conductivity perturbations. If the exciting coil is rotated around the object center, then the exciting $\vec{E}=\nabla \phi_{0}+\vec{A}$ field also changes. For non-uniformities in the central regions the change in the $\vec{E}$ field is primarily in its direction; but, for non-uniformities closer to the object boundary the change in $\vec{E}$ is in its relative magnitude. Both of these changes mean different weighted contributions of non-uniformities to the boundary measurements. 

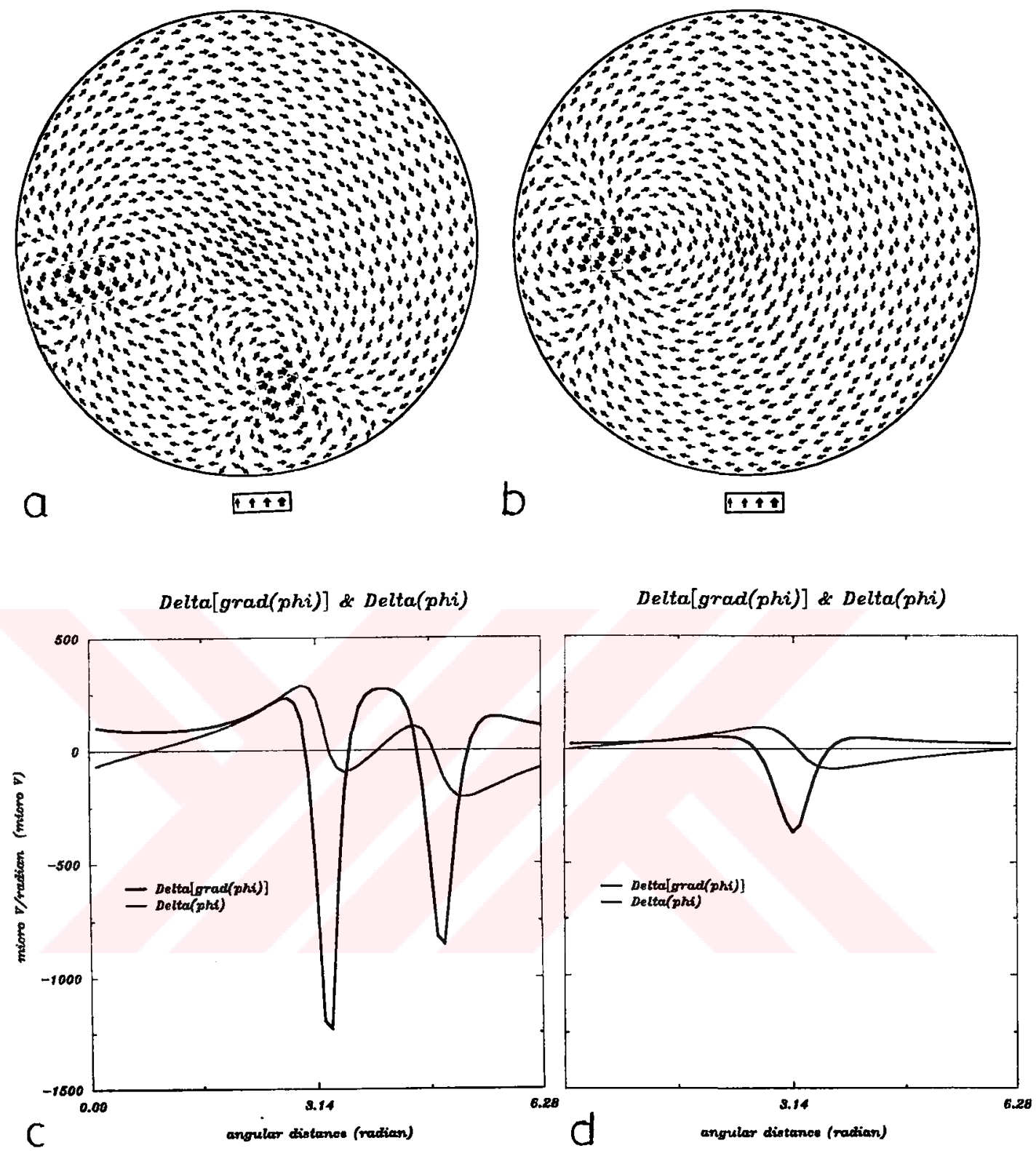

Figure 3.6. (a) $\Delta(\nabla \phi)$ field when two rectangular regions are perturbed to a conductivity of 2 Siemens $/ \mathrm{m}$. The regions are approximately at $180^{\circ}$ and $270^{\circ}$. (b) $\Delta(\nabla \phi)$ field when a rectangular region is perturbed to a conductivity of 2 Siemens $/ \mathrm{m}$. The region is approximately at $180^{\circ}$. (c) and (d) $\Delta(\nabla \phi)$ and $\Delta \phi$ functions evaluated on the object boundary for the perturbations assumed in part (a) and (b), respectively. 


\section{CHAPTER IV}

\section{THE INVERSE PROBLEM}

\subsection{Introduction}

As it is explained in Section 2.4, since in practice, only perturbation in measurements can be obtained for induced current EIT, only the conductivity perturbations compared to a reference can be imaged. In Section 3.2, the problem of imaging conductivity perturbations is readily reduced to provide a solution to a linear system of equations relating perturbation in conductivity to measurement perturbations i.e., $\Delta \bar{G}=R \Delta \bar{\sigma}$ (equation (3.32)). In this equation, $\Delta \bar{G}$ represents $15 M \times 1$ vector of measurement perturbations, $R$ denotes $15 M \times 1016$ sensitivity matrix and $\Delta \bar{\sigma}$ is the $1016 \times 1$ vector of conductivity perturbations (defined on 1016 triangular elements used in FEM formulations). It is clear that, the properties of the $R$ matrix are central to the understanding of the limitations of the induced current EIT.

In this chapter, the number of independent measurements for a specific coil configuration, the sensitivity of measurements to perturbations in pixel conductivities and ill-posedness of the inverse problem are investigated by analyzing the properties of $R$ matrix in detail. Therafter, images reconstructed by simulated data, assuming the use of a specific 9-coil system (with circular coils) are presented. A new coil configuration is suggested to improve the performance of induced current EIT. Using this coil configuration, an approximately linear (spatially) magnetic field distribution is applied on the object which produces relatively large curents in the central region. In this way, sensitivity to objects interior is increased. All coil configurations are compared with respect to singular value patterns and sensitivity distributions. The results are also compared to the performance of injected current EIT. 


\subsection{Properties of the Sensitivity matrix $R$}

In order to understand the effects of coil configuration on the conditioning of the sensitivity matrix and determine the number of independent measurements for a certain coil configuration, the Singular Value Decomposition (SVD) technique is used.

4.2.1 Singular Value Decomposition technique and general matrix properties

The SVD of an $m \times n$ matrix $A(m<n)$ is defined as [36]

$$
A=V \Sigma U^{T}
$$

where the matrix $U$ consists of $m$ orthonormalized eigenvectors $\left(\bar{u}_{i}\right)$ associated with $m$ largest eigenvalues of $A^{T} A$ (the right singular vectors of $A$ ), and the matrix $Y$ consists of $m$ orthonormalized eigenvectors $\left(\bar{v}_{i}\right)$ of $A A^{T}$ (the left singular vectors of $A$ ). $\Sigma$ is the diagonal matrix with diagonal entries equal to the non-zero eigenvalues $\left(\lambda_{1}, \ldots, \lambda_{m}\right)$ of $A^{T} A$ (the singular values of $A$ ) [35], [36]. The singular vectors in $U$ and $V$ are ordered so that the corresponding singular values are decreasing in magnitude. Using the above definitions given for SVD the $A$ and $\bar{c}$ terms in $A \bar{x}=\bar{c}$ equation can be written in series expansion form as

$$
\begin{gathered}
A=\sum_{i=1}^{m} \lambda_{i} \bar{v}_{i} \bar{u}_{i}^{T} \\
\bar{c}=\sum_{i=1}^{m} \lambda_{i}\left(\bar{u}_{i}^{T} \bar{x}\right) \bar{v}_{i}
\end{gathered}
$$

The notion of condition can be extended to general rectangular matrices [36], and the condition number of $A(\kappa(A))$ can be defined as

$$
\kappa(A)=\frac{\lambda_{1}}{\lambda_{m}}
$$

It is known that $\kappa(A)$ quantifies the sensitivity of $A \bar{x}=\bar{c}$ problem to the noise in the measurement vector $\bar{c}$ (relative error in $\bar{x}$ is less than or equal to $\kappa(A)$ times the relative error in $\bar{c}$ ) [36]. If $\kappa(A)$ is large then $A$ is said to be ill-conditioned whereas a small condition number will show that it is a well-conditioned matrix. 


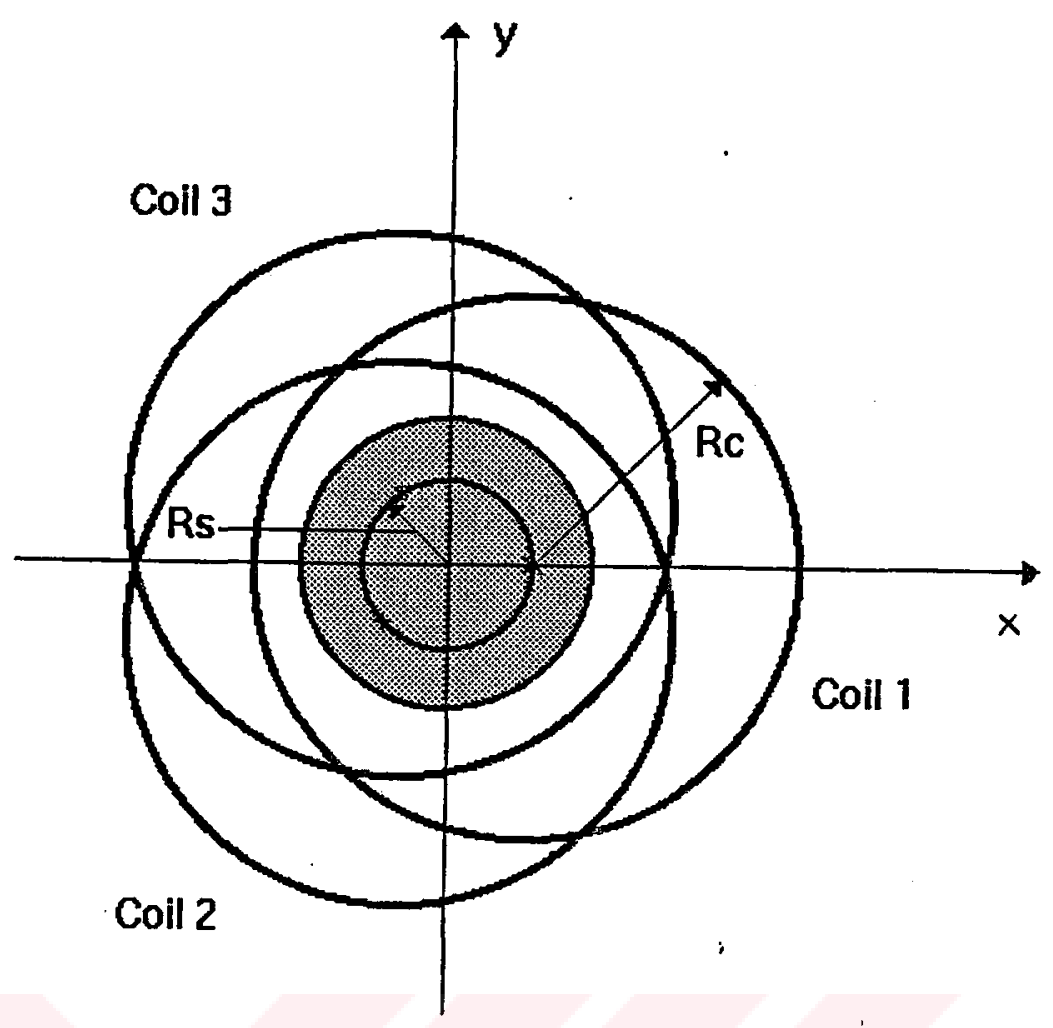

Figure 4.1. Placement of 3 circular coils on the plane of a circular object. The coils have equal radii $\left(R_{c}\right)$ and their centers (shown by $\times$ marks) are placed on a circle of radius $R_{s}$ with equal angular seperations.

\subsubsection{Effects of coil configuration on $\kappa(R)$}

In this study, the effects of coil configuration on the conditioning of sensitivity matrix is analyzed by using 3 circular coils $(M=3)$ of equal radii $\left(R_{c}\right)$ which are placed around a circular object (radius $=0.12 m$ ). The centers $\rightarrow$ of the coils are located with equal angular sepgrations on a circle of radius $R_{s}$ concentric with the circular object (see Figure 4.1). In the first part of the study, the coil radii are chosen to be $0.36 m$. The radius $\left(R_{s}\right)$ of the circle on which the coil centers are located is increased five times from $0.04 \mathrm{~m}$ to $0.20 \mathrm{~m}$ by $0.04 \mathrm{~m}$ steps. For each case, the sensitivity matrix is calculated and corresponding singular value pattern is obtained by SVD (Figure 4.2). Note that, for a fixed coil radius, as $R_{s}$ increases the object gets closer to specific coil portions (to a certain point on each coil). It is observed (from Figure 4.2) that, as the distance between an object side and an off-centered coil minimizes, the magnitude of the singular values $\lambda_{k}(k>15)$ increase with respect to the first 15 singular values 
(leading small condition numbers). This result is actually expected, since, for the worst case when $R_{s}=0$ (which is not shown in Figure 4.2), the three coils will be on top of each other and there can only be 15 independent measurements.

In the second part of this study, assuming the minimum distance between each coil and object to be $0.01 \mathrm{~m}$, the radius of coils is increased five times from $0.15 \mathrm{~m}$ to $0.36 \mathrm{~m}$. Again, for each coil configuration the sensitivity matrix is calculated and singular value pattern is obtained using SVD. The resultant five singular value patterns are given in Figure 4.3. It is observed that when the minimum distance between coils and object is kept fixed, the sensitivity matrix has a better singular value decay pattern (and small condition numbers) when one uses coils of larger radii. However, after some $R_{c}$ value (in this case $R_{c}=0.36 \mathrm{~m}$ ), using coils of larger radii does not improve the decay pattern of singular values, i.e., far side of larger coils becomes ineffective in current induction. The worst case, which results in only 15 nonzero singular values can occur if coils of radii $0.13 m(0.12 m+0.01 m)$ are used (where again coils will be on top of each other). Therefore, if circular coils is to be used in induced current EIT, it is better to use coils which have sufficiently large radii and locate them as close as possible to an object side in order to obtain better conditioned sensitivity matrices.

\subsubsection{Number of independent measurements for an $N$-electrode system}

It is known that, for an $N$ electrode system, one can obtain a maximum $N(N-1) / 2$ independent measurements in EIT using injected currents [11], [46], [34]. The purpose of this section is to understand whether it is possible, using current induction method with circular coils, to obtain more independent measurements using $N$ electrodes.

For a specific case, when 16 electrodes are attached to the object surface, it necessary to show that one can obtain more than 120 independent measurements by using certain number of circular coils. In order to understand this fact, 9 circular coils of equal radii $\left(R_{c}=0.36 \mathrm{~m}\right)$ are assumed to be placed 
Singular Value Plots for different coil locations

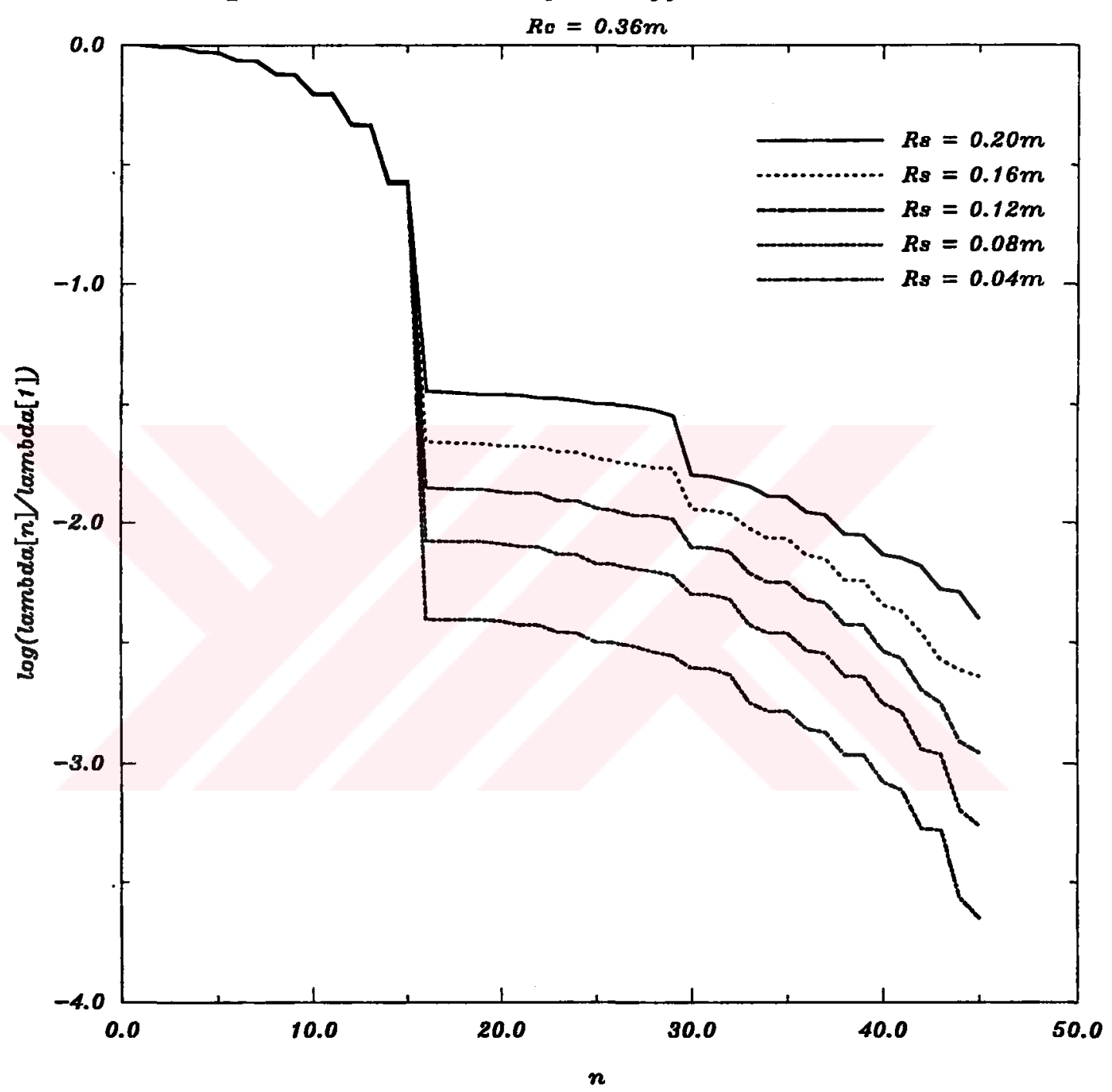

Figure 4.2. Singular value patterns of sensitivity matrices obtained for the 3-coil system when the coil center shifts $\left(R_{s}\right)$ are assumed to be $0.04 m, 0.08 m$, $0.12 \mathrm{~m}, 0.16 \mathrm{~m}$ and $0.20 \mathrm{~m}$. For each coil configuration, the singular values are normalized with respect to the maximum singular value. 
Singular Value Plots due to change in coil radii

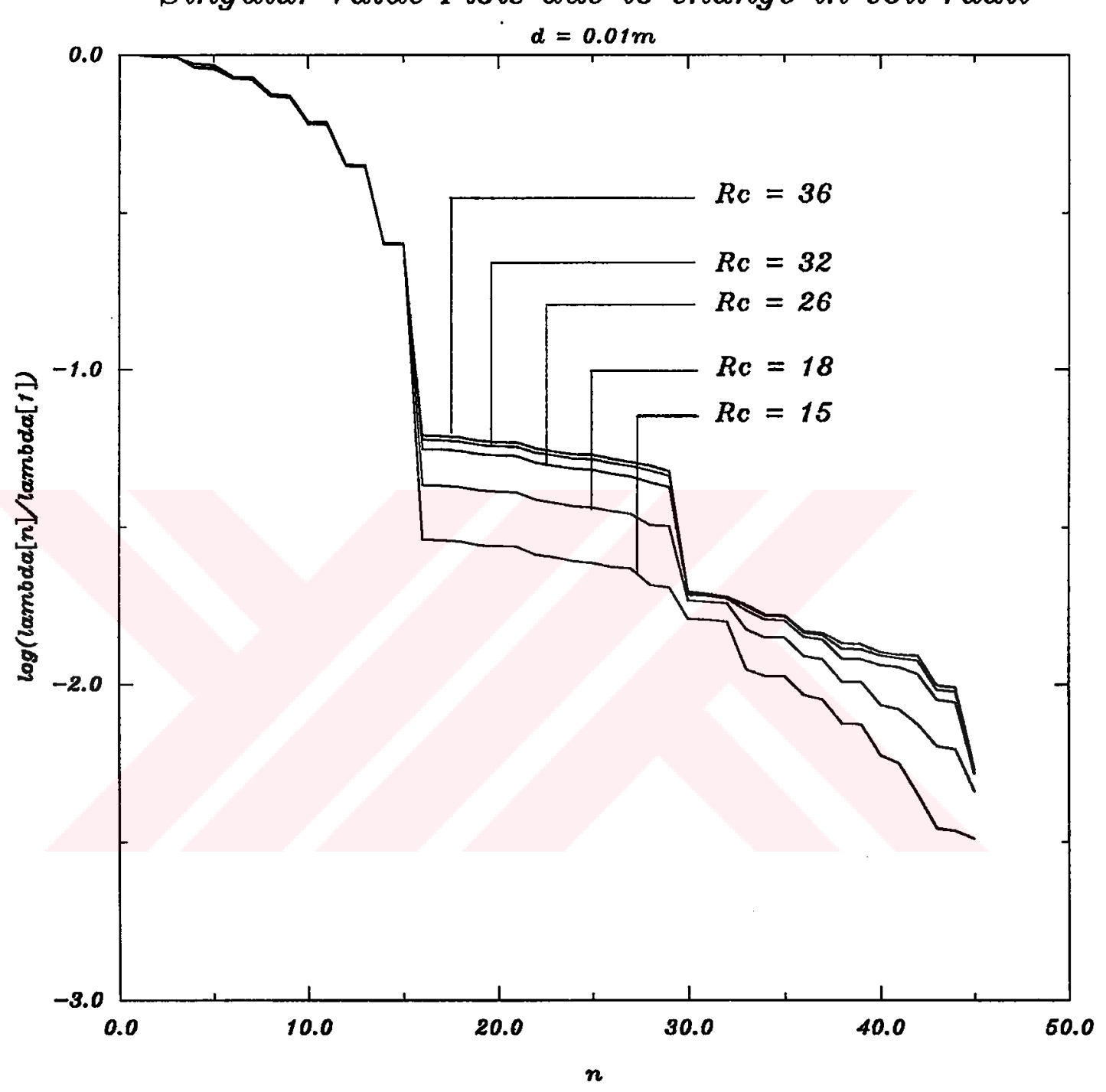

Figure 4.3. Singular value patterns of sensitivity matrices obtained for the 3-coil system. The radius of coils $\left(R_{c}\right)$ are assumed to be $0.15 m, 0.18 m, 0.26 m$, $0.32 m$ and $0.36 m$ in each case. For each coil configuration, the singular values are normalized with respect to the maximum singular value. 
around the circular object. The centers of the coils are located on a circle of radius $0.23 m$ with equal angular seperations (a simple extention of the coil configuration used previously). In order to determine the number of independent measurements in this system, the singular values of $R$ is obtained by SVD. Since the number of measurements are less than the number of unknowns, any dependent measurement will yield a zero singular value and therefore one can determine the number of independent measurements by looking at the number of nonzero singular values. If for each excitation there exist a maximum of 15 independent measurements, the number of nonzero singular values should be maximally $135(=15 \times 9)$. The sensitivity matrix and corresponding singular values are calculated and the resultant normalized singular value pattern is given in Figure 4.4. It is observed that there are indeed 135 nonzero singular values. In order to compare the singular value patterns of the induced and injected current EIT methods, the sensitivity matrix for injected current EIT (using Sheffield data collection protocol) is also calculated using a similar approach to the one used in this study for induced current EIT (details are presented in [20]). In injected current EIT, using the Sheffield 'adjacentdrive adjacent-measurement' data collection protocol, the number of independent measurements is 104 when 16 electrodes are used [10] (For an N electrode injected current EIT system, a maximum of $\mathrm{N}(\mathrm{N}-3) / 2$ independent measurements are obtained taking care of not collecting data using current carrying electrodes because of contact impedance problem). The resultant normalized singular value decay pattern (which is very similar to the ones computed in [8]) are also given in Figure 4.4. It is observed that after the $56^{\text {th }}$ singular value, the singular values of induced current EIT are much larger than the ones obtained for injected current EIT. The ratio of maximum singular value to the $104^{\text {th }}$ singular value is 584656 in injected current EIT whereas it is 4083 in induced current EIT. The ratio of maximum to minimum singular value is 121814 in the induced current system. It can be concluded that induced current EIT is better conditioned than injected current EIT and it provides more independent measurements compared to the injected current case 


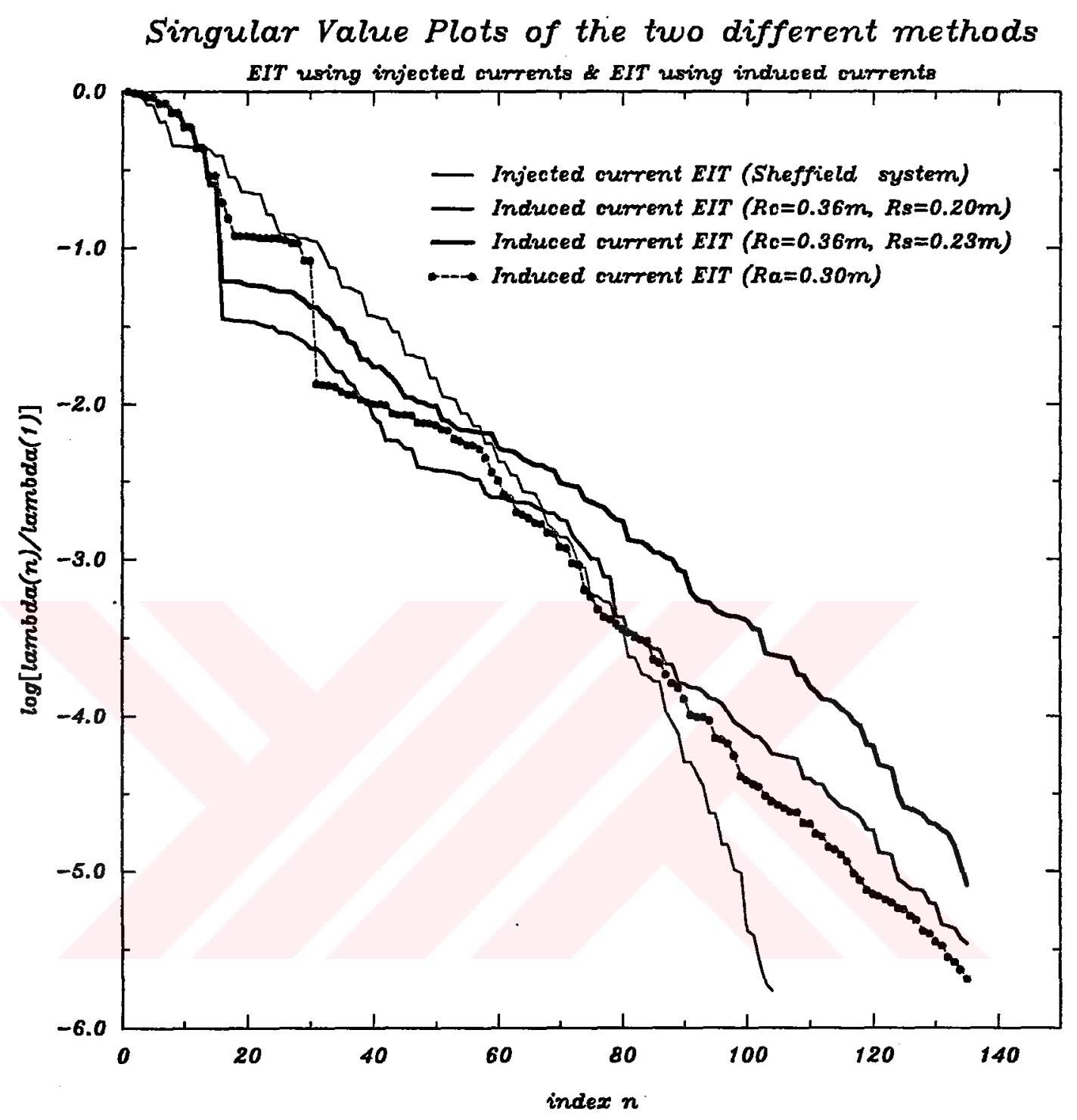

Figure 4.4. Singular value patterns of induced and injected current EIT. 9 circular coils of equal radii $(0.36 \mathrm{~m})$ are assumed to be placed around the circular object (radius $=0.12 m$ ). The centers of coils are on the circle (concentric with the object origin) of radius $0.20 \mathrm{~m}$ (and $0.23 \mathrm{~m}$ ). The singular values for the case of an alternative coil configuration are also given. The singular value pattern of injected current EIT is obtained assuming that Sheffield data collection protocol is used. For all EIT systems, the singular values are normalized with respect to their own maximum singular values and plotted on the same graph. 


\subsubsection{Sensitivity analysis}

By displaying the rows of $R$ it is possible to visualize the sensitivity of any potential difference measurement to pixel conductivity perturbations. In Figure 4.5 , the $8^{\text {th }}, 10^{\text {th }}, 12^{\text {th }}$ and $14^{\text {th }}$ rows of the sensitivity matrix (calculated for a coil of radius $0.36 \mathrm{~m}$ and shifted to the right $0.23 \mathrm{~m}$ along the $x$ axis from the origin of $\Omega$ ) are diplayed. It is observed that the measurement is much more sensitive to a conductivity perturbation near the measuring electrodes, and furthermore it is least sensitive to the central region.

In order to visualize the overall sensitivity pattern as compared to the displays of single rows of $R$, the concept of total lead sensitivity is used. This concept is originally proposed by Eyuboglu in [17] for the comparison of the two injected current EIT systems (i.e, polar-drive versus adjacent-drive). In this method, the entries of a vector $\bar{T}$, which is named as the total lead sensitivity vector are calculated as follows

$$
T_{j}=\sum_{i=1}^{15 M}\left|R_{i j}\right|
$$

where $\mathrm{j}=1 \ldots$ 1016. As its name implies, an entry of $\bar{T}$ is a measure of the overall sensitivity of the measurements to the conductivity perturbation of the corresponding element. This vector is displayed in Figure 4.5(a). It can be clearly seen that conductivity perturbation in the central region effect the measurements less.

The $\bar{T}$ displayed in Fig 4.6(a) is circularly symmetric due to the symmetry in excitations and measurements in the 9-coil system. $\bar{T}$ for injected current EIT (displayed in Figure 4.6(b)) also exhibits a circularly symmetric distribution [15]. Hence, the comparison of the two methods using the total lead sensitivities can be made by plotting the average sensitivity values on a radial line (Actually the average is taken over an angular displacement of $2 \pi$ for a given radial distance). The normalized values (with respect to the minimum) of each system are plotted (Figure 4.7). It is observed that both the injected and induced current EIT systems are mostly sensitive to the conductivity perturbations occuring nearby the object boundary. The injected current EIT system 
is more sensitive to the inner region conductivity perturbations compared to induced current EIT system for the particular 9-coil configuration used.

\subsection{Image Reconstruction}

Assuming the use of 9 coils $(M=9)$ for current induction, the sensitivity matrix $R$ becomes a $135 \times 1016$ matrix. Therefore, for this particular coil configuration, equation (3.32) represents an underdetermined system. An underdetermined system either has no solution (if $\Delta \bar{G}$ is not in the range space of $R$ ) or has an infinity of solutions. In the case of noiseless data, the SVD technique can be used to compute the minimal norm solution of $\Delta \bar{G}=R \Delta \bar{\sigma}$ problem:

$$
\Delta \bar{\sigma}=\sum_{i=1}^{135} \frac{1}{\lambda_{i}}\left(\overline{v_{i}^{T}} \Delta \bar{G}\right) \overline{u_{i}}
$$

However, in the case of noisy data $(\Delta \bar{G} \rightarrow \Delta \bar{G}+\bar{n})$, the solution of $\Delta \bar{\sigma}$ given in the above form (including all 135 terms) will yield erroneous results since $\Delta \bar{\sigma}$ will now be expressed as

$$
\Delta \bar{\sigma}=\sum_{i=1}^{135}(\underbrace{\frac{1}{\lambda_{i}}\left(\bar{v}_{i}^{T} \Delta \bar{G}\right) \bar{u}_{i}}_{\text {signal term }}+\underbrace{\left.\frac{1}{\lambda_{i}}\left(\bar{v}_{i}^{T} \bar{n}\right) \bar{u}_{i}\right)}_{\text {noise term }}
$$

Truncating the series at some index $(i=k)$ is necessary in order to avoid the effective noise contributions. Mathematically, for all the terms that should be truncated the following inequality is valid.

$$
\frac{1}{\lambda_{k}} \bar{v}_{k}^{T} \bar{n} \geq \frac{1}{\lambda_{1}} \bar{v}_{1}^{T} \Delta \bar{G}
$$

If $\|\cdot\|$ denotes the 2-norm [36], the above equation can be transformed into the following one

$$
\frac{1}{\lambda_{k}}\left\|\overline{v_{k}}\right\|\|\bar{n}\| \geq \frac{1}{\lambda_{1}}\left\|\overline{v_{1}}\right\|\|\Delta \bar{G}\|
$$

Since $\bar{v}_{i}(i=1 \cdots 135)$ is an orthonormal vector (i.e $\left.\left\|\bar{v}_{i}\right\|=1\right)$ this property yields

$$
\frac{1}{\lambda_{k}}\|\bar{n}\| \geq \frac{1}{\lambda_{1}}\|\Delta \bar{G}\|
$$



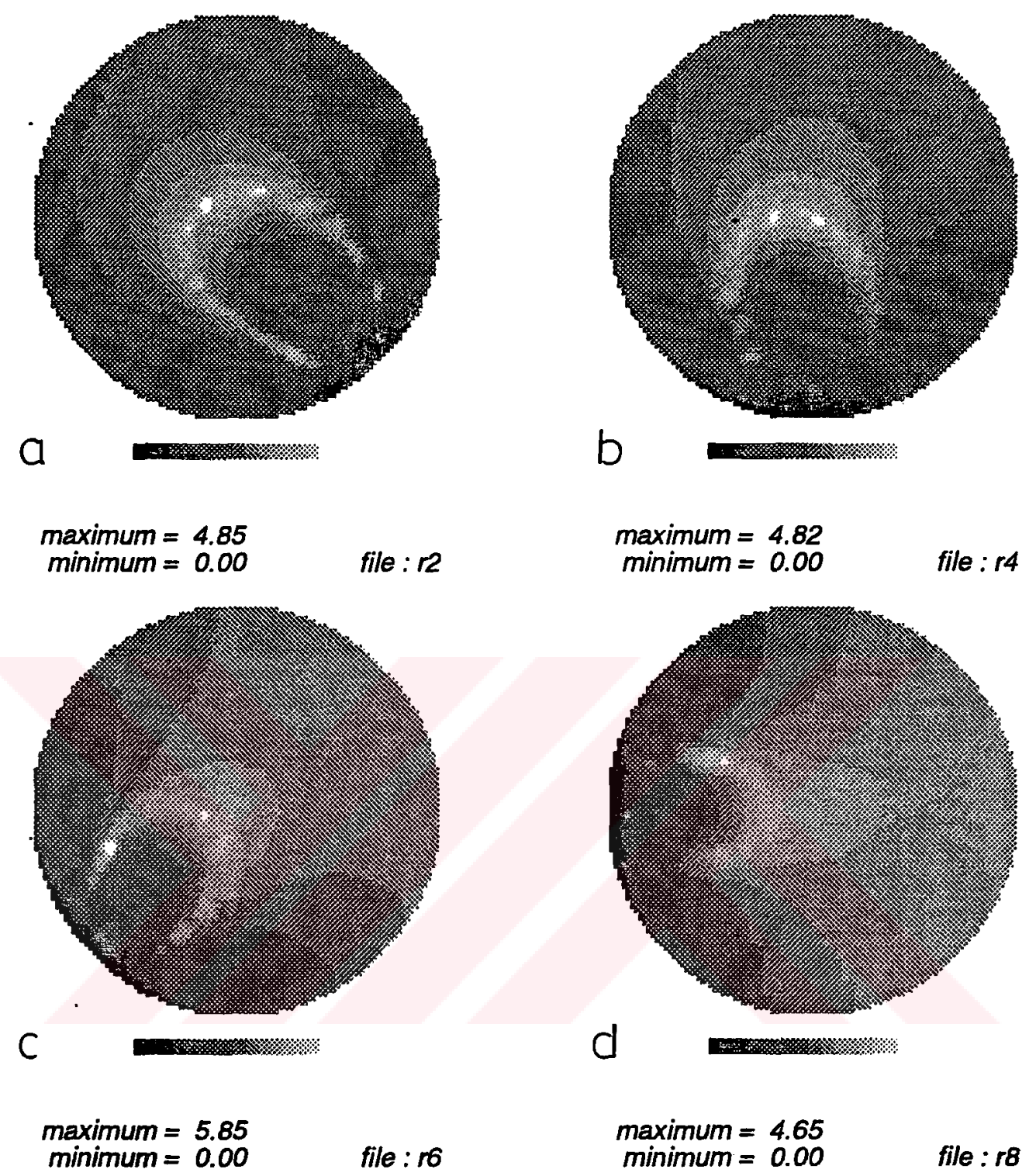

file : r6

file : $r 8$

Figure 4.5. Sensitivity patterns of measurements to conductivity perturbations. A circular coil of radius $0.36 \mathrm{~m}$ is placed off-centered by $0.23 \mathrm{~m}$ towards positive $x$ axis with respect to object origin. The logarithm of the normalized values (with respect to minimum) are displayed. (a) Sensitivity of $8^{\text {th }}$ measurement to pixel conductivity perturbations. (b) Sensitivity of $10^{\text {th }}$ measurement to pixel conductivity perturbations. (c) Sensitivity of the $12^{\text {th }}$ measurement to pixel conductivity perturbations. (d) Sensitivity of $14^{\text {th }}$ measurement to pixel conductivity perturbations. 


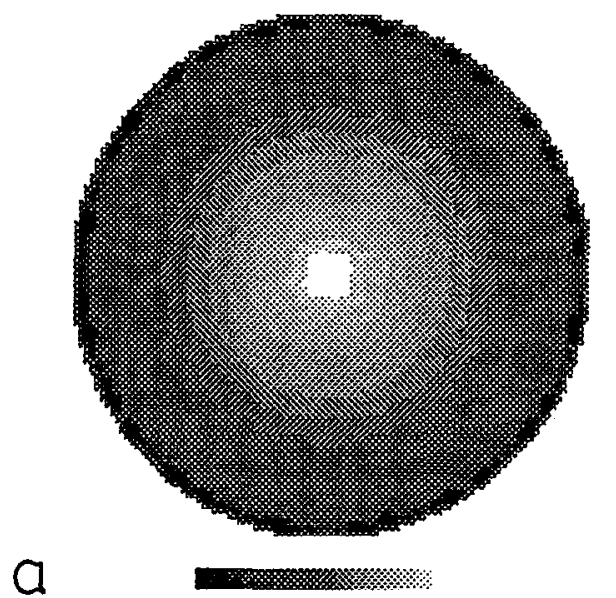

$$
\text { maximum }=2.17
$$
minimum $=0.00$

$$
\text { file : totld1.ind }
$$

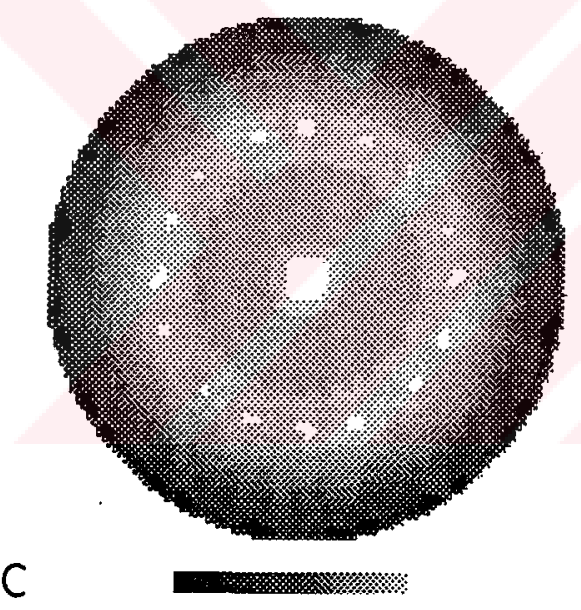

$$
\text { maximum }=1.48
$$

minimum $=0.00$

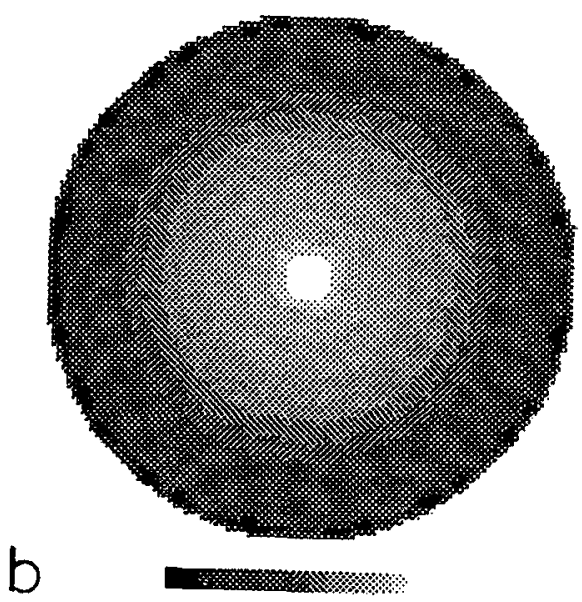

maximum $=1.56$

file : totld1.inj

Figure 4.6. Display of total lead sensitivity vectors of induced and injected current EIT. The logarithm of the normalized values (with respect to minimum) are displayed. (a) Total lead sensitivity for induced current EIT using 9 circular coils. (b) Total lead sensitivity of injected current EIT with Sheffield Data collection protocol. (c) Total lead sensitivity for induced current EIT using an alternative coil configuration. 
Comparison of the Total Lead Sensitivies

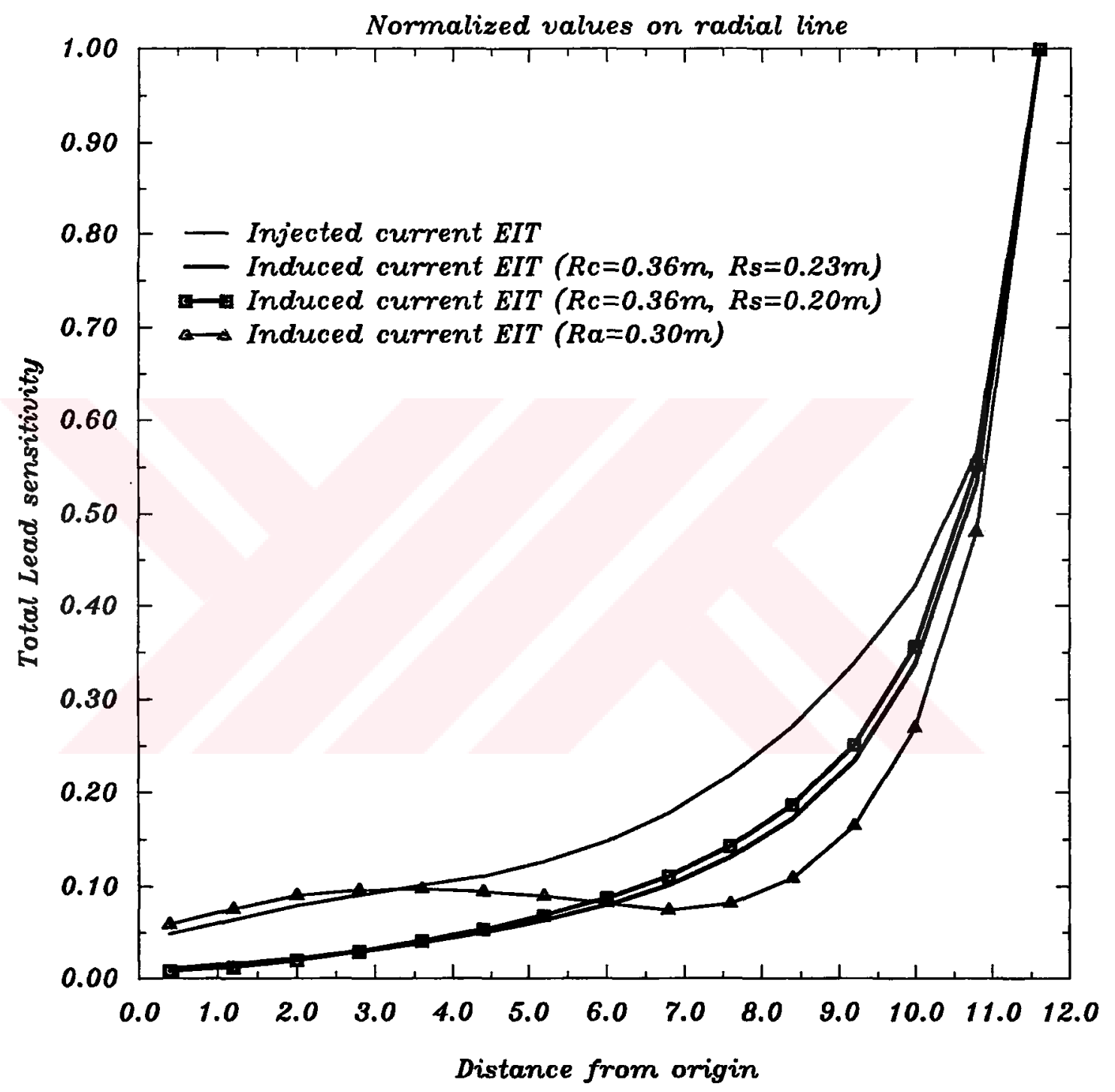

Figure 4.7. Average sensitivity values of injected and induced current EIT systems evaluated on a radial line using corresponding total lead sensitivity vectors 


$$
\lambda_{k} \leq \frac{1}{\frac{\|\Delta \bar{G}\|}{\|\bar{n}\|}} \lambda_{1}
$$

or

$$
\lambda_{k} \leq \frac{\lambda_{1}}{S N R}
$$

Taking the SNR of a practical system to be $80 \mathrm{~dB}[66],[70],[77],[61]$, the singular values smaller than the $0.1 \%$ of the maximum singular value are not used in the computation of $\Delta \bar{\sigma}$ (which results in the use of 88 singular values). Using the data obtained for the simulation distribution given in Figure 4.8(a) a conductivity distribution is reconstructed and is given in Figure $4.8(\mathrm{~b})$. The image of the same conductivity distribution is also reconstructed by injected current EIT using a similar approach and given in Figure 4.8(c) [20]. It is observed that, the image reconstructed by induced current EIT is indicative of the position and size of the inhomogeneity inside the object and, in this respect, it is comparable to the image obtained by injected current EIT.

\subsection{An alternative coil configuration}

The importance of the coil configuration on the properties of $R$ matrix, such as decay pattern of the singular values, condition number and sensitivity of measurements to inner regions have been shown using even the 9-coil system in the preceeding sections. In this section, an alternative coil configuration is suggested in order to improve the above given parameters of an induced current EIT system.

Figure 4.9 shows the basic principles of excitation using this alternative coil configuration. Since the magnetic field density change sign on the coil axis, the induced currents are expected to flow in counter-clockwise and clockwise directions on the left and right sides of the object region respectively. As a result, currents in the central region will be strengthened. The current density distribution and its separate components are calculated for a specific coil location and given in Figure 4.10. It is observed from Figure 4.10(c) that, the currents induced in the central region is relatively increased, however, there are still larger currents at the left and right edges of the object. The smalles currents are localized between the center and these edges. 


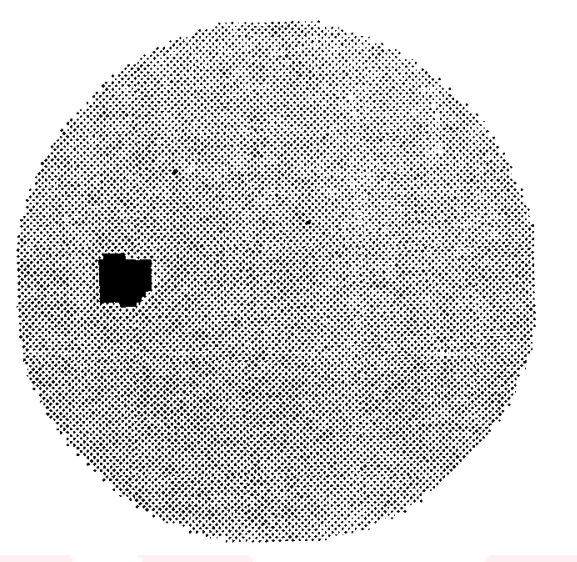

a

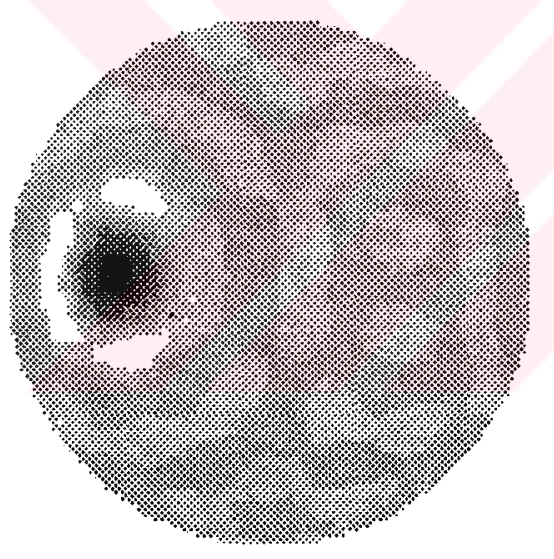

C

Figure 4.8. Comparison of reconstructed images obtained by induced and injected current EIT. (a) The simulated conductivity distribution. The conductivity of inhomogeneity (shown by black) is 2 Siemens $/ m$ whereas the background conductivity is 0.2 Siemens $/ m$. (b) Reconstructed image of $\Delta \bar{\sigma}$ using induced current EIT, maximum $=0.160$ Siemens $/ \mathrm{m}$, minimum $=-0.053$ Siemens $/ \mathrm{m}$. (c) Reconstructed image of $\Delta \bar{\sigma}$ using injected current EIT, maximum $=$ 0.250 Siemens $/ m$, minimum $=-0.037$ Siemens $/ m$. (d) Reconstructed image of $\Delta \bar{\sigma}$ using induced current EIT with alternative coil configuration, maximum $=0.150$ Siemens $/ m$, minimum $=0.060$ Siemens $/ m$. 


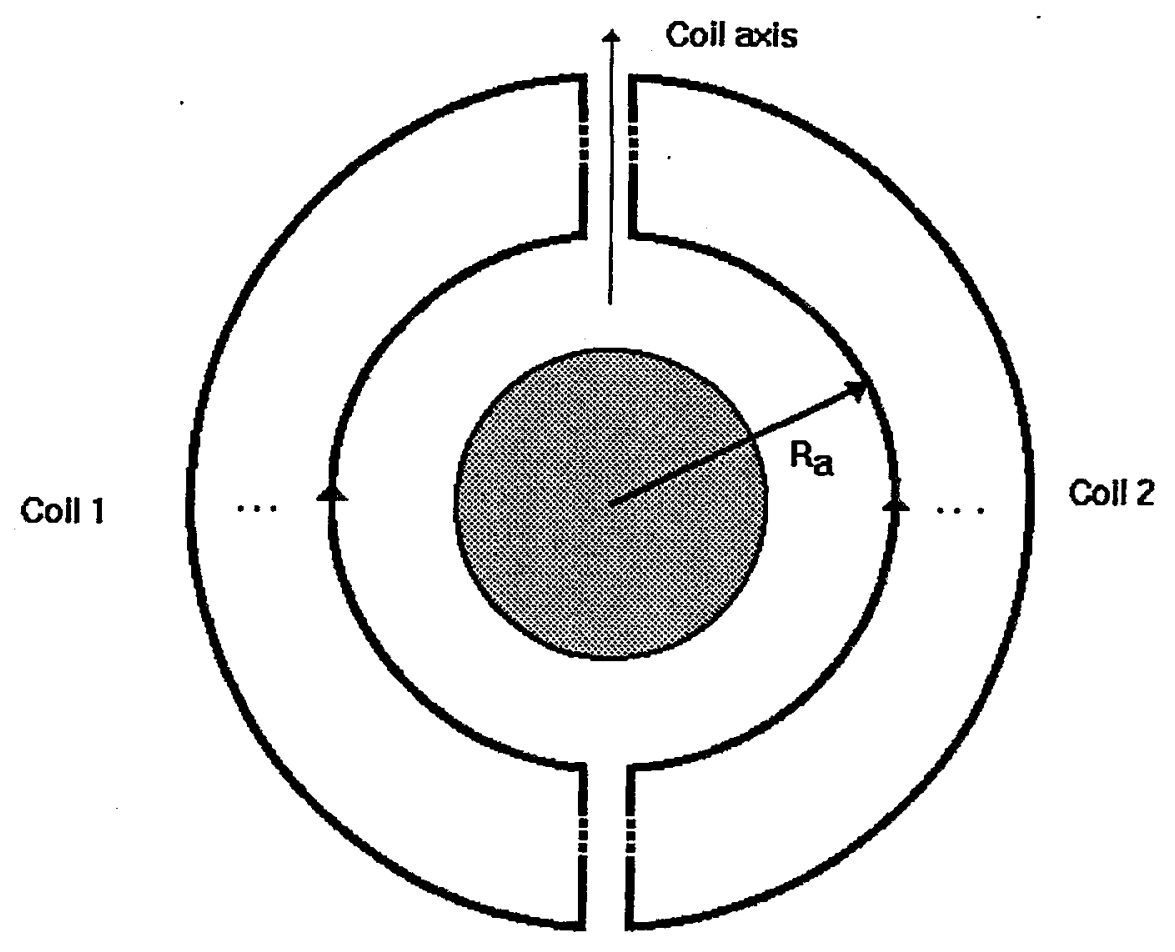

Figure 4.9. An alternative coil configuration. $R_{a}$ denotes the radius of arcs.

By rotating this coil configuration or placing similar coils around the object with different orientations (so that coil axes are towards different directions) one may induce different current distributions inside the object and hence collect different data sets from the surface. In this study, the arc distances from the object origin are chosen to be $0.30 \mathrm{~m} .9$ coils are assumed to be placed so that the coil axes show 9 different directions with equal angular separations.

The sensitivity matrix is calculated for the above given coil configuration and singular values are obtained using SVD. The resultant singular value pattern is shown in Figure 4.4 for comparing its decay behaviour with respect to induced current EIT with 9 circular coils and injected current EIT. It is observed that, the sensitivity matrix obtained for this coil configuration is again better conditioned then injected current EIT, furthermore, there is now large 30 singular values before the first noticable jump occurs. The total lead sensitivity vector is displayed in Figure 4.6 (c)and the average sensitivity values on a radial line are given in Figure 4.7. It is observed that, the sensitivity of measurements to inner regions is increased (even better than injected current 

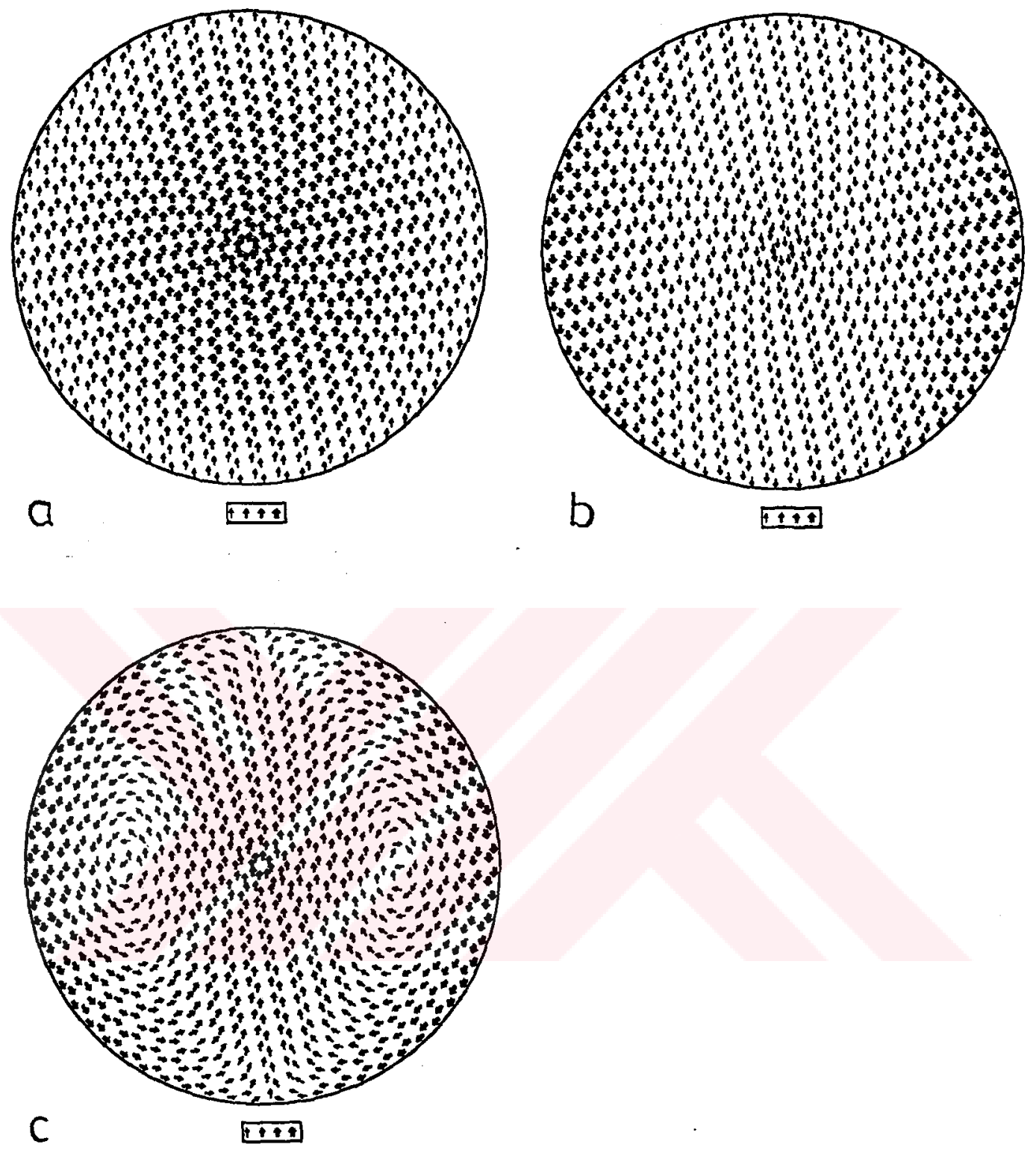

Figure 4.10. Induced current density distribution and its components when alternative coil configuration is used. The conductivity distribution is uniform (0.2Siemens $/ \mathrm{m}) . R_{a}=0.30 \mathrm{~m}$. (units are micro Ampere) (a) $-\sigma \nabla \phi$, maximum $=2.60$, minimum $=2.50,(\mathrm{~b})-\sigma \omega \vec{A}$, maximum $=2.70$, minimum $=2.50$, (c) $-\sigma(\nabla \phi+\omega \vec{A})$, maximum $=0.20$, minimum $=0.0037$ 
EIT). The sensitivity of conductivity perturbations on a ring-like path (where the smallest currents are localized) is least. The reconstructed image of the simulated conductivity distribution (Figure 4.8 (a)) is also given in Figure 4.8 (d). 


\section{CHAPTER V}

\section{DESIGN OF A PROTOTYPE DATA ACQUSITION SYSTEM}

\subsection{Introduction}

This chapter includes the studies performed for the realization of a data acqusition system for EIT using induced currents. The aim was to verify the formulation and solution methods developed for the solution of the forward and inverse problems by experimental studies. Since the system was planned to be a prototype, the design, construction, data collection and image reconstruction phases were completed in the shortest period of time. It was not designed to fulfill certain specifications like high accuracy in the measurements and short data collection time. Most of the blocks in the system probably need to be redesigned for more accurate and fast data collection in the possible future studies. However, in spite of its limited mesurement specifications, this prototype gave very promising initial results. It provided an opportunity to understand the validity of formulations and assumptions by using real data and made it possible to test the adequecy of the numeric models.

In this chapter, following a system overview section, the different blocks of the system are presented with necessary circuit diagrams and explanations. Thereafter, experimental results are given using real data obtained from 2-D object distributions. The measurements are compared with the calculated ones obtained by computer simulations and then images reconstructed by using SVD are presented. 


\section{System Overview}

The block diagram of the prototype is given in Figure 5.1. The system is basically made up of two separate parts, i.e coil current drive system and voltage measurement system. The coil current drive system includes a $50 \mathrm{kHz}$ sine wave generator, a power amplifier capable of providing $0-2 \mathrm{~A}$ current, two computer controlled relay boards to select any one of 16 coils and a number of circular coils all of which are tuned to $50 \mathrm{kHz}$ by a single series capacitance. The voltage measurement system includes buffers, measurement electrode selection multiplexers, an instrumentation amplifier, a phase sensitive detection circuit for quadrature component of the measurement, a low pass filter and a variable gain amplifier. The drive coil and measurement electrode pair selection is made by software controlled Techmar LabMaster data acqusition card installed in a PC-XT. The LabMaster provides 24 digital lines via a 8255 parallel programmable interface, a 12 bit $A / D$ conversion facility with $30 \mathrm{kHz}$ sampling frequency and a means of 12 bit $\mathrm{D} / \mathrm{A}$ conversion. The 24 digital lines are buffered and isolated from PC by opto-couplers. $8(2 \times 4)$ of the lines are used for the selection of voltage measerement electrodes and 4 of them are used for the selection of drive coils.

\subsection{Circuit Descriptions}

The first block of the current drive system is the power amplifier (Figure 5.2) which is designed and constructed by Haldun Ozdemir. It is capable of driving currents up to $2 \mathrm{~A}$ at $50 \mathrm{KHz}$. The amplitude of the currents is controlled by the input voltage signal applied by a suitable signal generator and monitored by observing the voltage on a power resistor named as $R_{\text {monitor }}$. The current fed by the power amplifier is multiplexed to one of the coils placed around a 2-D phantom (Figure 5.3(a)). The multiplexing circuit is made up of two same boards one of which is mounted on top of an other. Each board includes a $16 \times 1$ analog multiplexer/demultiplexer (HEF 4067) which has a DC $+5 \mathrm{~V}$ in its input. The 16 output channels are connected to 16 relay drive circuits as shown in Figure 5.3(b). Depending on the select word sent by PC, one of these relays are driven at the same time on each board, together connecting 


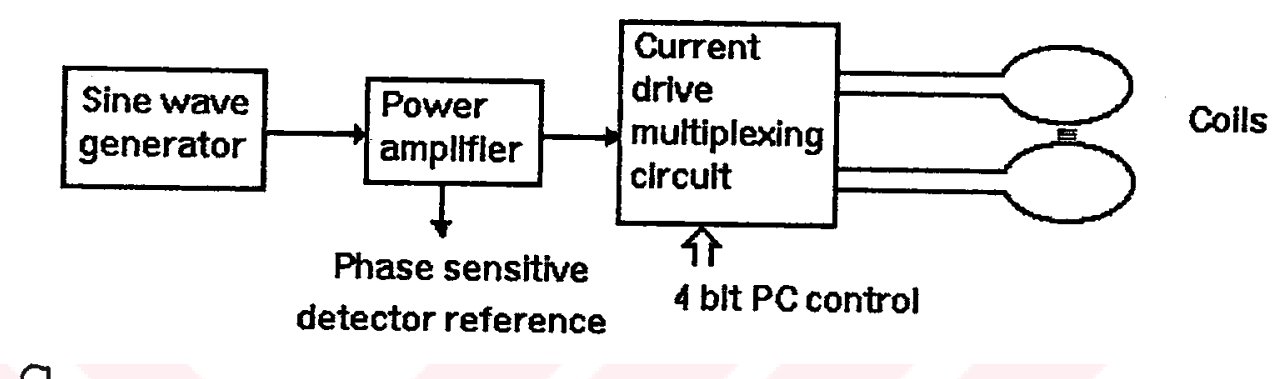

$a$

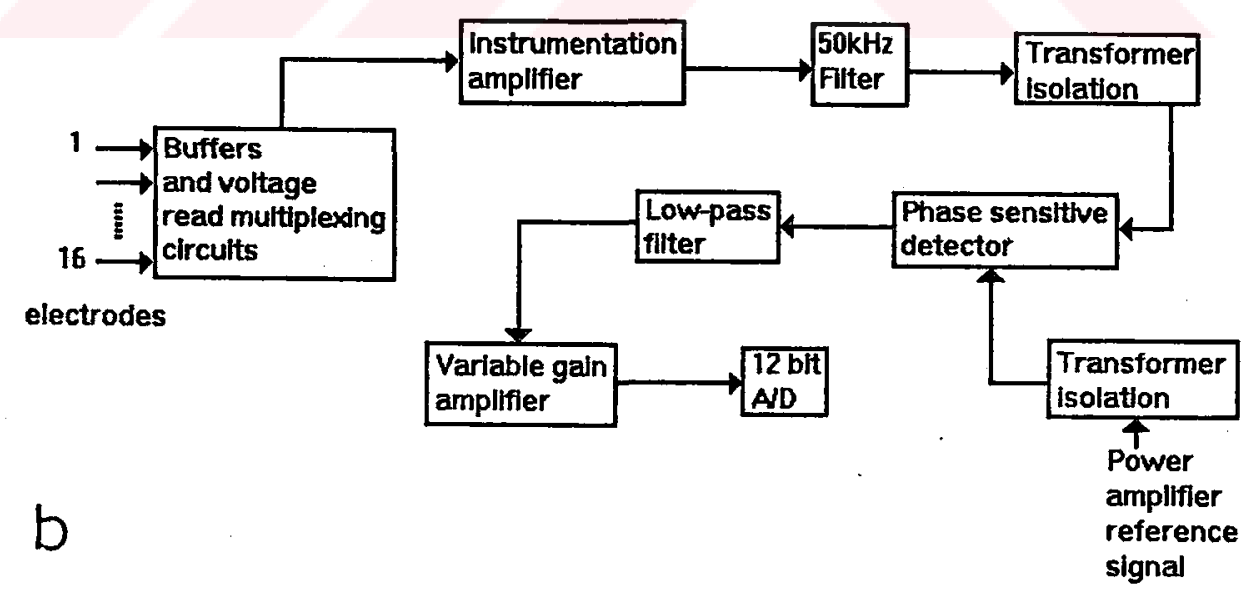

Figure 5.1. Block diagram of the prototype. (a) Coil current drive system, (b) Voltage difference measurement system 


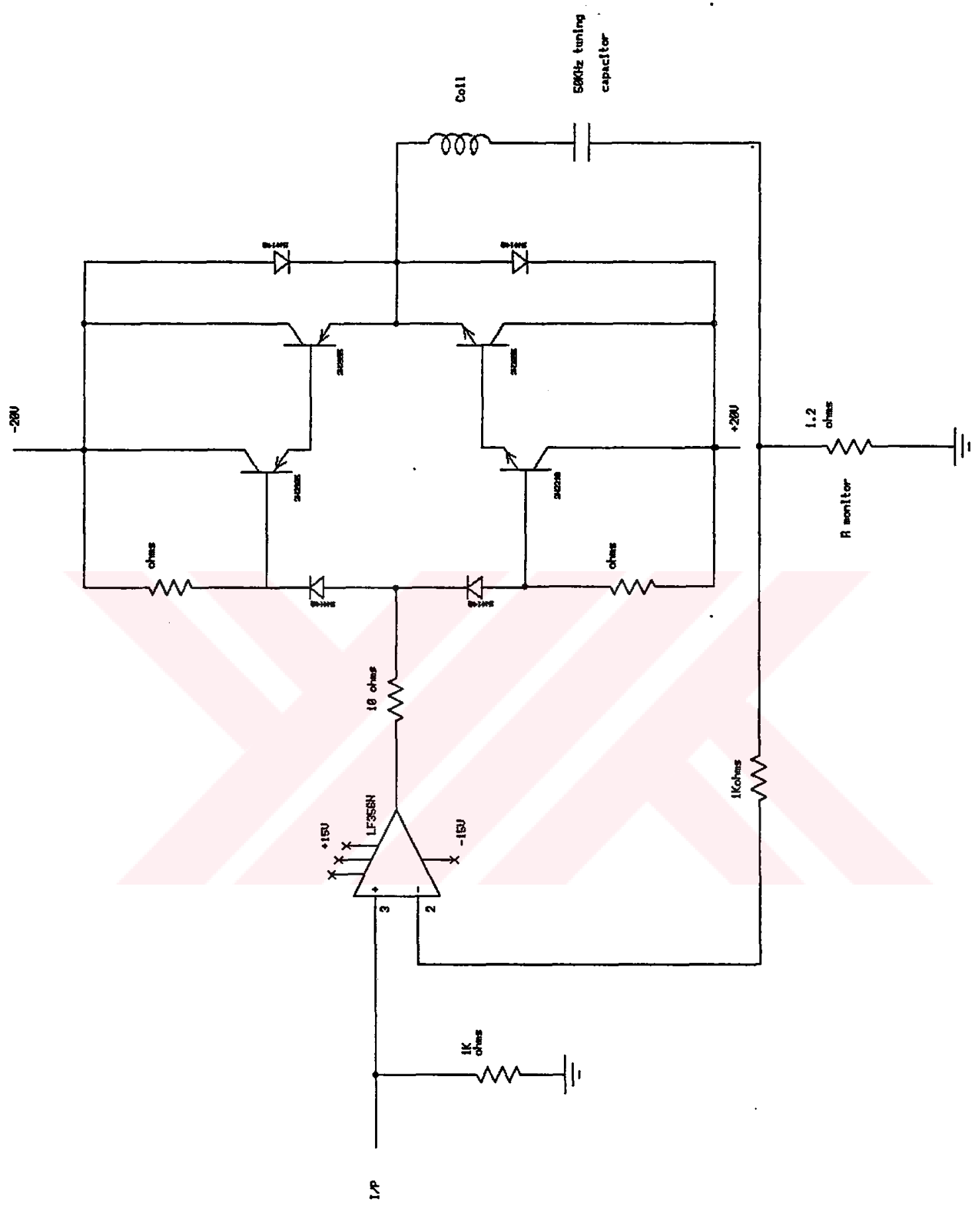

Figure 5.2. Circuit diagram of the power amplifier 
the two ends of the selected coil to the power amplifier. The coils are made up of copper wires which have a radius of $1 \mathrm{~mm}$. in order to provide self strength to preserve the circular coil profile. Each coil is a ten-turn coil of radius $24 \mathrm{~cm}$, which has an inductance of $L=565 \mu \mathrm{H}$ and resistance of $R=1.3 \Omega$. Although the current drive system is capable of selecting one of 16 coils, for simplicity only 6 off-centered coils are placed on the plane of the object region. At intersection points (at most 2 points), one of the coil wires is simply passed over another and again attached to the object plane. It is assumed that this kind of placement does not spoil the necessity of exciting the object with current carrying coils placed exactly on the object plane. 


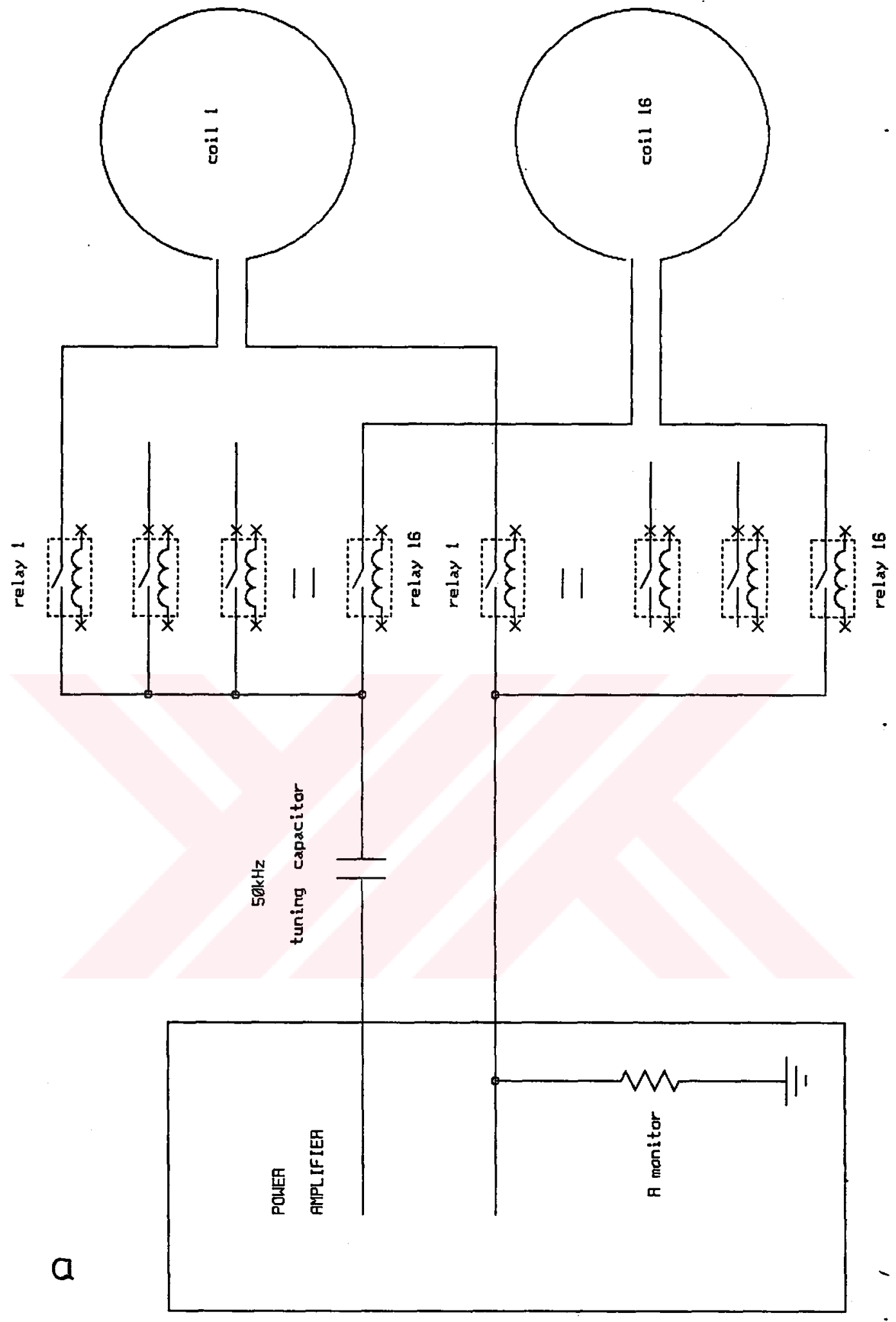

Figure 5.3. Circuit diagrams of (a) the overall current drive multiplexing circuit, (b) relay drive circuit 


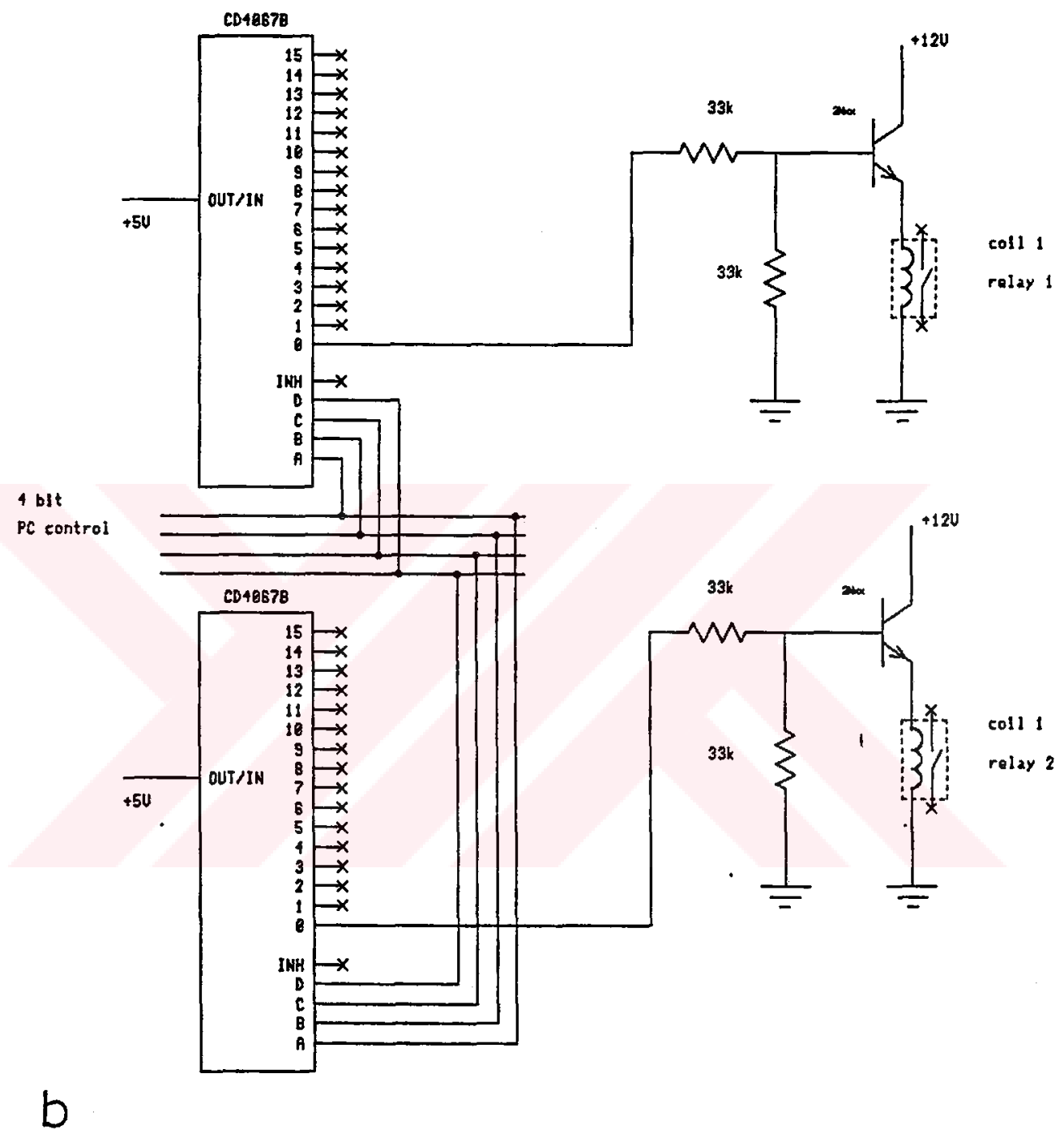

Figure 5.3 (cont'd) 


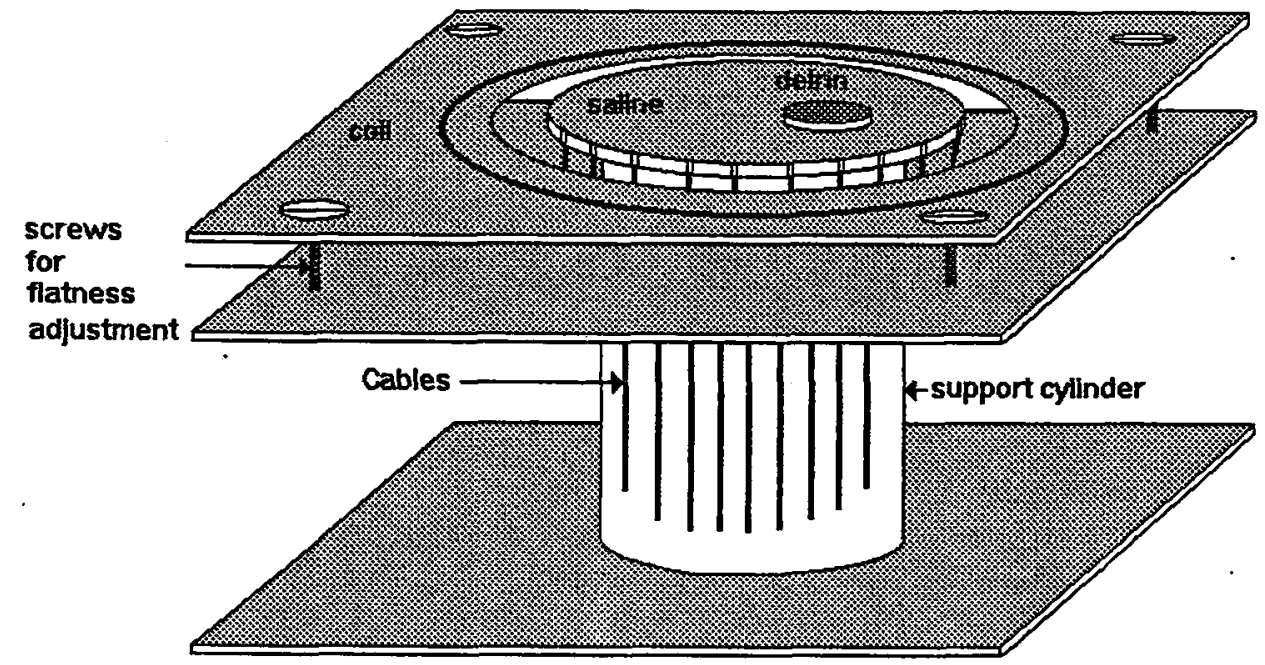

Figure 5.4. Measurement apparatus

The voltage measurements are performed by means of 16 brass electrodes mounted equidistantly around a circular dish of depth $1.5 \mathrm{~cm}$ made up of fiberglass. The circular dish is mounted on the top of a plastic pipe of height $50 \mathrm{~cm}$. which is fixed on the table by a planar wooden plate attached at its other end (Figure 5.4). The main goal of using such kind of apparatus is to make the measurement cables be fixed on the surface of the cylindrical pipe which makes them stand parallel to the created magnetic field direction and so minimize the induced electromotive force on these cables. The 16 voltage samples are brought to the buffers on the motherboard by screened cables which are attached to the electrodes by alligator clips. The screens are driven by the signal line on the buffer side (Figure 5.5). The output of 16 buffers are connected to the inputs of 2 analog multiplexer/demultiplexer (HEF 4067) whose select inputs are controlled by PC. The digital lines are isolated from the PC side by using the opto-isolation board given in Figure 5.6. 


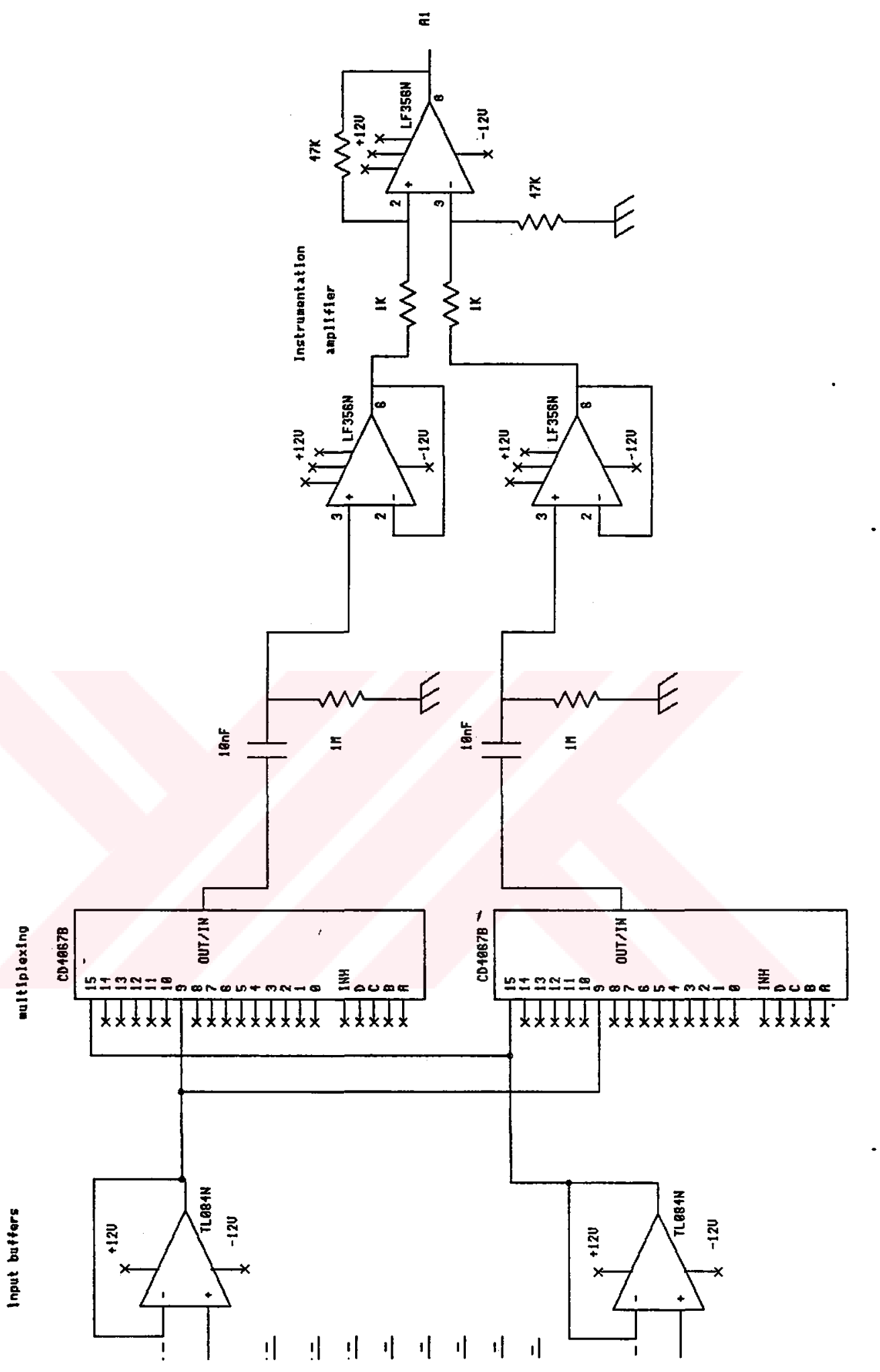

Figure 5.5. Circuit diagram of the voltage difference measurement system. 


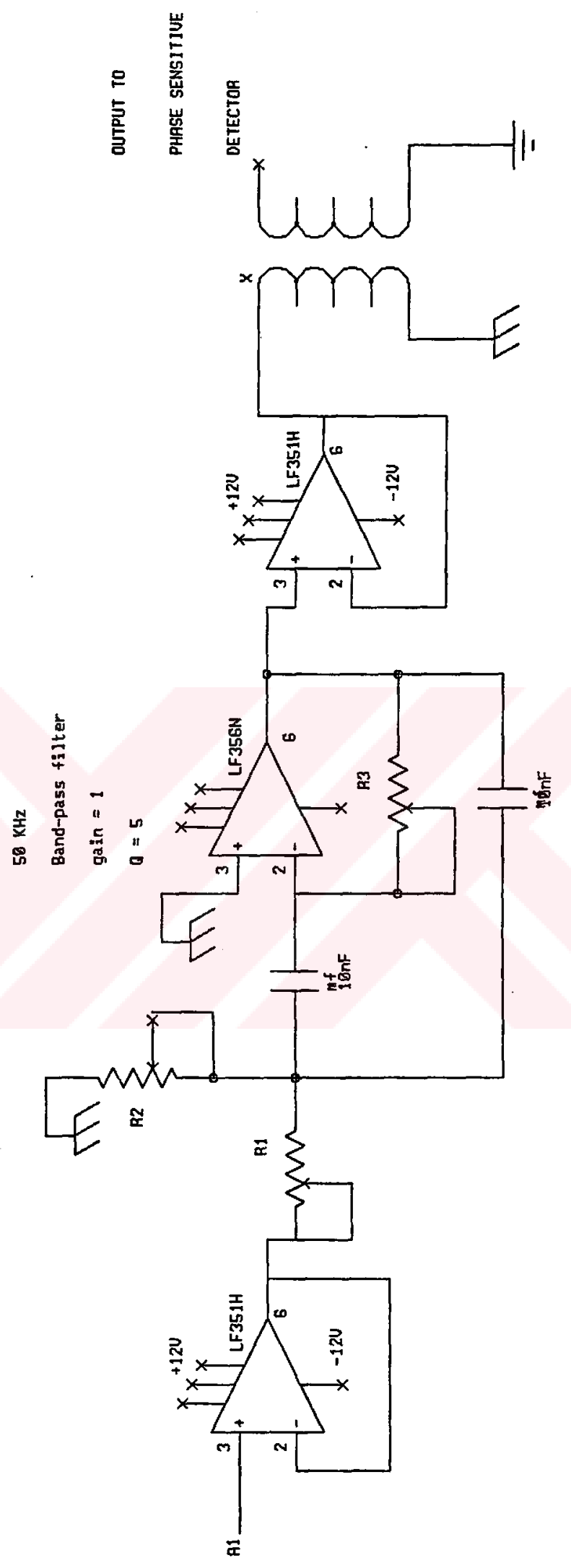

Figure 5.5 (cont'd) 


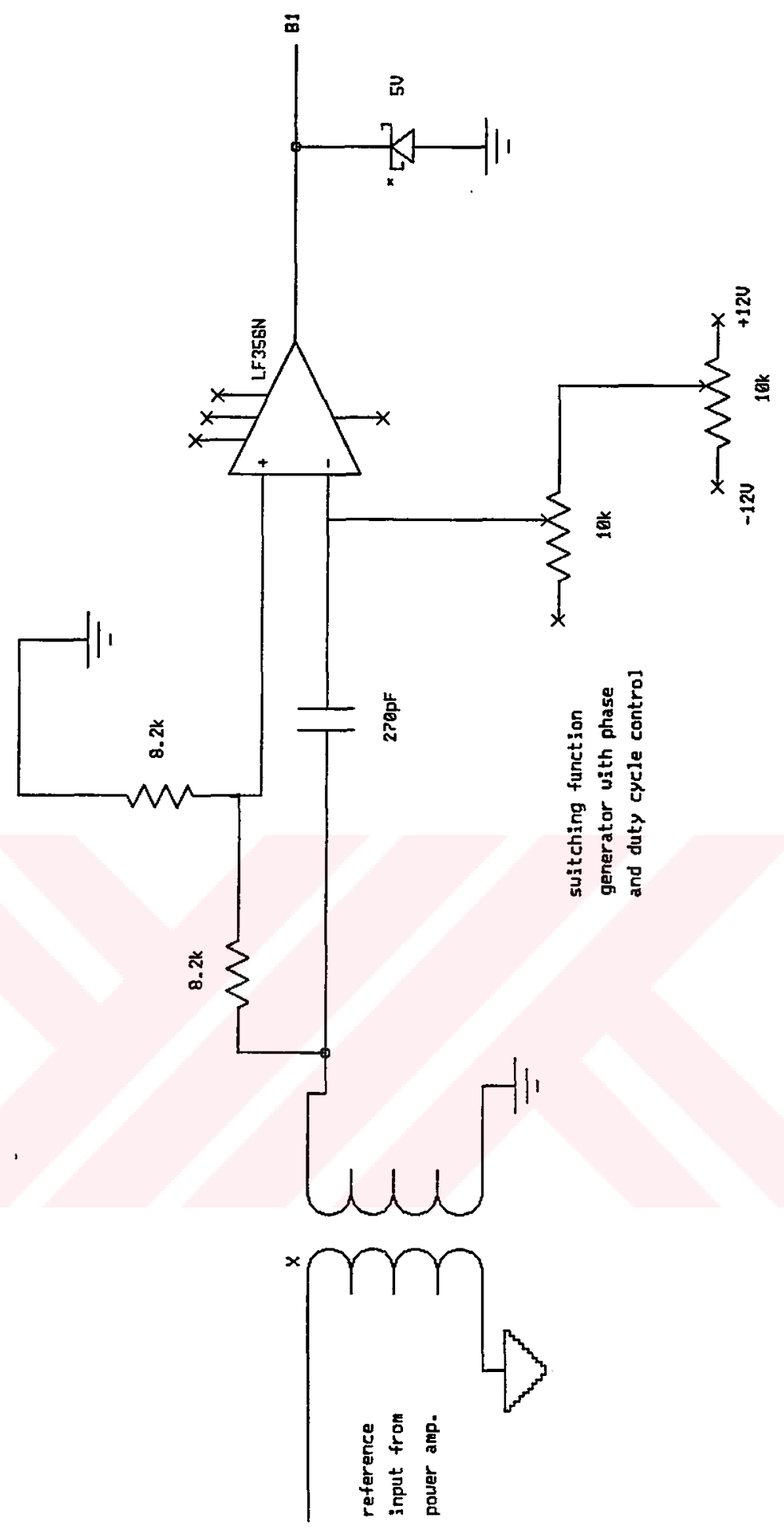

Figure 5.5 (cont'd) 


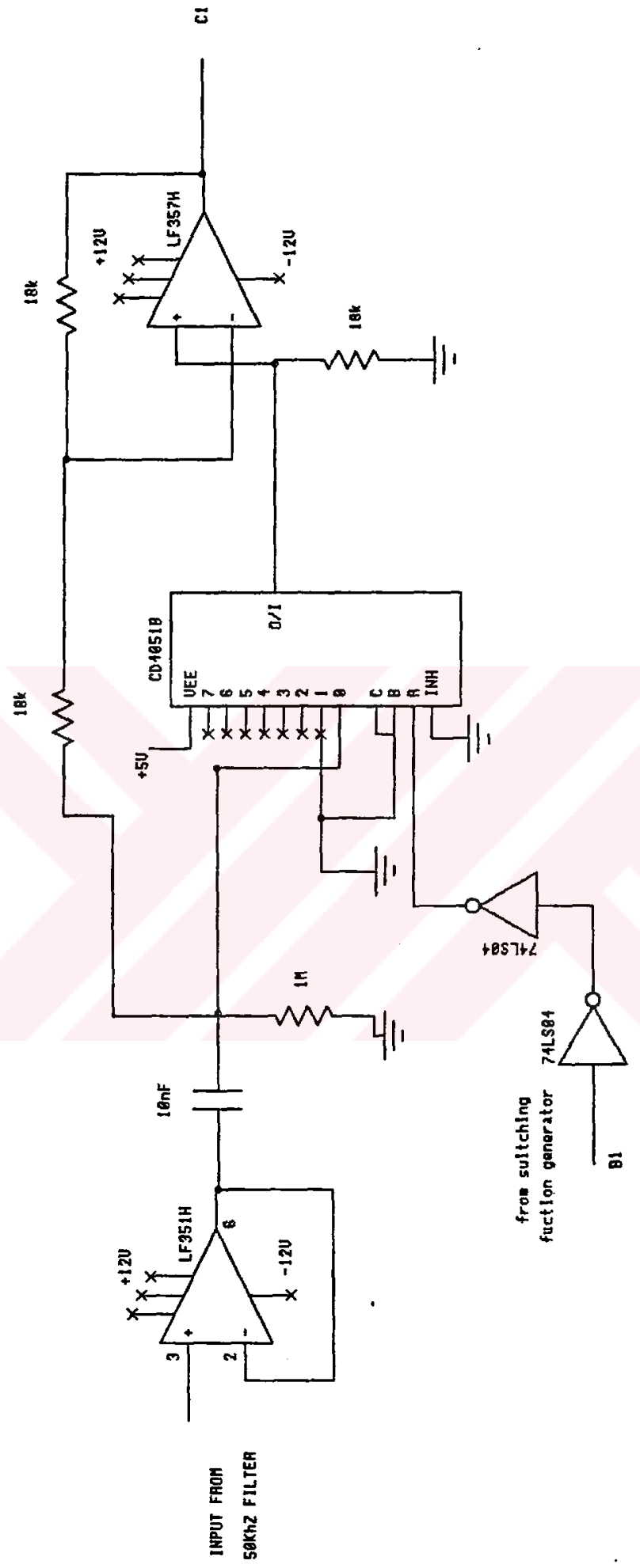

Figure 5.5 (cont'd) 


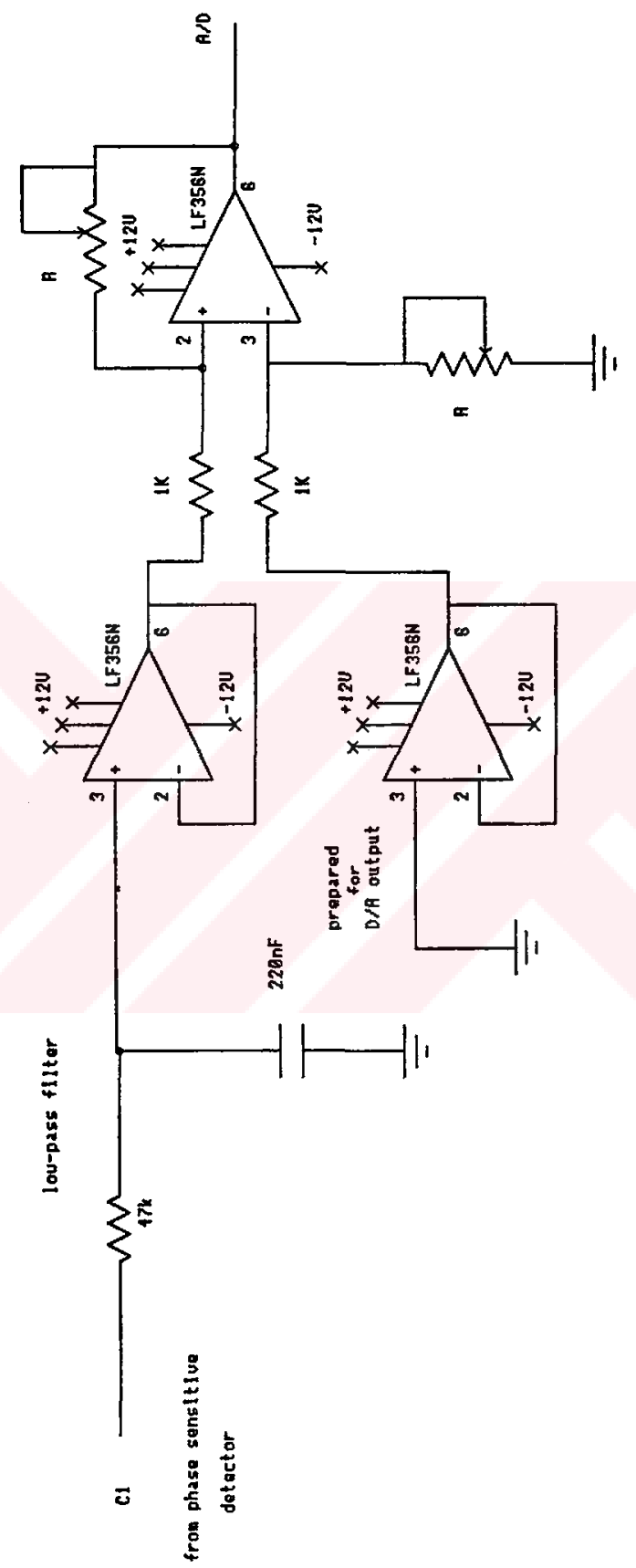

Figure 5.5 (cont'd) 


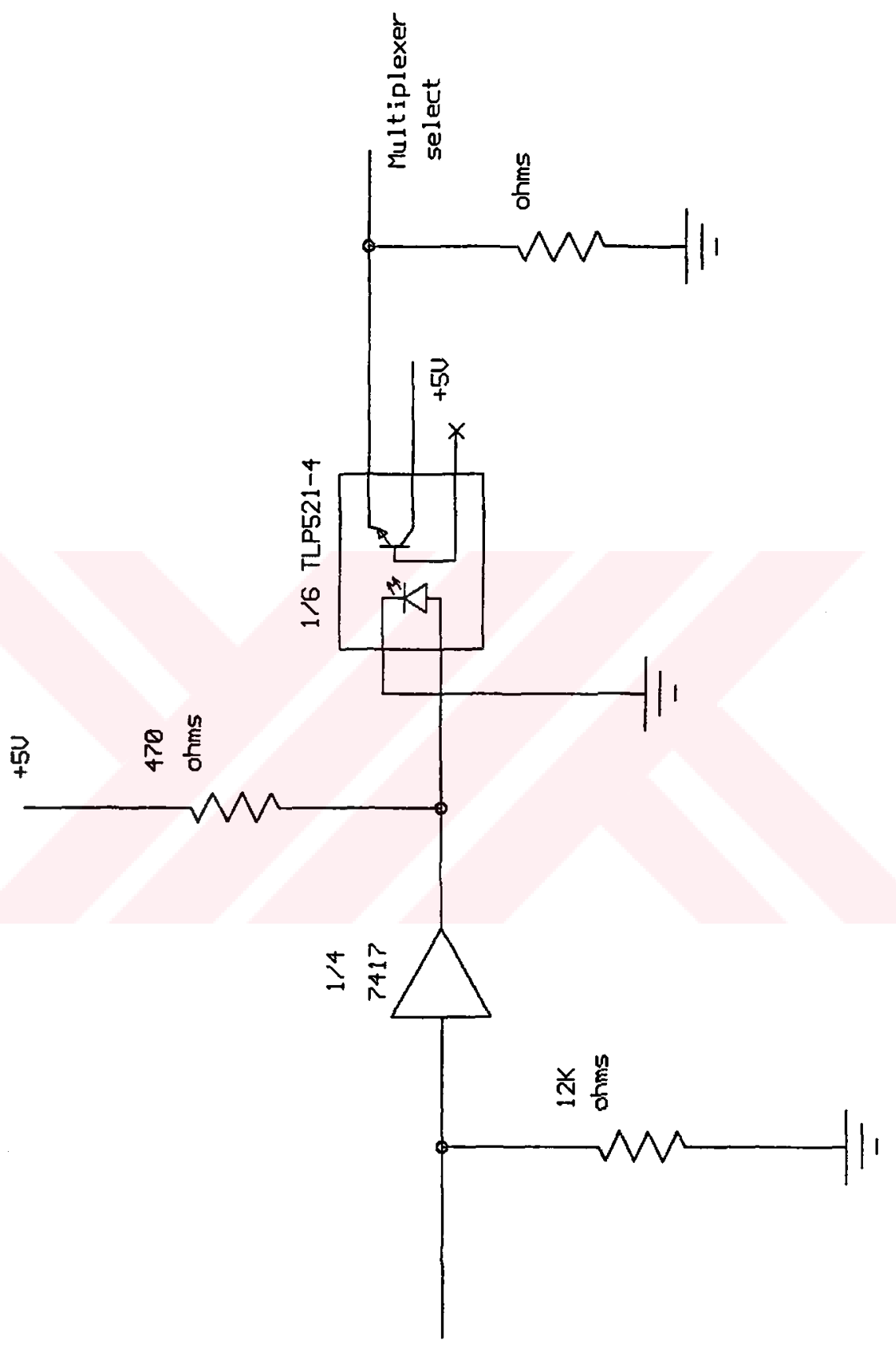

Figure 5.6. Circuit diagram of the opto-isolation circuit 
For each coil drive, 16 consecutive electrode pairs are selected providing 16 measurements. The difference of the voltages of the two selected electrodes is measured by an instrumentation amplifier which has a differential gain of $A_{d}=50$ and common mode gain of $A_{c}=0.01$ at $50 \mathrm{kHz}$. The output of the instrumentation amplifier is fed to an active filter which has a quality factor of $Q=4$ and gain $G=1$. The filter output is buffered and isolated from the PC side analog electronics using an isolation transformer. The quadrature component of the voltage measurement is obtained by means of a phase sensitive detector whose reference input is taken from the power amplifier monitor resistance $R_{m}$ by an isolation transformer and shifted by $90^{\circ}$. The output of the phase sensitive detector is low pass filtered by a simple R-C circuit with a time constant of 10 msec. This dc signal is amplified again by an amplifier which is initially designed to amplify the difference of two voltages, namely the instantaneous voltages measured for non-uniform object distribution and D/A output representing the corresponding data previously obtained from a uniform conductivity distribution. The aim of such a measurement strategy was to increase the number of bits in the digital representation of data which represents the existence of an inhomogeneity inside the object region. However, throughout this study the last stage differential amplifier is used as a simple inverting amplifier which has an adjustable feedback resistor to provide manual gain adjustment facility. Finally, the motherboard output is connected to a 12 bit A/D converter input of Labmaster Techmar and measured values are stored in a PC file. The difference of the data sets collected for uniform and non-uniform object distributions (i.e the potential difference data ) is obtained by software and used for image reconstruction.

\subsection{Timing}

A data collection routine is written which permits to change several parameters related with the timing of the data collection process. These parameters are : 1) number of $A / D$ conversions for each measurement, 2) delay between measurements, 3) number of repetitions of 16 measurements for a certain coil drive, 4) delay between coil drives. Depending on the value of these parameters the total data collection time can be calculated by the following 
formula :

$$
d c t=[n c \times a d c t+d m) \times n m \times n r+d c d] \times n c d
$$

where $d c d, n c, a d c t, d m, n m, n r, d c d, n c d$ denote the data conversion time, number of conversions, A/D conversion time, delay between measurements, number of measurements, number of repetitions, delay between coil drives and number of coil drives respectively.

The total time dct for a typical set of timing parameters ( $n c=$ $100, a d c t=50 \mu \mathrm{sec}, d m=100 \mathrm{msec}, n m=16, n r=10, d c d=1 \mathrm{sec}, \mathrm{ncd}=6) \mathrm{can}$ be calculated using this formula as

$$
d c t=[(100 \times 50 \mu s e c+100 \mathrm{msec}) \times 16 \times 10 \times 1 \mathrm{sec}] \times 6=89 \mathrm{sec} .
$$

\subsection{Experimental Results}

\subsubsection{Data Comparison}

Two experiments are designed to check the consistency of the theoritical results with real data. For each experiment, the uniform object distribution is prepared by filling saline solution inside the constructed dish which has a $1 \% \mathrm{gr} \mathrm{NaCl}$ in weight. The inhomogeneity is represented by a circular disk of delrin which has a radius of $3 \mathrm{~cm}$.

In the first experiment, a circular coil of radius $23.5 \mathrm{~cm}$ is placed around the object region by taking care of the coincidence of the centers of the coil and circular dish at the same point. After collecting data for saline solution (data for uniform conductivity distribution) the delrin disk is inserted in the saline solution centring the object region. The resultant object distribution is used for collecting data for non-uniform conductivity distribution. For the first set of data, since the normal component of magnetic vector potential is zero along the boundary, the potential differences will be zero. The nonzero component of the voltage gradients will be the induced electromotive force (i.e caused 
by the $-\omega \vec{A}$ term of the electric field). For the non-uniform object distribution, since the normal component of the magnetic vector potential will be zero both along the boundaries of the delrin disk and object region, again no potential differences should appear. The nonzero component of the measured voltages is again caused by $-\omega \vec{A}$ term of the electric field. Since $-\omega \vec{A}$ is expected to be same for both uniform and non-uniform object distributions (as claimed in section 2.3), the placement of the delrin disk at the center of the object region should not affect the boundary voltage measurements. This expectation is verified experimentally, i.e no significant voltage change is observed.

In the second experiment, the data for non-uniform conductivity distribution is obtained for the coil configuration and object distribution shown in Figure 5.7(a). The potential gradients are compared with the results obtained by FEM solutions. This comparison will also take into account the errors caused by inadequate representation of the actual conductivity distribution by using triangular elements. The scaled real data and FEM solutions are given in Table 5.1. It is observed that the realized data acqusition system is capable of collecting real data which closely follows the expected boundary potential differences. Major differences appear when the sign of the gradients changes along the object boundary. Several factors that influence this comparison are : 1) inadequate representation of conductivity distribution (i.e triangular elements are used), 2) inaccurate placement of delrin (placed manually), 3) misplaced electrodes, 4) misplaced coil and imperfect coil shape (i.e deviations from circular shape and finite thickness of wires), 5) inadequate flatness adjustment mechanism for the saline filled phantom, 6) electrical noise on the realized hardware. The results of the two experiment show that the realized hardware performs sufficiently good measurements to support the formulations derived for the behaviour of scalar potential distrtibution in conductive objects when excited by time varying fields at low frequencies. The consistency of the theoretical studies with the experimental results can also be tested by using collected data to reconstruct the conductivity images of real objects. In the following section, studies about image reconstruction based on the formulations given in Chapter 4 will be presented using real data. 
Table 5.1. Comparison of the calculated and measured boundary potential differences. Computer simulation results are obtained by FEM. $\Delta \bar{g}$ represents the difference of boundary potential gradients obtained for uniform and non-uniform conductivity distributions

\begin{tabular}{||c|c|c||}
\hline$\Delta \bar{g}$ & $\begin{array}{c}\text { calculated } \\
\text { potential } \\
\text { differences }(m V)\end{array}$ & $\begin{array}{c}\text { measured } \\
\text { potential } \\
\text { differences }(m V)\end{array}$ \\
\hline 1 & 0.2768 & 0.3172 \\
2 & 0.2303 & 0.2041 \\
3 & 0.1703 & 0.1729 \\
4 & 0.1326 & 0.1392 \\
5 & 0.1119 & 0.1229 \\
6 & 0.1004 & 0.1087 \\
7 & 0.0973 & 0.0875 \\
8 & 0.1005 & 0.1163 \\
9 & 0.1107 & 0.1298 \\
10 & 0.1310 & 0.1311 \\
11 & 0.1668 & 0.1722 \\
12 & 0.2239 & 0.2397 \\
13 & 0.2557 & 0.2222 \\
14 & -0.2627 & -0.5459 \\
15 & -1.6187 & -1.6187 \\
16 & -0.2274 & -0.0410 \\
\hline
\end{tabular}




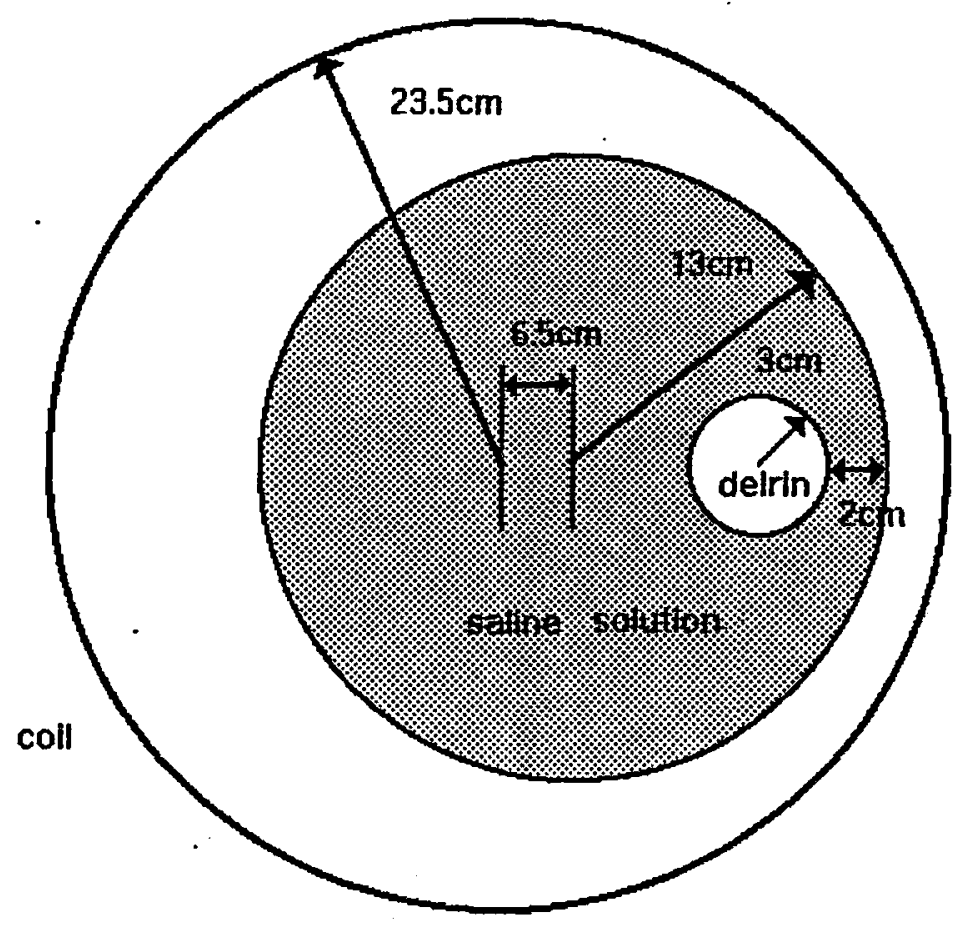

Figure 5.7. Coil configuration and object distribution for the second experiment

\subsubsection{Image Reconstruction Using Real Data}

It is shown in section 4.3 that, in the case of noiseless data (i.e without measurement noise and ineffective numerical noise) it is possible to use all the terms in the series given by equation (4.6) for the solution of $\Delta \bar{\sigma}$. However, in the case of real data, the series has to be truncated at a summation index $k$ depending on the signal to noise ratio (SNR) of the data acquisition system. In this study, this optimum point of truncation is found by repeatedly solving the inverse problem with different number of terms. The number of terms included in the series is determined by assuming different values for SNR of the realized data acqusition system.

Four set of data is collected by manually placing the delrin disk at different locations in the saline solution along a radial line. The resultant object distributions are given in Figure 5.8. In order to increase the number of independent measurements and so to reconstruct better images six coils are located on the object plane as shown in Figure 5.9. This coil configuration is 

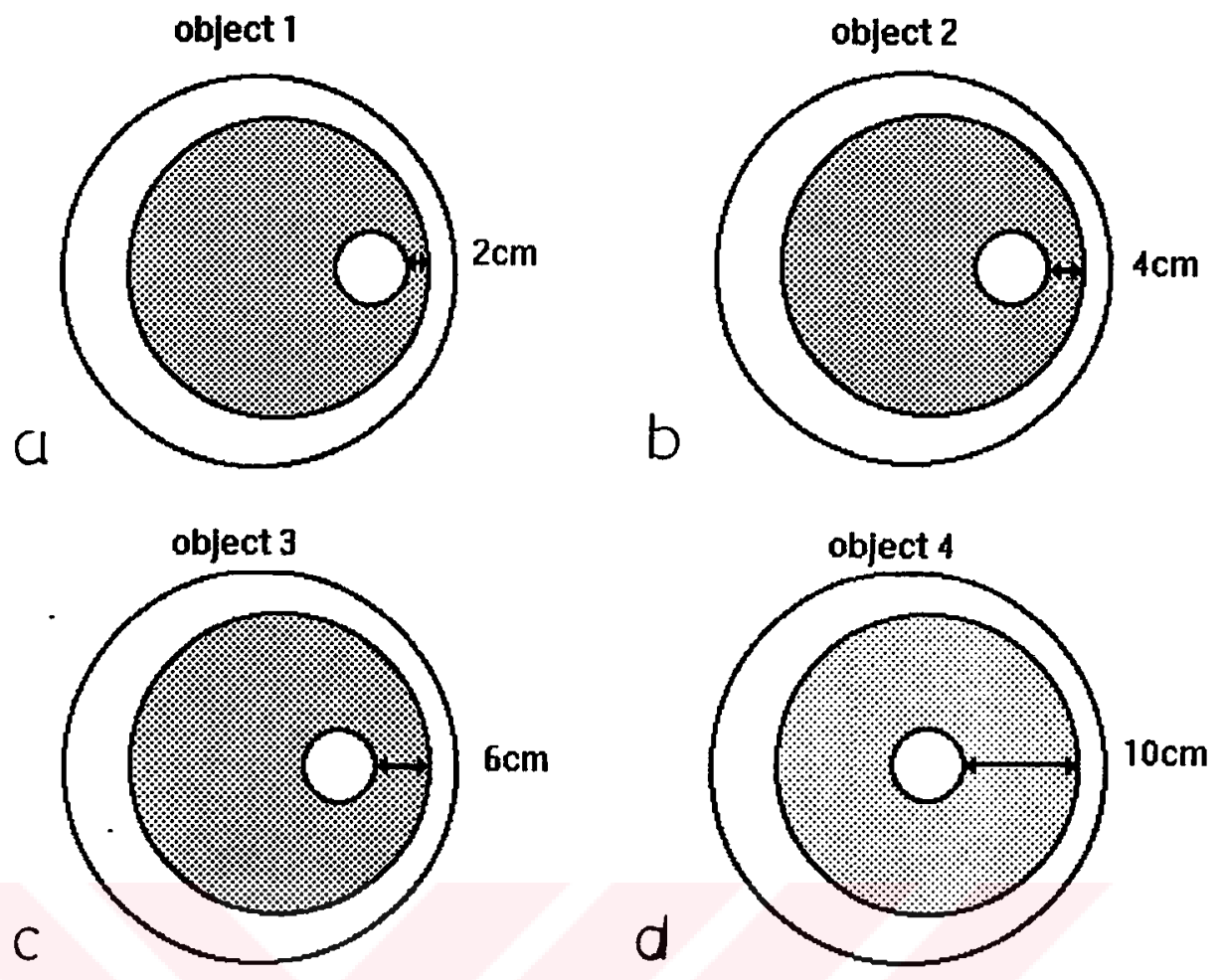

Figure 5.8. Four different object distributions obtained by placing the delrin disk at different locations inside the saline solution

particularly selected in order to place the coils easily. For each coil placement any two opposite edges of the coil are first marked. Then the coil is placed in such a way that the two marks on the coil and the two opposing electrodes are located on the same line. For example, the first coil is placed such that the marked edges ( $A$ and $B$ ) of the coil are on the same line with the two electrodes numbered as 0 and 8 . The distance $0 B$ is adjusted manually depending on the amount of coil center shift and measured simply by using a ruler. In this study, the coils are shifted by $6.5 \mathrm{~cm}$ with respect to the center of the object region. Since there are 16 electrodes along the object boundary 16 voltage differences are measured for each coil configuration yielding 96 measurements (90 of which are independent). The potential differences obtained for the four object distributions are given in Tables 5.2, 5.3, 5.4 and 5.5, respectively. For each object distribution 6 different images are reconstructed. In each case SNR is assumed to take six different values. These SNR values, the corresponding truncation level singular values and their number of terms used in image reconstruction 


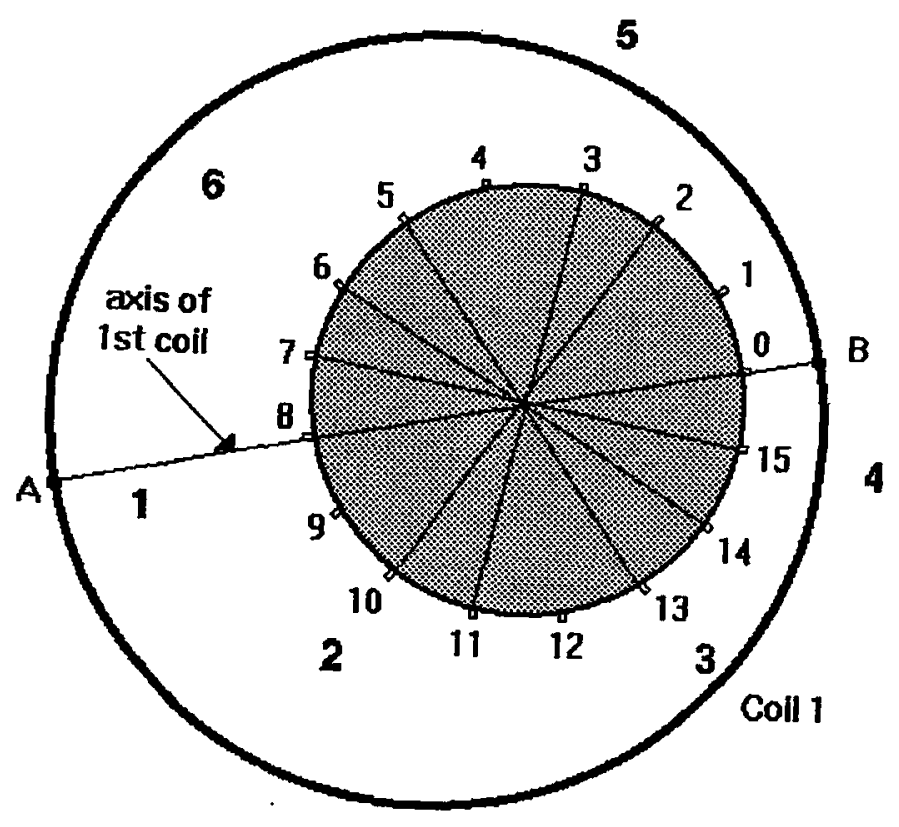

Figure 5.9. Coil placement strategy. 6 circular coils of radii $24.5 \mathrm{~cm}$ are placed in such a way that centers are shifted by $6.5 \mathrm{~cm}$ from the object origin. Only the placement of the first coil is shown. The two marks on the coil and the two opposing electrodes ( $0^{\text {th }}$ and $8^{\text {th }}$ electrode) are located on the same line.

are given in Table 5.6. The reconstructed images are given in Figure 5.10, 5.11, 5.12 and 5.13, respectively. It can easily be observed that, the measured boundary potential gradients are accurate enough to provide good estimates of actual object distribution. The best images, in each case, are obtained when singular values smalled than $0.00125 \lambda_{1}$ are truncated which shows that the effective SNR of this system is approximately $800(58 \mathrm{~dB})$. 
Table 5.2. Collected data for the first object distribution (see Figure 5.8 (a))

\begin{tabular}{||c|c|c|c|c|c|c||}
\hline$\Delta \bar{g}$ & $\begin{array}{c}(V) \\
\text { coil 1 }\end{array}$ & $\begin{array}{c}(V) \\
\text { coil 2 }\end{array}$ & $\begin{array}{c}(V) \\
\text { coil 3 }\end{array}$ & $\begin{array}{c}(V) \\
\text { coil 4 }\end{array}$ & $\begin{array}{c}(V) \\
\text { coil 5 }\end{array}$ & $\begin{array}{c}(V) \\
\text { coil 6 }\end{array}$ \\
\hline 1 & 0.1486 & 0.1575 & 0.1568 & 0.1415 & 0.1250 & 0.1348 \\
2 & 0.0956 & 0.1012 & 0.0970 & 0.0928 & 0.0870 & 0.0915 \\
3 & 0.0810 & 0.0773 & 0.0797 & 0.0754 & 0.0700 & 0.0767 \\
4 & 0.0652 & 0.0659 & 0.0615 & 0.0627 & 0.0628 & 0.0650 \\
5 & 0.0576 & 0.0550 & 0.0562 & 0.0548 & 0.0523 & 0.0554 \\
6 & 0.0509 & 0.0464 & 0.0465 & 0.0467 & 0.0442 & 0.0480 \\
7 & 0.0410 & 0.0487 & 0.0429 & 0.0550 & 0.0555 & 0.0441 \\
8 & 0.0545 & 0.0500 & 0.0516 & 0.0486 & 0.0500 & 0.0512 \\
9 & 0.0608 & 0.0568 & 0.0592 & 0.0577 & 0.0600 & 0.0590 \\
10 & 0.0614 & 0.0601 & 0.0615 & 0.0617 & 0.0665 & 0.0619 \\
11 & 0.0807 & 0.0765 & 0.0805 & 0.0788 & 0.0865 & 0.0830 \\
12 & 0.1123 & 0.1085 & 0.1094 & 0.1183 & 0.1287 & 0.1190 \\
13 & 0.1041 & 0.0951 & 0.0952 & 0.1035 & 0.1335 & 0.1212 \\
14 & -0.2557 & -0.2738 & -0.2673 & -0.2469 & -0.214 & -0.2200 \\
15 & -0.7582 & -0.7513 & -0.7365 & -0.7300 & -0.7641 & -0.7553 \\
16 & -0.0192 & 0.0023 & -0.0071 & -0.0266 & -0.0564 & -0.0424 \\
\hline
\end{tabular}


Table 5.3. Collected data for the second object distribution (see Figure 5.8 (b)).

\begin{tabular}{||c|c|c|c|c|c|c||}
\hline$\Delta \bar{g}$ & $\begin{array}{c}(V) \\
\text { coil 1 }\end{array}$ & $\begin{array}{c}(V) \\
\text { coil 2 }\end{array}$ & $\begin{array}{c}(V) \\
\text { coil 3 }\end{array}$ & $\begin{array}{c}(V) \\
\text { coil 4 }\end{array}$ & $\begin{array}{c}(V) \\
\text { coil } 5\end{array}$ & $\begin{array}{c}(V) \\
\text { coil } 6\end{array}$ \\
\hline 1 & 0.0910 & 0.1438 & 0.1359 & 0.0927 & 0.0168 & 0.0372 \\
2 & 0.1338 & 0.1672 & 0.1755 & 0.1534 & 0.1034 & 0.1070 \\
3 & 0.1372 & 0.1629 & 0.1820 & 0.1644 & 0.1241 & 0.1183 \\
4 & 0.1383 & 0.1561 & 0.1704 & 0.1656 & 0.1387 & 0.1277 \\
5 & 0.1145 & 0.1305 & 0.1490 & 0.1483 & 0.1244 & 0.1116 \\
6 & 0.1004 & 0.1093 & 0.1233 & 0.1298 & 0.1119 & 0.0970 \\
7 & 0.1158 & 0.1256 & 0.1423 & 0.0000 & 0.1419 & 0.1193 \\
8 & 0.1068 & 0.1160 & 0.1304 & 0.1361 & 0.1287 & 0.1099 \\
9 & 0.1203 & 0.1231 & 0.1408 & 0.1509 & 0.1516 & 0.1283 \\
10 & 0.1131 & 0.1122 & 0.1284 & 0.1419 & 0.1529 & 0.1244 \\
11 & 0.1358 & 0.1253 & 0.1403 & 0.1563 & 0.1807 & 0.1495 \\
12 & 0.1565 & 0.1289 & 0.1374 & 0.1626 & 0.2187 & 0.1844 \\
13 & -0.0365 & -0.0837 & -0.0835 & -0.0431 & 0.0466 & 0.0328 \\
14 & -0.4488 & -0.5184 & -0.5521 & -0.5209 & -0.4178 & -0.3816 \\
15 & -0.7424 & -0.7869 & -0.8641 & -0.8814 & -0.8564 & -0.7595 \\
16 & -0.2380 & 0.2143 & -0.2600 & -0.3101 & -0.3706 & -0.3065 \\
\hline
\end{tabular}


Table 5.4. Collected data of the third object distribution (see Figure 5.8 (a)).

\begin{tabular}{||c|c|c|c|c|c|c||}
\hline$\Delta \bar{g}$ & $\begin{array}{c}(V) \\
\text { coil 1 }\end{array}$ & $\begin{array}{c}(V) \\
\text { coil 2 }\end{array}$ & $\begin{array}{c}(V) \\
\text { coil 3 }\end{array}$ & $\begin{array}{c}(V) \\
\text { coil 4 }\end{array}$ & $\begin{array}{c}(V) \\
\text { coil 5 }\end{array}$ & $\begin{array}{c}(V) \\
\text { coil 6 }\end{array}$ \\
\hline 1 & -0.0241 & 0.0169 & -0.0035 & -0.0469 & -0.1073 & -0.0727 \\
2 & 0.0266 & 0.0596 & 0.0667 & 0.0427 & -0.0145 & 0.0003 \\
3 & 0.0520 & 0.0862 & 0.1091 & 0.0921 & 0.0378 & 0.0342 \\
4 & 0.0719 & 0.0992 & 0.1257 & 0.1194 & 0.0748 & 0.0598 \\
5 & 0.0669 & 0.0885 & 0.1158 & 0.1158 & 0.0828 & 0.0614 \\
6 & 0.0579 & 0.0768 & 0.1016 & 0.1085 & 0.0847 & 0.0599 \\
7 & 0.0678 & 0.0803 & 0.1071 & 0.0000 & 0.0965 & 0.0702 \\
8 & 0.0549 & 0.0663 & 0.0931 & 0.1036 & 0.0921 & 0.0628 \\
9 & 0.0583 & 0.0627 & 0.0881 & 0.1051 & 0.1038 & 0.0713 \\
10 & 0.0460 & 0.0418 & 0.0616 & 0.0821 & 0.0914 & 0.0628 \\
11 & 0.0404 & 0.0279 & 0.0415 & 0.0673 & 0.0948 & 0.0638 \\
12 & 0.0234 & -0.0076 & -0.0041 & 0.0249 & 0.0823 & 0.0579 \\
13 & -0.0447 & -0.0990 & -0.1213 & -0.0942 & -0.0019 & -0.0007 \\
14 & -0.1413 & -0.2008 & -0.2536 & -0.2450 & -0.1572 & -0.1154 \\
15 & -0.2286 & -0.2719 & -0.3467 & -0.3690 & -0.3275 & -0.2405 \\
16 & -0.1260 & 0.1261 & -0.1792 & -0.2204 & -0.2386 & -0.1708 \\
\hline
\end{tabular}


Table 5.5. Collected data for the fourth object distribution (see Figure 5.8 (a)).

\begin{tabular}{||c|c|c|c|c|c|c||}
\hline$\Delta \bar{g}$ & $\begin{array}{c}(V) \\
\text { coil 1 }\end{array}$ & $\begin{array}{c}(V) \\
\text { coil 2 }\end{array}$ & $\begin{array}{c}(V) \\
\text { coil 3 }\end{array}$ & $\begin{array}{c}(V) \\
\text { coil 4 }\end{array}$ & $\begin{array}{c}(V) \\
\text { coil } 5\end{array}$ & $\begin{array}{c}(V) \\
\text { coil } 6\end{array}$ \\
\hline 1 & 0.0677 & 0.0812 & 0.0487 & 0.01121 & -0.0063 & 0.0448 \\
2 & 0.0216 & 0.0383 & 0.0275 & -0.0039 & -0.0406 & -0.0084 \\
3 & 0.0061 & 0.0333 & 0.0383 & 0.0093 & -0.0456 & -0.0240 \\
4 & -0.0136 & 0.0221 & 0.0444 & 0.0254 & -0.0452 & -0.0391 \\
5 & -0.0394 & -0.0066 & 0.0303 & 0.0253 & -0.0423 & -0.0549 \\
6 & -0.0623 & -0.0351 & 0.0069 & 0.0183 & -0.0380 & -0.0658 \\
7 & -0.0615 & -0.0432 & 0.0053 & 0.0000 & -0.0107 & -0.0558 \\
8 & -0.0651 & -0.0608 & -0.0219 & 0.0099 & -0.0035 & -0.0532 \\
9 & -0.0388 & -0.0521 & -0.0271 & 0.0093 & 0.0257 & -0.0255 \\
10 & -0.0360 & -0.0558 & -0.0457 & -0.0162 & 0.0235 & -0.0128 \\
11 & -0.0168 & -0.0414 & -0.0471 & -0.0225 & 0.0353 & 0.0142 \\
12 & 0.0190 & -0.0139 & -0.0366 & -0.0199 & 0.0495 & 0.0459 \\
13 & 0.0516 & 0.0164 & -0.0207 & -0.0174 & 0.0561 & 0.0725 \\
14 & 0.0434 & 0.0185 & -0.0199 & -0.0253 & 0.0271 & 0.0576 \\
15 & 0.0539 & 0.0383 & -0.0787 & -0.0291 & 0.0070 & 0.0536 \\
16 & 0.0632 & 0.0598 & -0.0220 & -0.0040 & 0.00515 & 0.0518 \\
\hline
\end{tabular}



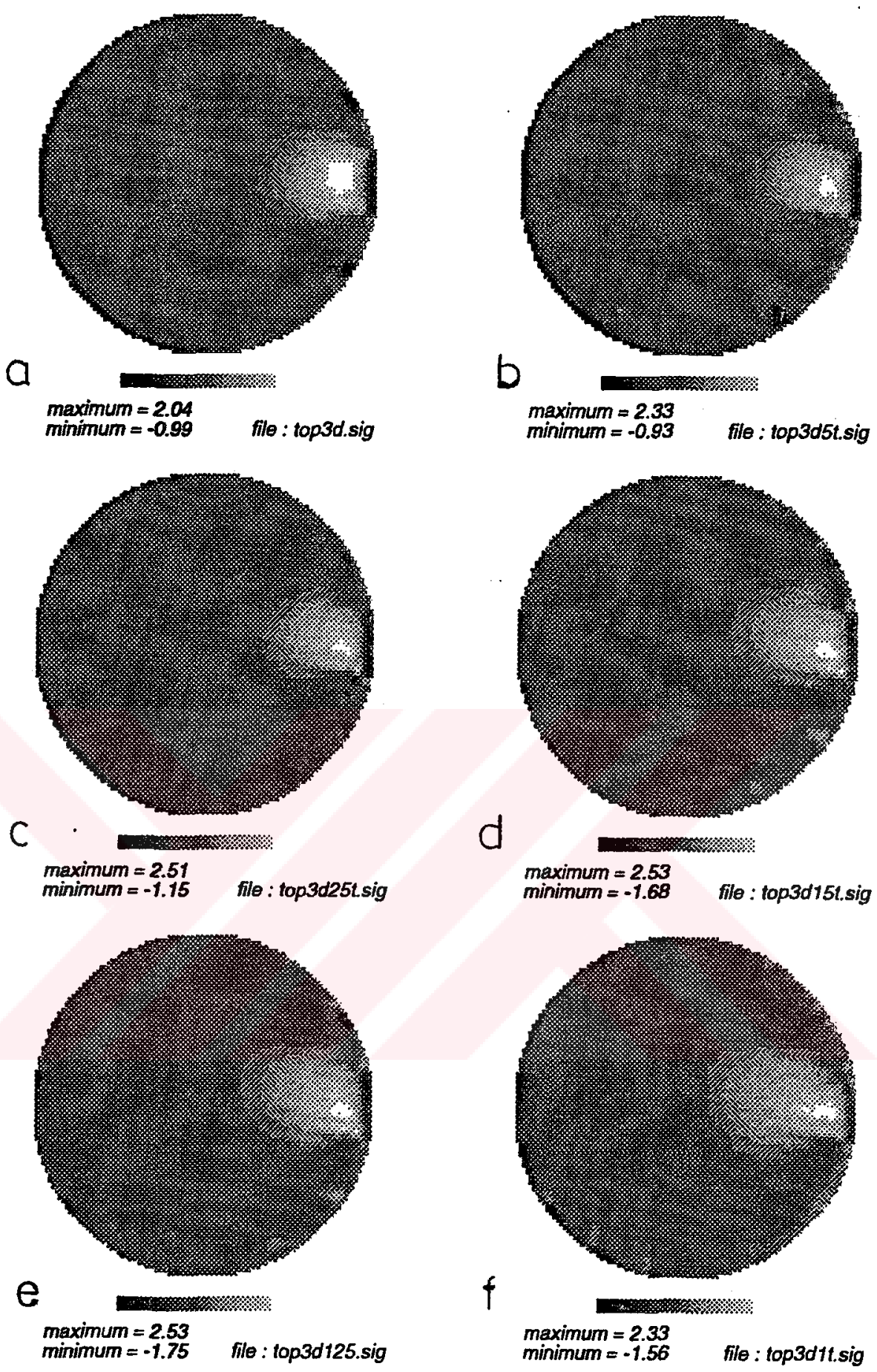

Figure 5.10. Recontructed images of the first object distribution assuming different values for $S N R$ (a) $S N R=100$, (b) $S N R=200$, (c) $S N R=400$, (d) $S N R=657$, (e) $\mathrm{SNR}=800$, (f) $\mathrm{SNR}=1000$ 

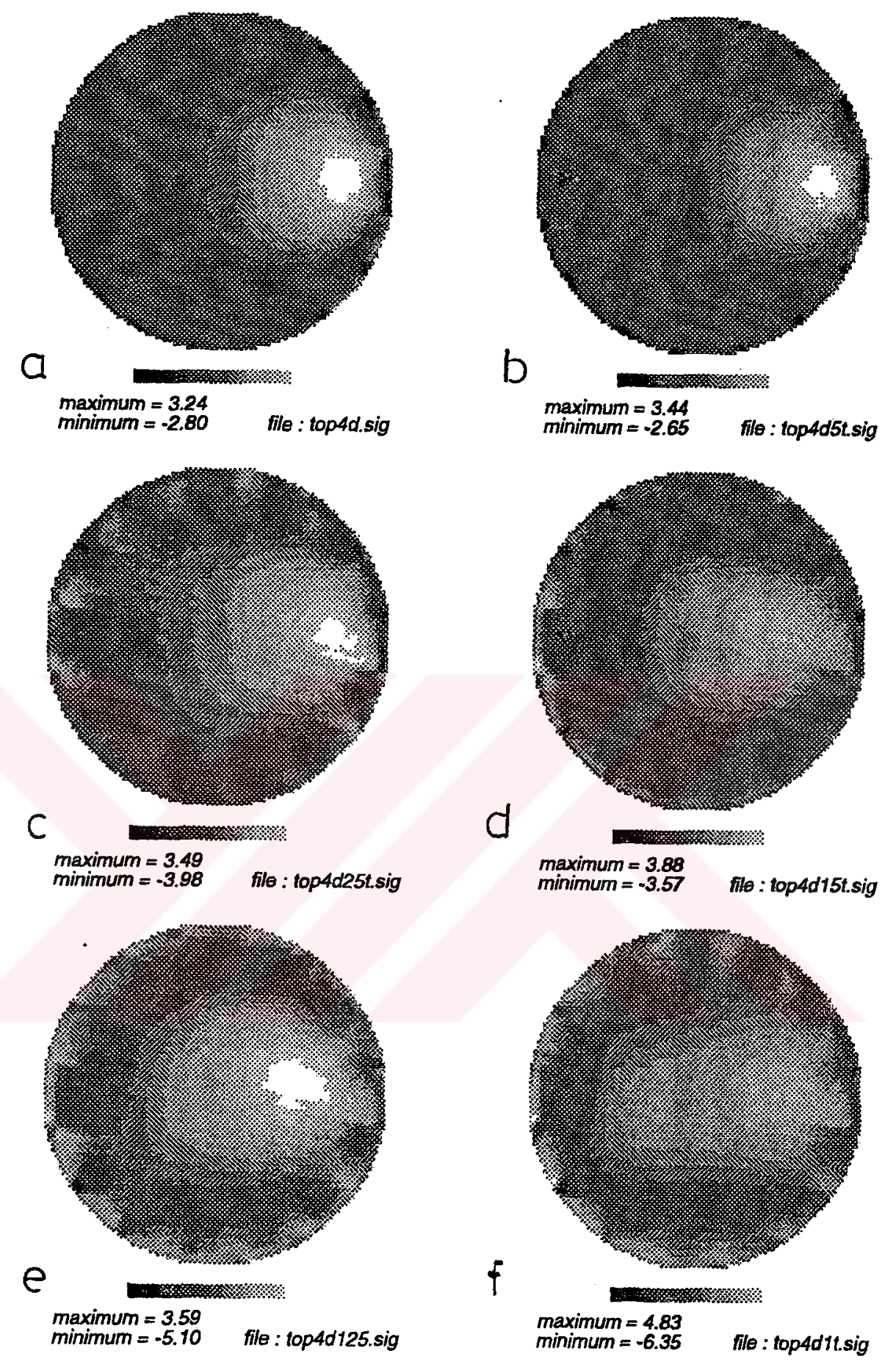

Figure 5.11. Recontructed images of the second object distribution assuming different values for SNR (a) SNR=100, (b) $S N R=200$, (c) $S N R=400$, (d) $\mathrm{SNR}=657$, (e) $\mathrm{SNR}=800$, (f) $\mathrm{SNR}=1000$ 

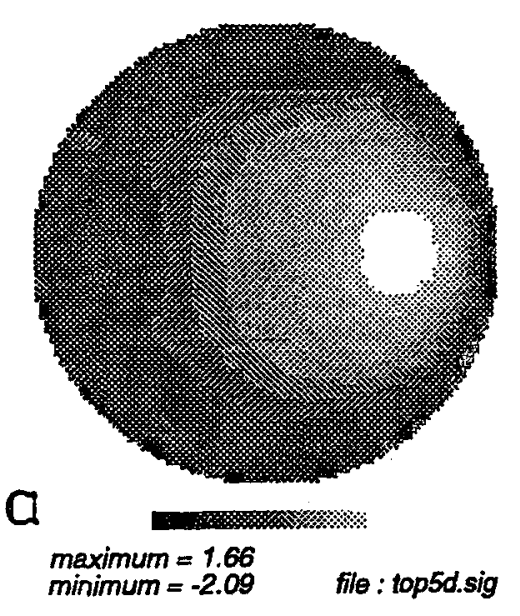

b
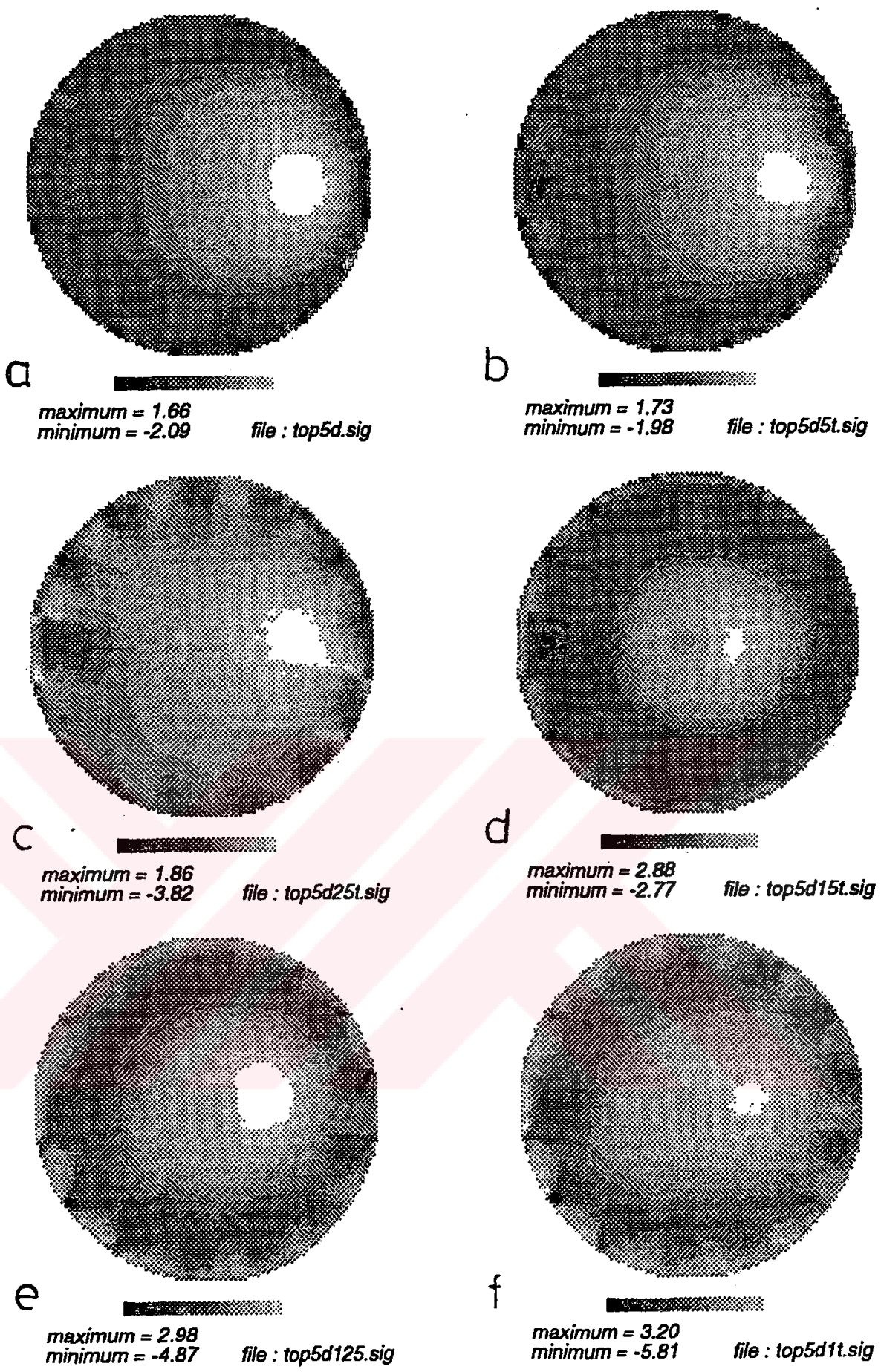

Figure 5.12. Recontructed images of the third object distribution assuming different values for SNR (a) SNR=100, (b) $S N R=200$, (c) $S N R=400$, (d) $\mathrm{SNR}=657$, (e) $\mathrm{SNR}=800$, (f) $\mathrm{SNR}=1000$ 

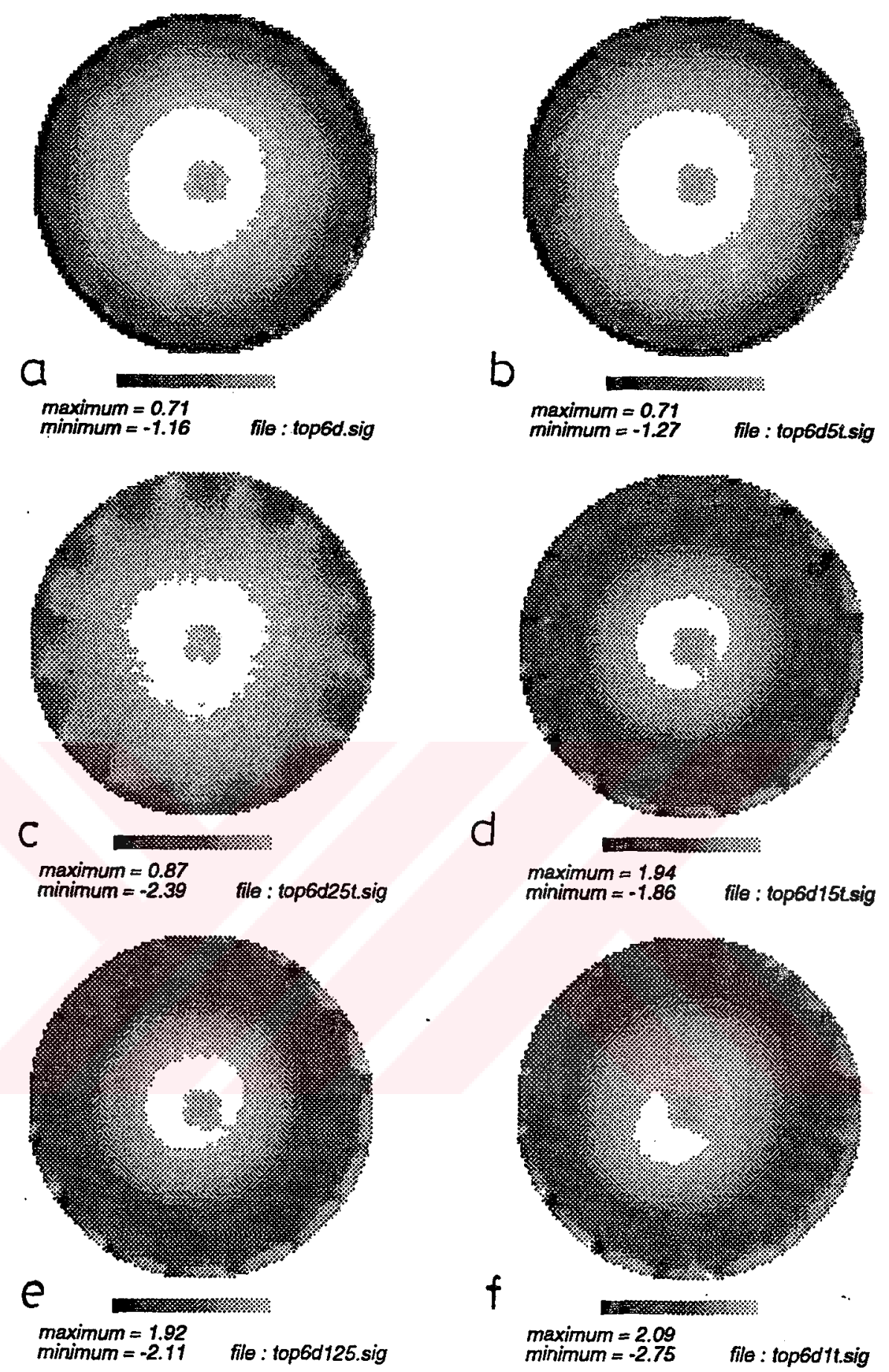

Figure 5.13. Recontructed images of the fourth object distribution assuming different values for $\mathrm{SNR}$ (a) $\mathrm{SNR}=100$, (b) $\mathrm{SNR}=200$, (c) $\mathrm{SNR}=400$, (d) $\mathrm{SNR}=657$, (e) $\mathrm{SNR}=800$, (f) $\mathrm{SNR}=1000$ 
Table 5.6. Truncation level singular values and number of terms included in image reconstruction due to assigned six different SNR

\begin{tabular}{||c|c|c||}
\hline SNR & $\lambda_{k}$ & no. of terms \\
\hline 100 & $0.01000 \lambda_{1}$ & 31 \\
200 & $0.00500 \lambda_{1}$ & 40 \\
400 & $0.00250 \lambda_{1}$ & 44 \\
667 & $0.00150 \lambda_{1}$ & 47 \\
800 & $0.00125 \lambda_{1}$ & 48 \\
1000 & $0.00100 \lambda_{1}$ & 51 \\
\hline
\end{tabular}




\section{CHAPTER VI}

\section{CONCLUSION AND DISCUSSION}

\subsection{Summary of this Work}

In this thesis, first a mathematical formulation is introduced for the forward problem of induced current EIT and two numerical solution methods (SEM and FEM) are provided for its solution. Thereafter, the inverse problem is formulated and images are obtained using simulated data. The sensitivity of measurements to pixel conductivities, and ill-posedness of the inverse problem are investigated in detail. An alternative coil configuration is suggested to improve the performance of induced current EIT. A PC based data acquisition system is realized and images reconstructed by real data are presented.

\subsection{Conclusions reached}

In this thesis, it is shown that, induced current EIT is a feasible and promising technique for imaging 2-D conductivity distributions. However, an important research area in induced current EIT remains to be the design of a coil configuration (and the associated primary magnetic field) for which the imaging system parameters such as the number of independent measurements, the condition number of the sensitivity matrix, decay behaviour of the singular values and the sensitivity to inner region conductivity perturbation are optimized. It is interesting to note that often increasing relative sensitivity to the inner region is accompanied by decreasing condition number of the sensitivity matrix, in EIT in general.

The importance of the coil configuration is shown using even the 
circular coils for current induction by changing the amount of shift between the object center and the coil centers. It is shown that if this shift is decreased, the condition number is worsened (Figure 4.2 and 4.4 ) but the sensitivity to the inner region is somewhat increased (Figure 4.7). In fact, it is observed that the condition number decreases if the coils pass as close to the object as possible. Increasing radii of the coils also decreases the condition number but beyond a certain radius, the effect is negligible. In this study, the coils are taken to pass $0.01 \mathrm{~m}$ away from the object, and radii are taken to be $0.36 \mathrm{~m}$, this selection is found to be a good compromise for a $0.12 \mathrm{~m}$ radius object. A new coil configuration is suggested to improve the performance of induced current EIT. It is shown that, even using this intuitavely designed coil configuration, it is possible to introduce relatively large currents in the central region and hence increase the sensitivity of measurements to object's interior.

In this thesis, mainly the induced current EIT system with 9 circular current carrying coils is compared to the injected current EIT system using Sheffield data collection protocol and in general similar spatial resolutions were obtained. In order to be more complete in comparing induced and injected current EIT, performances of induced current EIT systems with different external coil configurations and of other injected current EIT systems whose current injection and data collection schemes are different from the Sheffield system must be considered [46], [34], [61]. However, a clear advantage of induced current EIT is that number of independent measurements can be increased by additional coils while in injected current EIT this can be achieved only by attaching additional electrodes. Of course, increasing the number of independent measurements is only useful if the number of normalized singular values which are larger than $1 /(\mathrm{SNR})$ is increased.

A prototype $\mathrm{PC}$ based data acquisition hardware is realized to show the feasiblity of induced current EIT. It is shown that, conventional data acquisition hardware can be used for data collection by addition of a simple coil drive electronics. The experimental results show that, the measurements are accurate enough to provide good estimates of actual object distributions. However, in order to collect more accurate data and hence reconstruct better images of actual objects, a new data acquisition hardware has to be developed. 
Although only 2-D problems are treated in this study, the derived equations can also be used to deal with 3-D problems. Since in 3-D problems FEM is computationally expensive, only 2-D problems are investigated in this thesis. However, actual objects are 3-D. In order to understand the sensitivity of measurements to off-plane conductivity perturbations and hence develop an image reconstruction algorithm, a 3-D FEM formulation is necessary. 


\section{REFERENCES}

[1] C. Altan, and Y. Z. İder, "Elektriksel Empedans Görüntülemesine Illişkin Bilgisayar Kontrollü Donanım", EMO Ulus. Kong. Kitapcı̆̆ı, 394, (1987).

[2] E. Atalar, and Y.Z. İder, "Tekrarlamalı Geri İzdüşüm Yöntemi ile Elektriksel Empedans Görüntülemesi”, EMO Ulus. Kong. Kitapcı̆̆ı, 398, (1987).

[3] E. Atalar, "An Iterative Backprojection Algorithm for Electrical Impedance Imaging using Finite Element Method", M. Sc. Thesis in EE Eng, METU, (July, 1987)

[4] D. C. Barber, B. H. Brown, and I. L. Freeston, "Imaging Spatial Distributions of Resistivity using Applied Potential Tomography", Electronic letters, $19,933,(1983)$.

[5] D. C. Barber, and B. H. Brown, "Applied Potential Tomography", J. Phys. E:Sci. Instrum., 17, 723, (1984).

[6] R. H. T. Bates, G. C. McKinnon, and A. D. Seagar, "A Limitation on Systems for Imaging Electrical Conductivity Distributions", IEEE Trans. Biomed. Eng., 27, 418, (1980).

[7] W. R. Breckon, and M. K. Pidcock, "Mathematical Aspects of Impedance Imaging", Clin. Phys. Physiol. Meas., $\underline{8}$, Supp. A, 77, (1987).

[8] W. R. Breckon, and M. K. Pidcock, "Ill-posedness and non-linearity in Electrical Impedance Tomography", Inform. Proc. Med. Imag., eds. C.N. Grauf and M. A. Wiergever, Plenum, New York, 235, (1988).

[9] W. R. Breckon, "Image Reconstruction in Electrical Impedance Tomography", Ph. D Thesis, School of Comp. Math. Sci., Oxford Polytechnic, (1990).

[10] B. H. Brown, and A. D. Seagar, "The Sheffield Data Collection System", Clin. Phys. Physiol. Meas., $\underline{8}, 91$, (1987). 
[11] B. H. Brown, D. C. Barber, and A. D. Seagar," Applied Potential Tomography: Possible Clinical Applications", Clin. Phys. Physiol. Meas., $\underline{6}, 109$, (1985).

[12] A. P. Calderon, "An Inverse Boundary Value Problem", Seminar on $\mathrm{Nu}$ merical Analysis and Quantum Mechanics, Rio de Janerio, 65, (1980).

[13] J. Conway," Electrical Impedance Tomography for thermal monitoring of hyperthermia treatment: an assesment using in vitro and in vivo measurements", Clin. Phys. Physiol. Meas., $\underline{8}$ Supp. A., 141, (1987).

[14] K. A. Dines, and R. J. Lytle," Analysis of Electrical Conductivity Imaging", $\underline{46}$, Geophysics, 1025, (1981).

[15] E. Dorken, H. Köymen, "Elektriksel Empedans Tomografisi Tanim Kumesinin Ultrasonik Yontemlerle Belirlenmesi", EMO Ulus. Kong. Kitapcigi, 387, (1987).

[16] E. Dorken, "Determination of Anatomical Boundaries by Ultrasound", M.Sc. Thesis in EE. Eng., METU , (June, 1988).

[17] B. M. Eyüboğlu, B. H. Brown, D. C. Barber, Seagar A. D., 1987, "Localization of Cardiac Related Impedance Changes in the Thorax", Clin. Phys. Physiol. Meas., $\underline{8}$, Supp. A., 167, (1987).

[18] B. M. Eyüboğlu, "Development and Application of Electrical Impedance Tomography for Thoracic Imaging", Ph D. Thesis in Dept. Med. Phys. and Clin. Eng., University of Sheffield, (1988).

[19] N. G. Gençer, and Y. Z. İder, "Empedans Görüntülemesinde Cebirsel Yöntemler”, EMO Ulus. Kong. Kitapcığı, 402, (1987).

[20] N. G. Gençer, "Study of Algebraic Reconstruction Algorithms for Practical Applications of EII", M.Sc. Thesis in EE Eng., METU, (1988).

[21] N. G. Gençer, and Y. Z. İder, "Genel Kesitli, Keside Dik Yönlerde İletkenliği Degişmeyen Yapılar için Elektriksel Empedans Tomografisi", ODTU EEMB 30. Yıl Sempozyumu, 103, (1989).

[22] N. G. Gençer, and Y. Z. İder, "A simulation study of the Resolution Limits of Electrical Impedance Tomography", $5^{\text {th }}$ Mediterrenean Conf. on Eng. Med. Biol., 356, (1989). 
[23] N. G. Gençer, B. Nakiboğlu, and Y. Z. İder, "Use of a Peripheral Layer of Known Conductivity for Electrical Impedance Tomography", Proc. CAIT Meeting on Electrical Impedance Tomography (Copenhagen), 76, (1991).

[24] N. G. Gençer, Y. Z. İder, and M. Kuzuoğlu, " Electrical Impedance Tomography using Induced and Injected Currents", Clin. Phys. Physiol. Meas., $\underline{13}$, Supp. A., 95, (1992).

[25] N. G. Gençer, Y. Z. İder, and B. Nakiboğlu, " An Alternative Solution for the Problem of Electrode Position Determination in Electrical Impedance Tomography", IEEE-EMBS $12^{\text {th }}$ Ann. Int. Conf. Proc., 128, (1990).

[26] N. G. Gençer, Y. Z. İder, and M. Kuzuoğlu, "Sensitivity Analysis and Inverse Problem Solution of Electrical Impedance Tomography Using Induced and Injected Currents", IEEE-EMBS $13^{\text {th }}$ Ann. Int. Conf. Proc., (1991).

[27] N. G. Gençer, Y. Z. İder, and M. Kuzuoğlu, "Sensitivity Matrix Analysis of the Back-Projection Algorithm in Electrical Impedance Tomography", IEEE-EMBS $14^{\text {th }}$ Ann. Int. Conf. Proc., 1682, (1992).

[28] N. G. Gençer, and Y. Z. İder, "An Experimental Study for Electrical Impedance Tomography Using Induced Currents", IEEE-EMBS $15^{\text {th }}$ Ann. Int. Conf. Proc., (1993), (submitted).

[29] N. G. Gençer, and Y. Z. İder, "Electrical Impedance Tomography Using Induced Currents : A Theoretical and Experimental Study", IEEE Trans. Nuc. Sci. Med. Imaging Conf., (1993), (submitted).

[30] N. G. Gençer, M. Kuzuoğlu, and Y. Z. İder, "Electrical Impedance Tomography Using Induced Currents", IEEE Trans. Med. Imag., (submitted on October 1992, revised on June 1993).

[31] D. B. Geselowitz, "An Application of Electricardiographic Lead Theory to Impedance Plethysmography", IEEE Trans. Biomed. Eng., 18, 38, (1971).

[32] H. Griffiths, A. Ahmed, "Applied Potential Tomography for Non-invasive Temperature Mapping in Hyperthermia", Clin. Phys. Physiol. Meas., $\underline{8}$, Supp A., 147, (1987). 
[33] D. G. Gisser, D. Isaacson, and J. C. Newel, "Theory and Performance of an Adaptive Current Tomography", Clin. Phys. Physiol. Meas., 9, Supp A., $35,(1988)$.

[34] D. G. Gisser, D. Isaacson, and J. C. Newel, "Electric Current Computed Tomography and Eigenvalues", Siam J. Appl. Math., $\underline{50}, 1623,(1990)$.

[35] G. H. Golub, and C. Reinsch, "Singular value decomposition and least squares solutions," Numer. Math., 14, 403, (1970).

[36] G. H. Golub, and C. F. Van Loan, Matrix computations, Baltimore: The Johns Hopkins University Press, 16, (1983).

[37] N. D. Harris, A. J. Sugget, D. C. Barber, and B. H. Brown, " Applications of Applied Potential Tomography (APT) in respiratory medicine", Clin. Phys. Physiol. Meas., $\underline{8}$, Supp. A., 155, (1987).

[38] T. J. Healey, R. C. Tozer, and I. L. Freeston., " Impedance Imaging of 3D Objects Using Magnetically Induced Currents", 14 ${ }^{\text {th }}$ IEEE-EMBS Ann. Int. Conf. Proc., 1719, (1993).

[39] R. P. Henderson, and J. G. Webster, "An Impedance Camera for Spatially Specific Measurements of The Thorax", IEEE Trans. Biomed. Eng., 25, $250,(1978)$.

[40] G. T. Herman, Image Reconstruction From Projections, The Fundementals of Computerized Tomography, Academic Press, New York, (1980).

[41] Y. Z. İder, C. Altan, E. Atalar, and N. G. Gençer, " Electrical Impedance Imaging System Applicable to Objects of Arbitrary but Known Boundary", $9^{\text {th }}$ IEEE-EMBS Ann. Int. Con. Proc., 1427, (1987).

[42] Y. Z. İder, E. Dorken, N. G. Gençer,and H. Köymen, " A Dual Modality Imaging System for Impedance Tomography with Ultrasonically Determined Boundaries", $10^{\text {th }}$ IEEE-EMBS Ann. Int. Conf. Proc., 283, (1988).

[43] Y. Z. İder, N. G. Gençer, "An Algorithm for Compensating 3-D Effects in Electrical Impedance Tomography", $11^{\text {th }}$ IEEE-EMBS Ann. Int. Conf. Proc., 465, (1989). 
[44] Y. Z. İder, B. Nakiboğlu, M. Kuzuoğlu, and N. G. Gençer, "Determination of the Boundary of an Object inserted into a Water filled Cylinder", Clin. Phys. Physiol. Meas., 13, Supp. A, 151, (1992).

[45] Y. Z. İder, N. G. Gençer, E. Atalar, and H. Tosun," Electrical Impedance Tomography of Translationaly Uniform Cylindrical Objects with general Cross sectional Boundaries", IEEE Trans. Medical Imaging, 37, 624, (1990).

[46] D. Isaacson, "Distinquishability of conductivities by electric current computed tomography", IEEE Trans. Med. Imaging, $\underline{5}, 91,(1986)$.

[47] J. Jossinet, and G. Kardous, "Physical Study of Sensitivity distribution in Multi-electrode systems", Clin. Phys. Physiol. Meas., $\underline{8}$, supp A, 33, (1987).

[48] E. C. Jordan, and K. G. Balmain, Electromagnetic Waves and Radiating Systems, New Jersey: Prentice-Hall Inc. 2nd Edition, 315, (1968).

[49] C. A. Kak, M. Slaney, Principles of Computerized Tomographic Imaging, IEEE Press, New York, (1985).

[50] Y. Kim, J. G. Webster, and W. J. Tompkins, "Electrical Impedance Imaging of the Thorax", J. Microwave Power, $\underline{8}$, pp 245, (1983).

[51] R. V. Kohn, and M. Vogelius, "Determining the Conductivity by Boundary Measurements", Commun. Pure. Appl. Math., 37, 289, (1984).

[52] R. V. Kohn, and M. Vogelius, "Identification of an Unknown Conductivity by means of Measurements at the Boundary", SIAM-AMS Proc., 14, (1984).

[53] R. V. Kohn, and M. Vogelius, "Determining the Conductivity by Boundary Measurements II, Interior Results", Commun. Pure. Appl. Math., 38, 644, (1985).

[54] E. E. Kriezis, T. D. Tsiobukis, S. M. Panas, and J. A. Tegopoulos, "Eddy Currents : Theory and applications", Proc. IEEE, $\underline{80}, 1559,(1992)$.

[55] M. Kuzuoğlu , K. Leblebicioğlu, N. G. Gençer, and Y. Z. İder, "A New Descent Algorithm for Electrical Impedance Tomography", IEEE-EMBS $14^{\text {th }}$ Ann. Int. Conf. Proc., 1684, (1992) 
[56] A. Macowski, Medical Imaging Systems, Prentice-Hall Inc., Englewood Cliffs, New Jersey, (1983).

[57] Y. F. Mangnall, A. J. Baxter, R. Avill, N. C. Bird, B. H. Brown, D. C. Barber, A. D. Seagar, A. G. Johnson, N. W. Read," Applied Potential Tomography: A New Non-invasive Technique for Assessing Gastric Function", Clin. Phys. Physiol. Meas., $\underline{8}$, Supp A., 119, (1987).

[58] T. Murai, Y. Kagawa, "Electrical Impedance Computed Tomography based on Finite Element Model", IEEE Trans. Biomed. Eng., 32, 177, (1985).

[59] D. Murphy, P. Burton, R. Coombs, L. Tarassenko, D. Rolfe, "Impedance Imaging in the new born", Clin. Phys. Physiol. Meas., ‥ Supp A., 131, (1987).

[60] B. Nakiboğlu, "Use of a Peripheral Water Layer of Known Conductivity for Electrical Impedance Tomography", M.Sc. Thesis in EE. Eng., METU., (1991).

[61] J. C. Newell, D. G. Gisser, and D. Isaacson, "An Electric Current Tomograph", IEEE Trans on Biomed. Eng., $\underline{35}, 828$, (1988).

[62] R. Plonsey, and R. E. Collin, Principles and Applications of Electromagnetic Fields, New York: McGraw-Hill Book Company, (1961).

[63] H. M. Powell, D. C. Barber, and I. L. Freeston, " Impedance Imaging using Linear Electrode Arrays", Clin. Phys. Physiol. Meas., $\underline{8}$, Supp. A, 109, (1987)

[64] L. R. Price, " Electrical Impedance Computed Tomography (ICT) : A New CT Imaging Technique", IEEE Trans. Nuc. Sci., 20, 2736, (1979)

[65] W. R. Purwis, R. C. Tozer, and I. L. Freeston, "Impedance Imaging Using Induced Currents", $12^{\text {th }}$ IEEE-EMBS Ann. Int. Conf. Proc., 114, (1990)

[66] J. Rosell, D. Murphy, R. Pallas, P. Rolfe, "Analysis and Assessment of Errors in a Parallel Data Acquisition System for Electrical Impedance Tomography", Clin. Phys. Physiol. Meas., 9, Supp. A, 93, (1988)

[67] E. Schomberg, and M. Tasto," Reconstruction of Spatial Resistivity Distribution from Resistance Projections", Philips gmbH Forschungslaboratorium, Hamburg, Report 2715/81, (1981). 
[68] J. M. Scaife, R. C. Tozer, and I. L. Freeston, "Real and imaginary impedance images using induced currents," Proc. $12^{\text {th }}$ Ann. Int. Conf. IEEE Eng. Med. Biol. Soc., 116, (1990).

[69] A. D. Seagar, D. C. Barber, and B. H. Brown," Theoretical Limits to Sensitivity and Resolution in Impedance Imaging", Clin. Phys. Physiol. Meas., $\underline{8}$, Supp. A, 13, (1987).

[70] A. D. Seagar, and B. H. Brown," Limitations in Hardware Design in Impedance Imaging", Clin. Phys. Physiol. Meas., $\underline{8}$, Supp. A, 85, (1987).

[71] P. P. Silvester, and R. L. Ferrari, Finite Elements for Electrical Engineers Cambridge University Press, (1983).

[72] A. M. Sinton, B. H. Brown, D. C. Barber, F. T. McArdle, and A. D. Leathard, "Noise and spatial Resolution of A Real Time Electrical Impedance Tomograph", Clin. Phys. Physiol. Meas., 13, Supp. A, 125, (1992).

[73] R. W. M. Smith, B. H. Brown, I. L. Freeston, and F. T. McArdle, "Real Time Electrical Impedance Tomography", Proc. CAIT Meeting on Electrical Impedance Tomograph (Copenhagen), 206, (1990).

[74] R. W. M. Smith, B. H. Brown, I. L. Freeston, F. T. McArdle, D. C. Barber, "Real Time Electrical Impedance Imaging", Proc. $12^{\text {th }}$ Int. Conf. IEEEEMBS, 104, (1990).

[75] J. Sylvester, and G. Uhlman, " A Uniqueness theorem for an Inverse Boundary Value Problem in Electrical Prospection", Commun. Pure. Appl. Math., 39, 91, (1986)

[76] L. Tarassenko, P. Rolfe, " Imaging Spatial Distributions of Resistivity, an alternative approach", Electronic Letters, 20, 574, (1984).

[77] C. Trillaud, and J. Jossinet, "An Improved design of Voltmeter for Semi Parallel Data Acquisition ", Clin. Phys. Physiol. Meas., 13, Supp. A, 5, (1992).

[78] S. Webb, The Physics of Medical Imaging, IOP Publishing Ltd., Bristol, (1988). 
[79] A. Wexler, B. Fry, and M. R. Neuman, " Impedance Computed Tomography algorithm and System", Appl. Opt., $\underline{24}, 3985,(1985)$.

[80] T. J. Yorkey, J. G. Webster, " A Comparison of Impedance reconstruction algorithms", Clin. Phys. Physiol. Meas., $\underline{8}$, Supp. A, 55, (1987). 
APPENDICES 


\section{APPENDIX A}

\section{CALCULATION OF $\vec{A}$}

Let $\left(x^{\prime}, y^{\prime}\right)$ be the source point on a coil configuration and $(x, y)$ be the observation point, the solution for the magnetic vector potential $\vec{A}$ is

$$
\vec{A}(x, y)=\frac{\mu_{0}}{4 \pi} \int_{S} \frac{\vec{J}\left(x^{\prime}, y^{\prime}\right)}{R} e^{-j k R} d S^{\prime}
$$

where $R=\left[\left(x-x^{\prime}\right)^{2}+\left(y-y^{\prime}\right)^{2}\right]^{\frac{1}{2}}, k^{2}=\omega^{2} \mu_{0} \epsilon$ and $\mu_{0}$ and $\epsilon$ is the permeability and permittivity, respectively and $d S^{\prime}$ represents the surface element on the source region $S$ [62]. However, since the immediate vicinity of sources (inside the coil) are of interest, and the extent of the source region (coil diameter is less than 1 meter) is small compared with a wavelength $\lambda(\lambda=6000 \mathrm{~m}$ for $50 \mathrm{kHz}$ excitation frequency), then $k R=\frac{2 \pi R}{\lambda}$ is very small. Therefore the exponential term $e^{-j k R}$ can be replaced by unity and the solution for magnetic vector potential reduces to

$$
\vec{A}(x, y)=\frac{\mu_{0}}{4 \pi} \int_{S} \frac{\vec{J}\left(x^{\prime}, y^{\prime}\right)}{R} d S^{\prime}
$$

For filamentary currents $\vec{A}$ can be obtained by

$$
\vec{A}(x, y)=\frac{\mu_{0} I}{4 \pi} \oint_{C} \frac{d \vec{l}}{R}
$$

where $I$ represents the current in the filamentary wire represented by the closed curve $C$ and $d \vec{l}$ denotes the differential element on this curve.

In this study, the integration given in the above equation is approximated numerically for the $x$ and $y$ components of $\vec{A}$ by

$$
\begin{gathered}
A_{x}=\frac{\mu_{0}}{4 \pi} \sum_{n=1}^{N} \frac{-\operatorname{Sin}\left(n \frac{2 \pi}{N}\right)}{R} a \frac{2 \pi}{N} \\
A_{y}=\frac{\mu_{0}}{4 \pi} \sum_{n=1}^{N} \frac{\operatorname{Cos}\left(n \frac{2 \pi}{N}\right)}{R} a \frac{2 \pi}{N}
\end{gathered}
$$


where $N$ denotes the number of integration points, $a$ is the coil radius and $\mu_{0}$ is the free space permeability. Note that the divergence of $\vec{A}$ given by equation (A.3) is zero. The proof of this identity can be found in [62]. 


\section{APPENDIX B}

\section{FEM FORMULATION}

Let a rectangular object be divided into two triangular regions of constant conductivities $\sigma_{1}$ and $\sigma_{2}$ as shown in Fig B.1. Using Galerkin weighted residuals method, the scalar potential equation (2.21) with (2.22) is to be satisfied on each element by multiplying both sides with an appropriate shape function and integrating over the element area $S$ [71]. For example, for the first element this yields

$$
\int_{S_{1}} N_{1}^{1} \nabla \cdot\left(\sigma_{1} \nabla \phi\right) d S=-\omega \int_{S_{1}} N_{1}^{1} \nabla \sigma \cdot \vec{A} d S
$$

for $i$ and $j=1 \cdots 3$, where $N_{i}^{j}$ is the $i^{\text {th }}$ shape function for the $j^{\text {th }}$ element. However, since $\sigma$ is constant on each element, the right hand side of equation (B.1) is zero yielding the following equation after using appropriate vector identities and the Gauss' theorem,

$$
\int_{S_{1}} \sigma_{1} \nabla N_{1}^{1} \cdot \nabla \phi d S=\oint_{\Gamma_{1}} N_{1}^{1} \sigma_{1} \nabla \phi \cdot \vec{n} d l
$$

where $\vec{n}$ is the outward normal on each side of the element and $\Gamma_{1}$ denotes the contour along the boundary of the first element. Applying the same procedure by using other shape functions $N_{2}^{j}$ and $N_{3}^{j}$, then for each element $\mathrm{j}$, the following element matrix equation has to be satisfied.

$$
\sigma_{j}\left[\begin{array}{ccc}
s_{11}^{j} & s_{12}^{j} & s_{13}^{j} \\
s_{21}^{j} & s_{22}^{j} & s_{23}^{j} \\
s_{31}^{j} & s_{32}^{j} & s_{33}^{j}
\end{array}\right]\left[\begin{array}{c}
\phi_{1}^{j} \\
\phi_{2}^{j} \\
\phi_{3}^{j}
\end{array}\right]=\left[\begin{array}{c}
\oint_{\Gamma_{1}} N_{1}^{j} \sigma_{j} \nabla \phi \cdot \vec{n} d l \\
\oint_{\Gamma_{1}} N_{2}^{j} \sigma_{j} \nabla \phi \cdot \vec{n} d l \\
\oint_{\Gamma_{1}} N_{3}^{j} \dot{\sigma}_{j} \nabla \phi \cdot \vec{n} d l
\end{array}\right]
$$


where the expressions for $s_{k l}^{j}$ can be found elsewhere [71]. The resultant system of equations using the global node numbering will be

$$
\left[\begin{array}{llll}
\sigma_{1} s_{11}^{1} & \sigma_{1} s_{12}^{1} & 0 & \sigma_{1} s_{13}^{1} \\
\sigma_{1} s_{21}^{1} & \sigma_{1} s_{22}^{1}+\sigma_{2} s_{11}^{2} & \sigma_{2} s_{13}^{2} & \sigma_{1} s_{23}^{1}+\sigma_{2} s_{12}^{2} \\
0 & \sigma_{2} s_{31}^{2} & \sigma_{2} s_{33}^{2} & \sigma_{2} s_{32}^{2} \\
\sigma_{1} s_{31}^{1} & \sigma_{1} s_{32}^{1}+\sigma_{2} s_{22}^{2} & \sigma_{2} s_{23}^{2} & \sigma_{1} s_{33}^{1}+\sigma_{2} s_{23}^{2}
\end{array}\right]\left[\begin{array}{l}
\phi_{1} \\
\phi_{2} \\
\phi_{3} \\
\phi_{4}
\end{array}\right]=b(\bar{\sigma})
$$

where $b(\bar{\sigma})$ is a $4 \times 1$ vector as

$$
\left[\begin{array}{l}
\int_{1}^{2} \sigma_{1} N_{1}^{1} \nabla \phi \cdot \vec{n} d l+\int_{4}^{1} \sigma_{1} N_{1}^{1} \nabla \phi \cdot \vec{n} d l \\
\int_{1}^{2} \sigma_{1} N_{2}^{1} \nabla \phi \cdot \vec{n} d l+\left(\int_{4}^{2} \sigma_{2} N_{1}^{2} \nabla \phi \cdot \vec{n} d l+\int_{2}^{4} \sigma_{1} N_{2}^{1} \nabla \phi \cdot \vec{n} d l\right)+\int_{2}^{3} \sigma_{2} N_{1}^{2} \nabla \phi \cdot \vec{n} d l \\
\int_{3}^{4} \sigma_{2} N_{3}^{2} \nabla \phi \cdot \vec{n} d l+\int_{2}^{3} \sigma_{2} N_{3}^{2} \nabla \phi \cdot \vec{n} d l \\
\int_{4}^{1} \sigma_{1} N_{3}^{1} \nabla \phi \cdot \vec{n} d l+\left(\int_{2}^{4} \sigma_{1} N_{3}^{1} \nabla \phi \cdot \vec{n} d l+\int_{4}^{2} \sigma_{2} N_{2}^{2} \nabla \phi \cdot \vec{n} d l\right)+\int_{3}^{4} \sigma_{2} N_{2}^{2} \nabla \phi \cdot \vec{n} d l
\end{array}\right]
$$

But for sides (1-2), (2-3), (3-4) and (1-4) the boundary condition specified by equation (2.22) and for the side (2-4) the interface condition (similar to the condition given by equation (3.9)) has to be applied. Using these conditions the right hand side of equation (B.4) reduces to

$$
-\omega\left[\begin{array}{l}
\int_{1}^{2} \sigma_{1} N_{1}^{1} A_{1 n} d l+\int_{4}^{1} \sigma_{1} N_{1}^{1} A_{1 n} d l \\
\int_{2}^{4} \sigma_{1} N_{2}^{1} A_{1 n} d l+\left(\int_{4}^{2} N_{1}^{2}\left(\sigma_{2} A_{2 n}-\sigma_{1} A_{1 n}\right) d l\right)+\int_{2}^{3} \sigma_{2} N_{1}^{2} A_{2 n} d l \\
\int_{3}^{4} \sigma_{2} N_{3}^{2} A_{2 n} d l+\int_{2}^{3} N_{3}^{2} A_{2 n} d l \\
\int_{4}^{1} \sigma_{1} N_{3}^{1} A_{1 n} d l+\left(\int_{2}^{4} N_{3}^{1}\left(\sigma_{1} A_{1 n}-\sigma_{2} A_{2 n}\right) d l\right)+\int_{3}^{4} \sigma_{2} N_{2}^{2} A_{2 n} d l
\end{array}\right]
$$

where $A_{\text {in }}$ denotes the normal component of magnetic vector potential $\vec{A}$ on the specified side of the $i^{\text {th }}$ element. $\vec{A}$ is calculated at the center of each element and assumed to be constant on element area.

Equation (B.4) can be expressed in matrix notation as

$$
S(\bar{\sigma}) \bar{V}=b(\bar{\sigma})
$$

where $S$ denotes the $4 \times 4$ coefficient matrix, $\bar{V}$ is the $4 \times 1$ vector of nodal scalar potentials, and $\bar{\sigma}$ is $2 \times 1$ vector of element conductivities. 


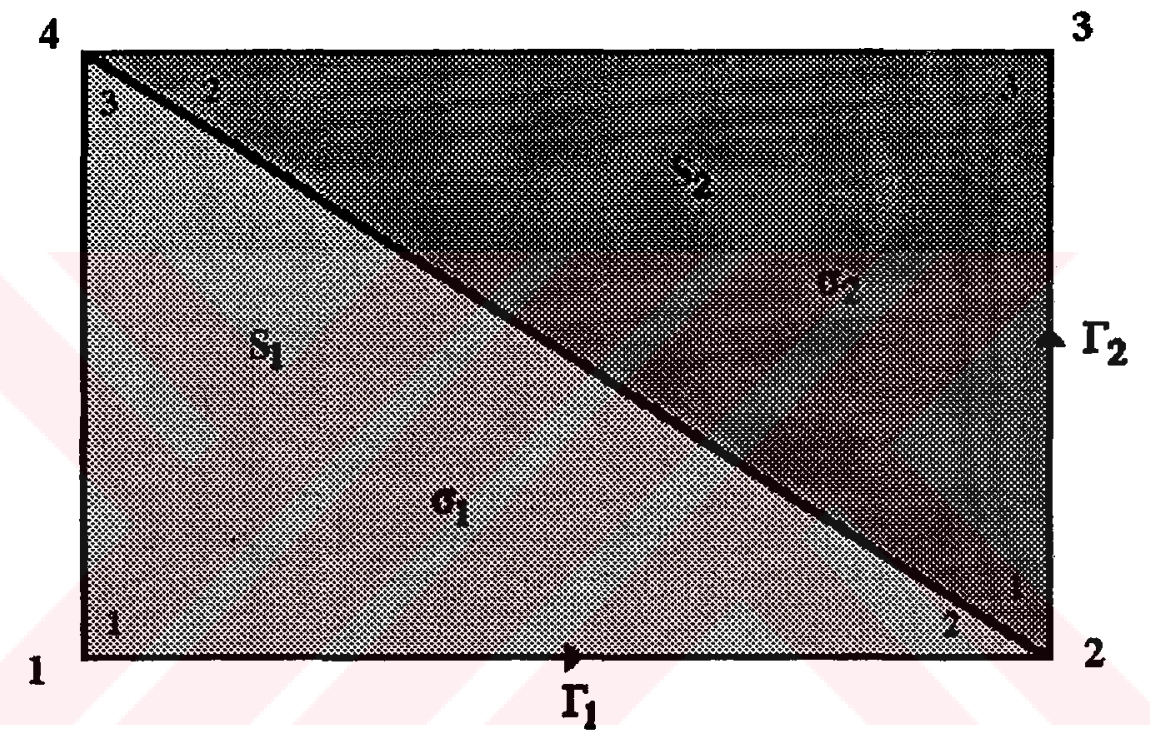

Figure B.1. A mesh with 4 nodes and 2 triangular elements. The numbers inside the elements represent the local node numbering whereas the numbers outside the rectangular region are the global numbers of the nodes. $\Gamma_{1}$ and $\Gamma_{2}$ denote the counter clockwise contours on the boundary of the elements. $S_{1}, S_{2}$ and $\sigma_{1}, \sigma_{2}$ are the element areas and conductivities, respectively. 


\section{APPENDIX C}

\section{VALIDATION OF THE ASSUMPTIONS}

\section{C.1 Introduction}

In the forward problem formulation, two assumptions are proposed to simplify the problem of finding the scalar potential function in $\Omega$. Equations (2.11) and (2.13) derived in section 2.2 are the coupled partial differential equations whose solution for $\phi$ and $\vec{A}$ is a difficult task even for 2-D problems. However, in that section, the total magnetic vector potential $\vec{A}$ is assumed to be approximately equal to the primary magnetic vector potential $A_{p}$. This assumption makes the solution of equation (2.13) unnecessary since $\overrightarrow{A_{p}}$ is a quantity that can be evaluated as it is explained in Appendix A. The solution of $\phi$ is greatly simplified since now $\vec{A}_{p}$ becomes the known source of equation (2.11). The validation of this assumption requires to show that the secondary magnetic vector potential (caused by the induced currents in the conductive medium) is negligible with respect to $\vec{A}_{p}$. The second assumption is based on the idea that the conduction and displacement currents behave as sources of the imaginary and real parts of the potential distribution, respectively. For conductivity imaging, neglection of the displacement currents in equation (2.11) will not change the solution of $\phi_{I}$ if the assumption holds. The validation of this assumption requires the solution of $\phi$ with and without the displacement currents and check whether there is a significant difference in $\phi_{1}$ in both cases.

In the first part of this section, the validity of the first assumption will be tested by first formulating the partial differential equation for $\overrightarrow{A_{s}}$ using equation (2.14) (the displacement currents are assumed to be negligible). Then, $\overrightarrow{A_{s}}$ is evaluated using a first order approximation for different conductivity distributions. In the second part, a new formulation for SEM will be used to solve 
the scalar potential in objects of circular cross sections with two concentric layers having different conductivity and permittivity distributions. The imaginary part of scalar potential gradients will be compared with the solutions provided by SEM using equations (3.6) and (3.7).

\section{C.2 Validation of the First Assumption}

In equation (2.14), $\vec{A}$ represents the total magnetic vector potential. The relation of $\vec{A}$ with the secondary $\left(\overrightarrow{A_{p}}\right)$ and primary $\left(\overrightarrow{A_{s}}\right)$ magnetic vector potentials can be expressed as

$$
\vec{A}=\overrightarrow{A_{p}}+\overrightarrow{A_{s}}
$$

Using the above expression for $\vec{A}$ and omitting the displacement currents, equation (2.14) can be rewritten as

$$
\nabla^{2} \overrightarrow{A_{s}}=\mu_{0} \sigma\left(-\nabla \phi-\omega \overrightarrow{A_{p}}-\omega \overrightarrow{A_{s}}\right)
$$

The first order approximation for $\overrightarrow{A_{s}}$ can be obtained by neglecting the $\overrightarrow{A_{s}}$ term in the right hand side and reducing equation (C.2) to

$$
\nabla^{2} \overrightarrow{A_{s}}=\mu_{0} \sigma\left(-\nabla \phi-\omega \overrightarrow{A_{p}}\right)
$$

or by decomposing the terms into their real and imaginary parts

$$
\begin{aligned}
\nabla^{2} \overrightarrow{A_{s R}} & =-\mu_{0} \sigma \nabla \phi_{R} \\
\nabla^{2} \overrightarrow{A_{s I}} & =\mu_{0} \sigma\left(-\nabla \phi_{I}-\omega \overrightarrow{A_{p}}\right)
\end{aligned}
$$

Since $\phi$ is solved by neglecting $\overrightarrow{A_{s}}, \phi_{R}$ is zero as it is shown in Section 2.2 by equations (2.17) with (2.18). Therefore, the right hand side of equation (C.4) becomes zero. Equation (C.5) can be expressed as

$$
\nabla^{2} \overrightarrow{A_{s I}}=\mu_{0} \vec{J}
$$

where $\vec{J}$ is the induced conduction current in the medium. The real part of $\overrightarrow{A_{s}}$ is zero since the right hand side of the related partial differential equation (C.4) is zero. The value of $\overrightarrow{A_{s I}}$ at a point $P$ in $\Omega$ can be obtained by

$$
\overrightarrow{A_{s I}}(P)=-\frac{\mu_{0}}{4 \pi} \int_{\Omega} \frac{\vec{J}}{R} d \Omega
$$


where $R$ represents the distance from the area element $d \Omega$ to the point $P$ in $\Omega$ [62]. The solution of $\overrightarrow{A_{s} I}$ is approximated by making use of FEM tools developed in this study. The solution of the secondary magnetic vector potential on the $k^{\prime} t h$ element is calculated as

$$
\overrightarrow{A_{s I}}(k)=-\frac{\mu_{0}}{4 \pi} \sum_{i=1}^{1016} \frac{\vec{J}_{i}}{R_{i k}} S_{i}+\frac{1}{2} \mu_{0} \vec{J}_{k} a_{k}
$$

where $R_{i k}$ is the distance between the $i^{t h}$ and the $k^{t h}$ element, $S_{i}$ denotes the area of the $i^{t h}$ element and $\vec{J}_{i}$ is the current density on the $i^{\text {th }}$ element. The second term in the right hand side represents the contribution of the current density on the $k^{\text {th }}$ element. This term is obtained by assuming a circular region of area $S_{k}$ with center located in the $k^{\text {th }}$ triangular element. The radius of the circular region is denoted by $a_{k}$ and calculated as $a_{k}=\sqrt{S_{k} / \pi}$. The element currents are calculated for a coil of radius $0.36 \mathrm{~m}$ and shifted $0.20 \mathrm{~m}$ from the origin of $\Omega$ along the $x$ axis. Two cases are treated, namely the solution of $\overrightarrow{A_{s}}$ for the uniform object $(0.2$ Siemens $/ \mathrm{m}$ ) and for the non-uniform object (alpha= $10, \beta=0.33, \sigma_{2}=0.2$ Siemens $/ m$ ). The magnitudes of $\omega \overrightarrow{A_{s}}$ are calculated for the elements located on the $x$ axis of the object region and given in Table C.1 for both cases. The corresponding $\omega \overrightarrow{A_{p}}$ values are also calculated on these elements and given in the last column of this table for comparison. Although equation (C.8) provides an approximate solution for $\vec{A}_{s}$, it gives sufficient information about the order of magnitude of $\vec{A}_{s}$ relative to $\vec{A}_{p}$. It is observed that for both uniform and non-uniform objects the magnitudes of $\overrightarrow{A_{s}}$ is much smaller than the corresponding ones calculated for $\overrightarrow{A_{p}}$ which shows the validity of the first assumption.

\section{C.3 Validation of the Second Assumption}

The following equations can be obtained from equation (2.11) by decomposing $\phi$ into its real and imaginary components.

$$
\begin{aligned}
\nabla \cdot\left(\sigma \nabla \phi_{R}\right)-\omega \nabla \cdot\left(\epsilon \nabla \phi_{I}\right) & =\omega^{2} \vec{A} \cdot \nabla \epsilon \\
\omega \nabla \cdot\left(\epsilon \nabla \phi_{R}\right)+\nabla \cdot\left(\sigma \nabla \phi_{I}\right) & =-\omega \vec{A} \cdot \nabla \sigma
\end{aligned}
$$


Table C.1. Comparison of $\left|\omega \overrightarrow{A_{s}}\right|$ and $\left|\omega \overrightarrow{A_{p}}\right|$ in both uniform and non-uniform objects

\begin{tabular}{||c|c|c|c|c||}
\hline \multirow{2}{*}{$x(\mathrm{~cm})$} & & \multicolumn{2}{|c||}{$\mid \omega(\mathrm{cm})$} & $\left|\omega \vec{A}_{s}\right|$ \\
\cline { 3 - 4 } & & $\begin{array}{c}\text { uniform } \\
\text { object }(V / m)\end{array}$ & $\begin{array}{c}\text { non-uniform } \\
\text { object }(V / m)\end{array}$ & $(V / m)$ \\
\hline-11.45 & -0.29 & $6.64 \mathrm{e}-9$ & $7.07 \mathrm{e}-9$ & $8.53 \mathrm{e}-5$ \\
-8.25 & 0.29 & $6.34 \mathrm{e}-9$ & $7.13 \mathrm{e}-9$ & $8.50 \mathrm{e}-5$ \\
-4.24 & -0.13 & $3.00 \mathrm{e}-9$ & $6.28 \mathrm{e}-9$ & $8.44 \mathrm{e}-5$ \\
0.48 & 0.10 & $4.99 \mathrm{e}-10$ & $3.52 \mathrm{e}-10$ & $8.44 \mathrm{e}-5$ \\
3.68 & 0.00 & $3.64 \mathrm{e}-9$ & $9.44 \mathrm{e}-9$ & $8.43 \mathrm{e}-5$ \\
7.72 & 0.00 & $5.75 \mathrm{e}-9$ & $6.33 \mathrm{e}-9$ & $8.43 \mathrm{e}-5$ \\
11.45 & 0.29 & $5.10 \mathrm{e}-9$ & $5.32 \mathrm{e}-9$ & $8.47 \mathrm{e}-5$ \\
\hline
\end{tabular}

with the boundary conditions

$$
\begin{aligned}
& \frac{\partial \phi_{R}}{\partial n}=0 \\
& \frac{\partial \phi_{I}}{\partial n}=-\omega \vec{A} \cdot \vec{n}
\end{aligned}
$$

as given by equations (2.18) and (2.20). For the solution of $\phi$ using the equations given above, the first assumption is taken to be valid, i.e $\vec{A}$ represents the primary magnetic vector potential. The solutions are obtained by using SEM for 2-D circular objects with two concentric layers of different conductivity and permittivity. For the problem geometry given in Figure C.1, the expressions for $\phi$ given by equations (3.6) and (3.7) are still valid except that there are complex coefficients in the series. The necessary boundary and interface conditions are similar to the ones given by equations (3.8) through (3.10) but in this case the displacement currents are included. These conditions are :

$$
\begin{aligned}
\left.\frac{\partial \phi_{2}}{\partial r}\right|_{r=R_{2}} & =-j \omega A_{n}\left(R_{2}\right) \\
\left(\sigma_{2}+j \omega \epsilon_{2}\right)\left(\left.\frac{\partial \phi_{2}}{\partial r}\right|_{r=R_{1}}+j \omega A_{n}\left(R_{1}\right)\right) & = \\
\left(\sigma_{1}+j \omega \epsilon_{1}\right)\left(\left.\frac{\partial \phi_{1}}{\partial r}\right|_{r=R_{1}}+j \omega A_{n}\left(R_{1}\right)\right) & \\
\phi_{2}\left(R_{1}\right) & =\phi_{1}\left(R_{1}\right)
\end{aligned}
$$


The real and imaginary parts of the coefficients $a_{m}, b_{m}$ and $e_{m}$ can be found by solving the following equations :

$$
\begin{aligned}
R_{2}^{m} a_{m R}-R_{2}^{-m} b_{m R} & =0 \\
R_{2}^{m} a_{m I}-R_{2}^{-m} b_{m I} & =0 \\
\sigma_{2}\left(R_{1}^{m} a_{m R}-R_{1}^{-m} b_{m R}\right)-\sigma_{2} \alpha e_{m R} R_{1}^{m} & \\
-\omega \epsilon_{2}\left(R_{1}^{m} a_{m I}-R_{1}^{-m} b_{m I}\right)+\omega \epsilon_{2} \gamma e_{m I} R_{1}^{m} & =-\omega \epsilon_{2}(1-\gamma) R_{1} E(m) \\
\omega \epsilon_{2}\left(R_{1}^{m} a_{m R}-R_{1}^{-m} b_{m R}\right)-\omega \epsilon_{2} \gamma e_{m R} R_{1}^{m} & \\
+\sigma_{2}\left(R_{1}^{m} a_{m I}-R_{1}^{-m} b_{m I}\right)-\sigma_{2} \alpha e_{m I} R_{1}^{m} & =\sigma_{2}(1-\alpha) R_{1} E(m) \\
R_{1}^{m} a_{m R}+R_{1}^{-m} b_{m R} & =R_{1}^{m} e_{m R} \\
R_{1}^{m} a_{m I}-R_{1}^{-m} b_{m I} & =R_{1}^{m} e_{m I}
\end{aligned}
$$

where the subscripts $I_{I}$ and ${ }_{R}$ denote the imaginary and real components of the related parameters. The coefficients $c_{m}, d_{m}$, and $f_{m}$ satisfy the same type of equations but $a_{m}, b_{m}, e_{m}, C(m)$ and $E(m)$ has to be replaced by $c_{m}, d_{m}, f_{m}$, $D(m)$ and $F(m)$, respectively.

The solution of these equations yields the following expressions :

$$
\begin{gathered}
a_{m R}=\frac{1}{Q}\left\{\left[(\alpha-\gamma) \sigma_{2}\left(\omega \epsilon_{2}\right)\right] e_{m I}+\left[(1+\alpha) \sigma_{2}^{2}+(1+\gamma)\left(\omega \epsilon_{2}\right)^{2}\right] e_{m R}\right. \\
\left.-\left[(\alpha-\gamma) \sigma_{2}\left(\omega \epsilon_{2}\right)\right] \frac{1}{R_{1}^{m-1}} E(m)\right\} \\
a_{m I}=\frac{1}{Q}\left\{\left[(1+\alpha) \sigma_{2}^{2}+(1+\gamma)\left(\omega \epsilon_{2}\right)^{2}\right] e_{m I}-\left[(\alpha-\gamma) \sigma_{2}\left(\omega \epsilon_{2}\right)\right] e_{m R}\right. \\
\left.+\left[(1-\alpha) \sigma_{2}^{2}+(1-\gamma)\left(\omega \epsilon_{2}\right)^{2}\right] \frac{1}{R_{1}^{m-1}} E(m)\right\} \\
b_{m R}=-\frac{1}{Q}\left\{\left[(\alpha-\gamma) \sigma_{2}\left(\omega \epsilon_{2}\right)\right] R_{1}^{2 m} e_{m I}+\left[(1-\alpha) \sigma_{2}^{2}+(1-\gamma)\left(\omega \epsilon_{2}\right)^{2}\right] R_{1}^{2 m} e_{m R}\right. \\
\left.+\left[(\alpha-\gamma) \sigma_{2}\left(\omega \epsilon_{2}\right)\right] R_{1}^{m+1} E(m)\right\} \\
b_{m I}=\frac{1}{Q}\left\{\left[(1-\alpha) \sigma_{2}^{2}+(1-\gamma)\left(\omega \epsilon_{2}\right)^{2}\right] R_{1}^{2 m} e_{m I}+\left[(\alpha-\gamma) \sigma_{2}\left(\omega \epsilon_{2}\right)\right] R_{1}^{2 m} e_{m R}\right. \\
\left.-\left[(1-\alpha) \sigma_{2}^{2}+(1-\gamma)\left(\omega \epsilon_{2}\right)^{2}\right] R_{1}^{m+1} E(m)\right\} \\
e_{m R}=\left(a_{22} g_{1}-a_{12} g_{2}\right) / \text { det } \\
e_{m I}=\left(a_{11} g_{2}-a_{21} g_{1}\right) / \text { det } \\
d e t=a_{11} a_{22}-a_{12} a_{21}
\end{gathered}
$$

where

$$
a_{11}=R_{2}^{m}\left(1+\beta^{2 m}\right)(\alpha-\gamma) \sigma_{2}\left(\omega \epsilon_{2}\right)
$$




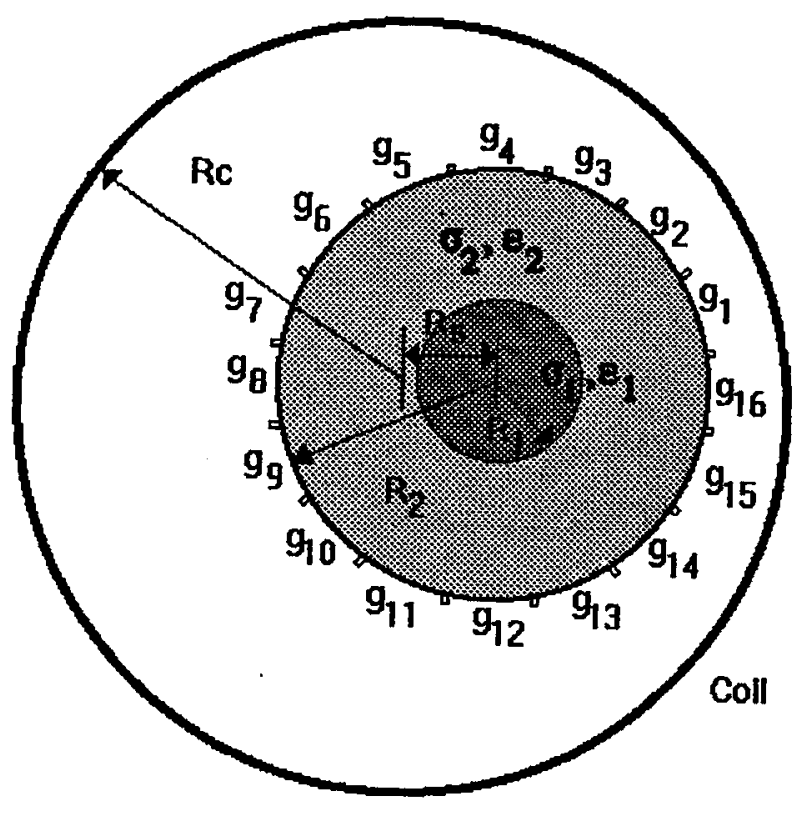

Figure C.1. The object distribution used for the solution of the scalar potential function including the effects of displacement currents. $\sigma_{1}, \epsilon_{1}$ and $\sigma_{2}, \epsilon_{2}$ represent the conductivity and permittivity of the central inhomogeneity and the peripheral, respectively.

$$
\begin{array}{rlr}
a_{12} & =R_{2}^{m}\left[(1+\alpha)-(1-\alpha) \beta^{2 m}\right] \sigma_{2}^{2}+\left[(1+\gamma)-(1-\gamma) \beta^{2 m}\right]\left(\omega \epsilon_{2}\right)^{2}(\mathrm{C} .30) \\
a_{21} & =a_{12} & \text { (C.31) } \\
a_{22} & =-a_{11} & \text { (C.32) } \\
\gamma & =\frac{\omega \epsilon_{1}}{\omega \epsilon_{2}} & \text { (C.33) } \\
Q & =2\left[\sigma_{2}^{2}+\left(\omega \epsilon_{2}\right)^{2}\right] & \text { (C.34) } \\
g_{1} & =\frac{R_{2}}{\beta^{m-1}} \sigma_{2}\left(\omega \epsilon_{2}\right)(\alpha-\gamma)\left(1+\beta^{2 m}\right) E(m) \\
g_{2} & =R_{2} Q C(m)-\frac{R_{2}}{\beta^{m-1}}\left[(1-\alpha) \sigma_{2}^{2}+(1-\gamma)\left(\omega \epsilon_{2}\right)^{2}\right]\left(1+\beta^{2 m}\right) E(m(\mathrm{C} .36)
\end{array}
$$

and $\alpha, \beta, C(m), D(m), E(m)$ and $F(m)$ are as defined before in Section 3.2. 


\section{C.4 Results}

The solution of the scalar potential is obtained using the series expansions given in equations (3.6) and (3.7) using the coefficient equations given by equations (C.22) through (C.36). The real and imaginary components of the boundary potential differences are calculated for both uniform and non-uniform objects and given in Table C.2. The definition of the uniform and non-uniform objects in this case are different from the usual definitions used throughout this study. Since the displacement currents are included, it is necessary to specify the permittivity distribution in $\Omega$. For the uniform object distribution, conductivity and permittivity in $\Omega$ are assumed to be 0.2 Siemens $/ \mathrm{m}$ and $75 \epsilon_{0}$, respectively where $\epsilon_{0}\left(=8.854 \times 10^{-12} \mathrm{Farad} / \mathrm{m}\right)$ represents the permittivity of free space. The non-uniform object is defined by the parameters $\alpha=0.03$, $\beta=0.33, \gamma=3.33$ where $\sigma_{2}=0.2$ Siemens $/ m$ and $\epsilon_{2}=75 \epsilon_{0}$. The conductivity and permittivity values of the peripheral layer and the central inhomogeneity simulate the properties of saline and bone [68], respectively. The imaginary part of the boundary potential differences are also calculated by using SEM equations (2.21) and (2.22) (derived by neglecting displacement currents) and given in Table C.2. It is observed that for uniform object distribution, the permittivity of the object does not affect the solution of the scalar potential, i.e., the real part of gradients is zero and imaginary part is equal to the gradients calculated by neglecting the displacement currents. The real part of the gradients is much smaller than the imaginary part for the non-uniform object distribution and the imaginary part is equal to the gradients calculated by neglecting the displacement currents up to 4 digits. The results are strongly encouraging to conclude that the diplacement currents can be excluded in the scalar potential formulations for conductivity imaging. 
Table C.2. Comparison of the boundary potential differences obtained by SEM to show the negligibility of displacement currents. The first two columns show the SEM solutions without displacement currents. In the later columns solutions of SEM are given when the displacement currents are included for both uniform and non-uniform object distributions. $\overline{g_{I}}$ and $\overline{g_{R}}$ represents imaginary and real parts of the gradients.

\begin{tabular}{|c|c|c|c|c|c|c|}
\hline \multirow{3}{*}{$\begin{array}{l}\text { Boundary } \\
\text { potential } \\
\text { differences }\end{array}$} & \multicolumn{2}{|c|}{$\begin{array}{l}\text { SEM solutions without } \\
\text { displacement currents }\end{array}$} & \multicolumn{4}{|c|}{$\begin{array}{l}\text { SEM solutions including } \\
\text { displacement currents }\end{array}$} \\
\hline & \multirow{2}{*}{$\begin{array}{c}\text { uniform } \\
\text { object } \\
\bar{g}_{I}(m V)\end{array}$} & \multirow{2}{*}{$\begin{array}{c}\text { non-uniform } \\
\text { object } \\
\bar{g}_{I}(m V) \\
\end{array}$} & \multicolumn{2}{|c|}{$\begin{array}{l}\text { uniform } \\
\text { object }\end{array}$} & \multicolumn{2}{|c|}{$\begin{array}{c}\text { non-uniform } \\
\text { object }\end{array}$} \\
\hline & & & $\overline{g_{R}}(\mu V)$ & $\bar{g}_{I}(m V)$ & $\overline{g_{R}}(\mu V)$ & $\bar{g}_{I}(m V)$ \\
\hline$g_{1}$ & 2.7205 & 2.770727 & 0.0000 & 2.7205 & 0.3879 & 2.770725 \\
\hline$g_{2}$ & 2.2253 & 2.264927 & 0.0000 & 2.2253 & 0.3046 & 2.264926 \\
\hline$g_{3}$ & 1.4245 & 1.447443 & 0.0000 & 1.4245 & 0.1754 & 1.447442 \\
\hline$g_{4}$ & 0.3600 & 0.362216 & 0.0000 & 0.3600 & 0.0153 & 0.362216 \\
\hline$g_{5}$ & -0.8922 & -0.912074 & 0.0000 & -0.8922 & -0.1537 & -0.912073 \\
\hline$g_{6}$ & -2.1880 & -2.227595 & 0.0000 & -2.1880 & -0.3046 & -2.227594 \\
\hline$g_{7}$ & -3.2523 & -3.305692 & 0.0000 & -3.2523 & -0.4096 & -3.305690 \\
\hline$g_{8}$ & -3.6833 & -3.741660 & 0.0000 & -3.6833 & -0.4473 & -3.741658 \\
\hline$g_{9}$ & -3.2523 & -3.305692 & 0.0000 & -3.2523 & -0.4096 & -3.305690 \\
\hline$g_{10}$ & -2.1880 & -2.227595 & 0.0000 & -2.1880 & -0.3046 & -2.227594 \\
\hline$g_{11}$ & -0.8922 & -0.912074 & 0.0000 & -0.8922 & -0.1537 & -0.912073 \\
\hline$g_{12}$ & 0.3600 & 0.362216 & 0.0000 & 0.3600 & 0.0153 & 0.362216 \\
\hline$g_{13}$ & 1.4245 & 1.447443 & 0.0000 & 1.4245 & 0.1754 & 1.447442 \\
\hline$g_{14}$ & 2.2253 & 2.264927 & 0.0000 & 2.2253 & 0.3046 & 2.264926 \\
\hline$g_{15}$ & 2.7205 & 2.770727 & 0.0000 & 2.7205 & 0.3879 & 2.770725 \\
\hline$g_{16}$ & 2.8878 & 2.941753 & 0.0000 & 2.8878 & 0.4166 & 2.941751 \\
\hline
\end{tabular}




\section{CURRICULUM VITAE}

Nevzat G. Gencer was born in Istanbul, Turkey in 1963. He received the B.S. degree in Electrical and Electronics Engineering from the Bosphorus University, in 1985. He entered the M.S. program in Mathematical Department of Middle East Technical University (METU) and completed the conditional undergraduate courses on Applied Mathematics in 1986. In 1987, he entered the M.S. program in Electrical and Electronics Engineering Department of METU, receiving the M.S. degree in 1988. He is currently working towards the $\mathrm{Ph} \mathrm{D}$. degree in the same department.

In 1987, he joined the Electrical and Electronics Engineering Department of METU as a research assistant. Since 1992 Spring Semester, he has been working as an instructor in the the same department. His areas of interest are Electrical Impedance Tomography, Neuromagnetic Imaging and Signal Processing.

Nevzat G. Gencer got the Erol Gelenbe One of the Best M.S. Thesis award in 1988.

\section{PUBLICATION LIST}

Papers

[24], [30], [44], [45] in the REFERENCES list.

Conference Papers

[19]-[23],[25]-[29], [41]-[43], [55] in the REFEENCES list 